\title{
Elucidating the role of LRRK2 kinase activity in the innate immune system
}

\author{
by
}

\section{Michaela Lunn}

A thesis submitted to the Faculty of Graduate and Post-Doctoral Affairs in partial fulfillment of the requirements for the degree of:

Master of Science

Neuroscience

\author{
Carleton University \\ Ottawa, Ontario
}

(C) 2019

Michaela Lunn 


\section{Abstract}

Mutations in LRRK2 are linked to three distinct diseases: Parkinson's disease (PD), Crohn's disease, and leprosy. The main pathogenic variant of LRRK2 associated with Parkinson's disease is the p.G2019S mutant, which causes increased kinase activity of the protein. Recently, a role for LRRK2 has been implicated in the immune system; however, the exact contribution of the kinase activity in this function remains unknown. We have used mice with a Lrrk2 knock-in p.D1994S mutation, which renders the protein kinase-dead, and three distinct infection paradigms to investigate this role: a systemic, nasal inoculation of reovirus serotype-3 Dearing (T3D); an intracerebral, direct-brain infection of reovirus T3D (both cause encephalitis); and a systemic, peripheral infection by Salmonella typhimurium (causing sepsis). Lrrk2 kinase-dead mice have increased survival compared to wild-type mice following a systemic reovirus T3D infection, with a slight increase in viral titre in the lungs at the early stage of disease. Nevertheless, loss of Lrrk2 kinase activity had no effect on survival from sepsis or bacterial load following i.v. inoculation. Lastly, Lrrk2 kinase-dead mice had the same survival rate as wild-type mice following a direct-brain infection by reovirus T3D. We demonstrated that in the context of both systemic infection models (i.e., viral and bacterial) Lrrk2 kinase is not required for the host's immune response to control virulent pathogens (when compared with wild-type Lrrk2 expression) and may in fact be protective in certain paradigms (i.e., systemic reovirus infection leading to encephalitis). Additionally, we have shown that Lrrk2 seems to exert its immune function predominantly in the periphery rather than the brain, and that the p.G2019S mutation confers a gain-of-function. Taken together, these data will provide important insights into LRRK2 biology, PD pathogenesis, and cause-directed therapies for all three diseases affected by allelic variants at this locus. 


\section{Acknowledgements}

I owe a great deal of gratitude to many people that have helped myself and this thesis get to this point today. First and foremost, thank you to my two wonderful supervisors, Dr. Michael Schlossmacher and Dr. Shawn Hayley. Thank you both for encouraging me to succeed, pushing me to my full potential, and allowing me to grow as a scientist.

A very special thank you to additional equally important mentors: Dr. Julianna Tomlinson, Dr. Bojan Shutinoski, and Dr. Earl Brown. Julie, thank you for being an amazing mentor, giving me advice on anything I ask, and always encouraging me to my highest potential. Bojan, thank you for being an incredible teacher and always showing me patience and understanding. Earl, thank you for all the extra time you took to help me understand topics and to answer questions any day of the week.

To everyone at the Hayley lab, thank you for being welcoming and inclusive when I found myself at Carleton. To my dear friends at the Schlossmacher lab, thank you for making my time in (and outside of) the lab tremendously enjoyable and memorable. I will cherish our times together for years to come.

To my parents, Allan and Christine, my sister, Yvonne, and my roommate, Red, thank you for offering so much support, and for listening to all my woes, my triumphs, and my endless banter about LRRK2. You have kept me sane and smiling throughout these two years.

Lastly, but not least, I would like to thank the wonderful people at the Parkinson's Research Consortium for believing in me and this research and supporting me throughout this project. 


\section{Table of Contents}

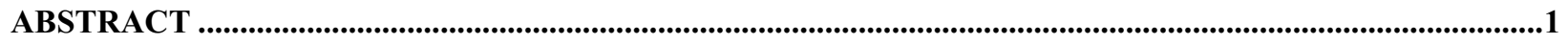

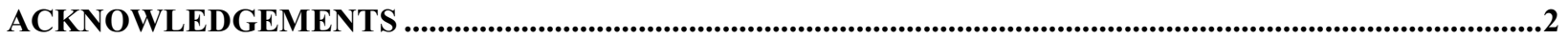

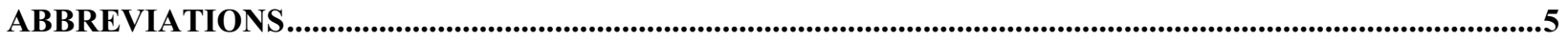

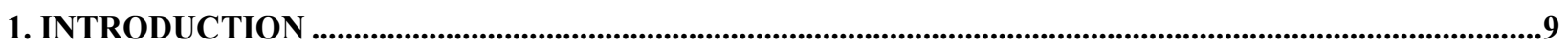

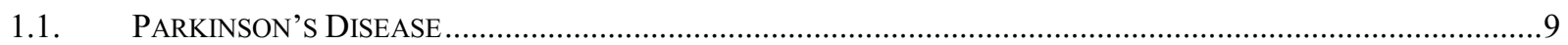

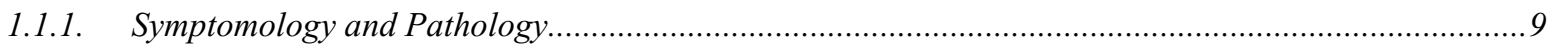

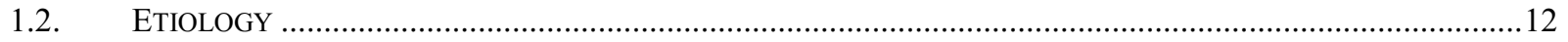

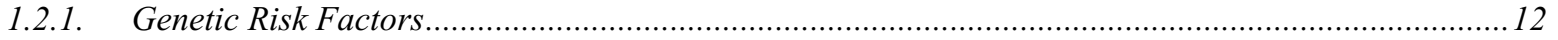

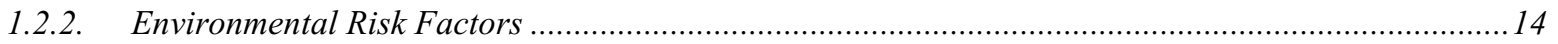

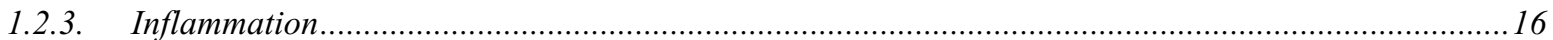

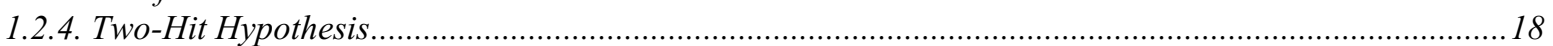

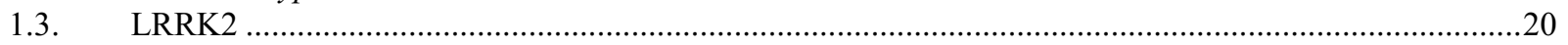

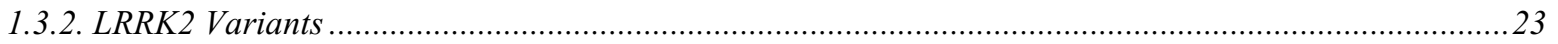

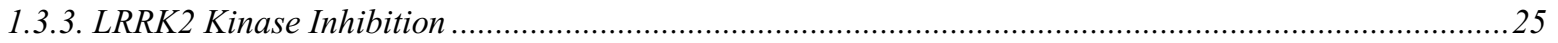

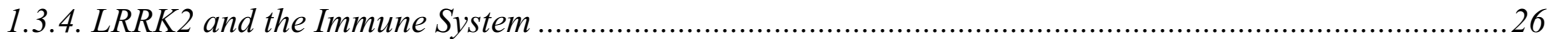

1.4. THE ROLE OF LRRK2 KINASE IN INNATE IMMUNE FUNCTION ...................................................................

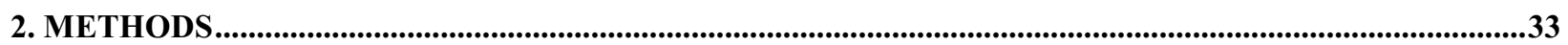

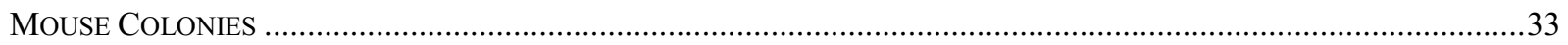

RESPIRATORY ENTERIC ORPHAN SEROTYPE 3 DEARING VIRUS …................................................................3

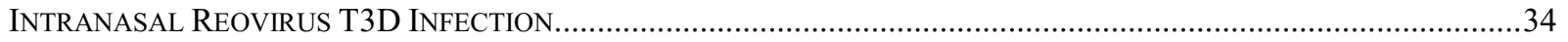

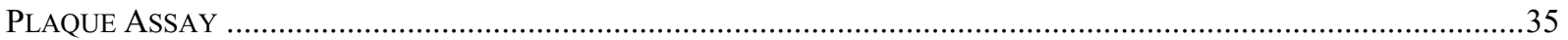

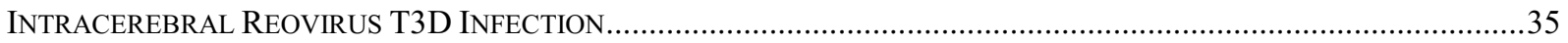

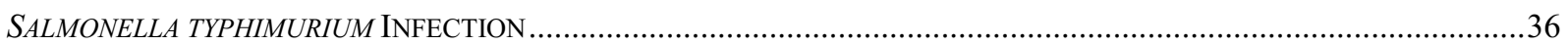

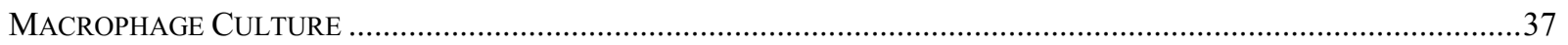

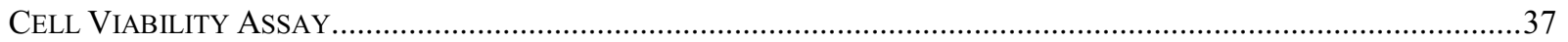

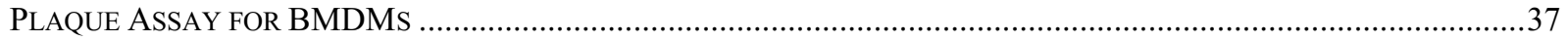

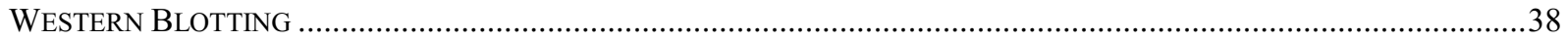

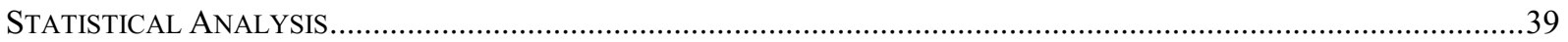

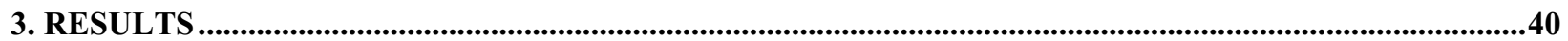

LRRK2 IS EXPRESSED IN WILD-TYPE MICE AS EARLY AS P1 IN THE BRAIN AND SPLEEN, AND LRRK2 EXPRESSION APPEARS

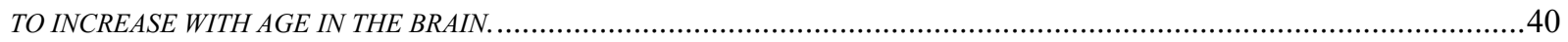

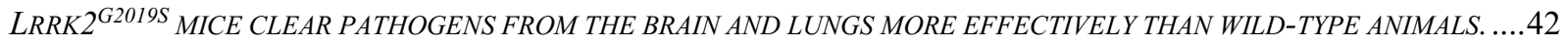

LRRK2 KINASE-DEAD HETEROZYGOUS MICE FOLLOW A SIMILAR SEX BIAS AS SEEN WITH OTHER LRRK2 MUTANTS. .........55 INTRACEREBRAL INOCULATION: LRRK2 KINASE-DEAD MICE HAVE AN EQUAL SURVIVAL RATE AS HETEROZYGOUS AND WILD-TYPE MICE FOLLOWING DIRECT INFECTION OF THE BRAIN WITH REOVIRUS T3D, BUT HAVE A DECREASED VIRAL TITRE IN THE BRAIN AT THE LATER STAGE OF DISEASE. ...................................................................................5

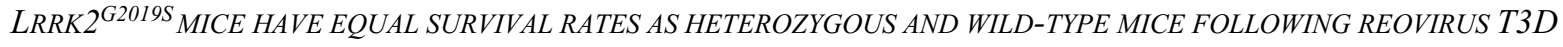

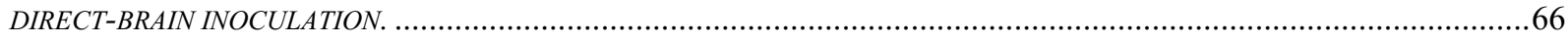
IN RESPONSE TO A SYSTEMIC BACTERIAL INFECTION, LRRK2 KINASE-DEAD MICE BEHAVE THE SAME AS WILD-TYPE MICE

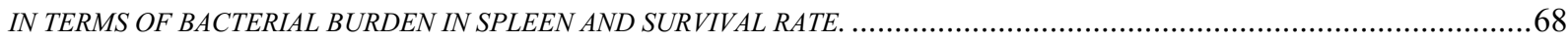
IN VITRO, LRRK2 KINASE-DEAD-DERIVED MACROPHAGES HAVE DECREASED IMMUNE SIGNALING AS INDICATED BY

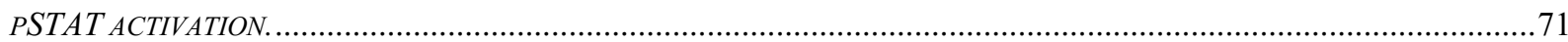
TREATMENT WITH A SELECTIVE LRRK2 KINASE INHIBITOR, MLI-2, IN BONE MARROW-DERIVED MACROPHAGES TO EXAMINE CELL VIABILITY AND DETERMINE VIRAL LOAD FOLLOWING INFECTION ............................................... 72

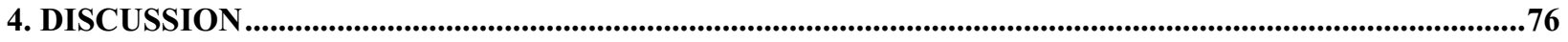

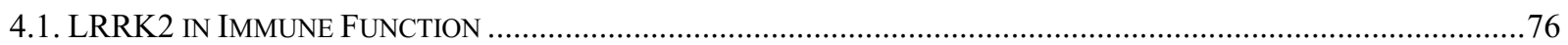

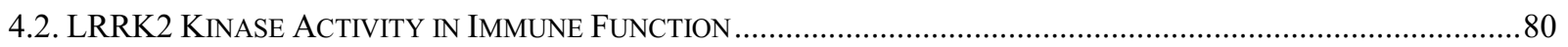

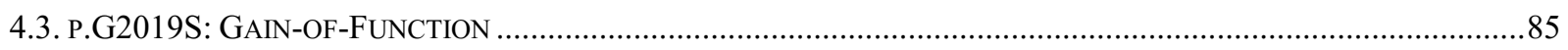


4.4. LRRK2 ACTS IN A PATHOGEN- AND ORGAN- DEPENDENT MANNER........................................................ 86

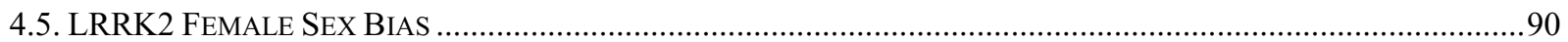

4.6. LRRK2 IMMUNE FUNCTION: BRAIN VS. PERIPHERY ….......................................................................... 92

5. CONCLUSION ..............................................................................................................................................97

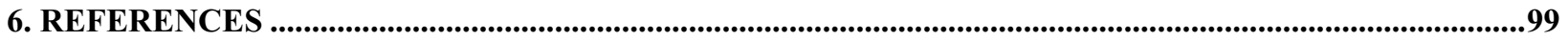




\begin{tabular}{|c|c|}
\hline \multicolumn{2}{|c|}{ Abbreviations } \\
\hline ANOVA & Analysis of variance \\
\hline BMDM & Bone marrow-derived macrophage \\
\hline $\mathrm{CD}$ & Crohn's disease \\
\hline $\mathrm{CFU}$ & Colony forming unit \\
\hline CNS & Central Nervous System \\
\hline DA & Dopamine \\
\hline DBS & Deep brain stimulation \\
\hline DPI & Days post infection \\
\hline dsRNA & Double-stranded RNA \\
\hline Gba1 & Glucocerebrosidase 1 \\
\hline GDI & Guanosine nucleotide dissociation inhibitor \\
\hline $\mathrm{H} \& \mathrm{E}$ & Hematoxylin and eosin \\
\hline IFN & Interferon \\
\hline IHC & Immunohistochemistry \\
\hline IL & Interleukin \\
\hline IP & Intraperitoneal \\
\hline $\mathrm{KO}$ & Knockout \\
\hline LB & Lewy Body \\
\hline $\mathrm{LD}_{50}$ & Lethal dose 50 \\
\hline LPS & Lipopolysaccharide \\
\hline LRR & Leucine rich repeat \\
\hline LRRK2 & Leucine rich repeat kinase 2 \\
\hline MAO-B & Monoamine oxidase B \\
\hline
\end{tabular}




$\begin{array}{ll}\text { MAPKKK } & \text { Mitogen-Activated Protein Kinase Kinase Kinase } \\ \text { M-CSF } & \text { Macrophage colony-stimulating factor } \\ \text { MOI } & \text { Multiplicity of infection } \\ \text { MPP+ } & \text { 1-methyl-4-phenylpyridinium } \\ \text { MPTP } & \text { 1-methyl-4-phenyl-1,2,3,6-tetrahydropyridine } \\ \text { mtDNA } & \text { Mitochondrial DNA } \\ \text { PBS } & \text { Phosphate-buffered saline } \\ \text { PD } & \text { Parkinson's Disease } \\ \text { PFU } & \text { Plaque forming unit } \\ \text { Poly I:C } & \text { Polyinosinic:polycytidylic acid } \\ \text { pSTAT } & \text { Phosphorylated STAT } \\ \text { REM } & \text { Rapid eye movement } \\ \text { ROS } & \text { Reactive oxygen species } \\ \text { SEM } & \text { Standard error of the mean } \\ \text { SNpc } & \text { Substantia Nigra Pars Compacta } \\ \text { T3D } & \text { Type 3 Dearing } \\ \text { TNF } & \text { Tumor necrosis factor } \\ \text { TLR } & \text { Toll-like receptor } \\ \text { tSTAT } & \text { Total STAT } \\ \text { VSV } & \text { Vesicular stomatitis virus } \\ \text { VTA } & \text { Ventral tegmental area } \\ \text { WT } & \text { Wild-type } \\ \end{array}$




\section{List of Figures}

Fig. 1.1 The structure of LRRK2 protein and identified mutations.

Fig. 1.2 Lrrk2 knockout (KO) animals have decreased survival and show no change in viral burden compared to wild-type mice in response to a viral infection.

Fig. 1.3 Lrrk2 ${ }^{\mathrm{G} 2019 \mathrm{~S}}$ female mice have decreased survival in response to a viral infection compared to wild-type mice.

Fig. 1.4 Lrrk2 ${ }^{\mathrm{G} 2019 \mathrm{~S}}$ mice are protected against a Salmonella typhimurium infection, whereas lack of Lrrk2 protein causes increased bacterial burden in the spleen.

Fig. 2.1 Schematic representation of the experimental paradigms employed in this thesis.

Fig. 3.1 Lrrk2 is present in wild-type spleens as early as p1, and its detection increases in the brain with age.

Fig. 3.2 Lrrk $2^{\mathrm{G} 2019 \mathrm{~S}}$ mice have lower viral titres in the brain and lungs following reovirusT3D nasal infection compared to wild-type mice.

Fig. 3.3 Lrrk2 ${ }^{\text {D1994S }}$ mutant mice have equal Lrrk2 protein expression but no kinase activity in brain and lung compared to wild-type mice following reovirus infection.

Fig. 3.4 Lrrk2 $2^{\text {D1994S }}$ mutant mice show improved survival from encephalitis with a female sex bias compared to wild-type mice.

Fig. 3.5 Lrrk $2^{\mathrm{D} 1994 \mathrm{~S}}$ mutant mice have increased viral burden in the lungs early in disease but show no change in viral burden at the late stage disease following a viral, nasal infection compared to wild-type mice.

Fig. 3.6 The increased viral burden in the lungs early in disease following a viral, nasal infection in Lrrk2 ${ }^{\text {D1994S }}$ mutant mice follows a female sex bias.

Fig. 3.7 Lrrk $2^{\mathrm{WT} / \mathrm{DS}}$ heterozygous mice show a sex bias as previously seen with other Lrrk2 variants.

Fig. 3.8 Loss of Lrrk2 kinase activity does not affect survival following an intracerebral injection of reovirus T3D.

Fig. 3.9 Loss of Lrrk2 kinase activity causes an unexpected decrease in viral titres with a female sex bias, following an intracerebral injection of reovirus T3D.

Fig. 3.10 Loss of Lrrk2 kinase activity does not affect viral titres at 3 days post infection following an intracerebral viral injection compared to wild-type mice. 
Fig. 3.11 Lrrk2 $2^{\mathrm{G} 2019 \mathrm{~S}}$ mice have equal survival rates as heterozygous and wild-type mice following an intracerebral reovirus T3D inoculation.

Fig. 3.12 Loss of Lrrk2 kinase activity does not affect the survival or bacterial burden in murine spleens in response to Salmonella typhimurium infection.

Fig. 3.13 Genetically ablating Lrrk2 kinase activity causes a decrease in the pSTAT/tSTAT ratio, indicating a decrease in the activation of immune signalling.

Fig. 3.14 Pharmacologically inhibiting Lrrk2 kinase activity does not affect cell viability or viral burden in cells. 


\section{Introduction}

\subsection{Parkinson's Disease}

Parkinson's Disease (PD) is a complex, progressive neurodegenerative motor disorder that affects $1-3 \%$ of individuals 85 years of age and over. Although prevalence of PD increases with age, still up to 4\% of PD patients are diagnosed before the age of 50 (reviewed in Kang and Marto, 2017). Parkinson's disease was first identified by Dr. James Parkinson in 1817 when he observed what he termed 'shaking palsy' in multiple patients. It consisted of a resting tremor, along with other non-motor symptoms such as sleep abnormalities and constipation (Parkinson, 2002). Since the discovery of this disease centuries ago, and despite great research efforts in the field of PD and neurodegeneration, the exact etiology has yet to be elucidated and a complete treatment or cure have yet to be identified.

\subsubsection{Symptomology and Pathology}

The clinical hallmarks of PD are identified as tremor, rigidity, postural instability, and bradykinesia (reviewed in Tysnes and Storstein, 2017). However, PD is much more than motor deficits: dementia occurs in up to $83 \%$ of late-stage PD patients and there are many non-motor symptoms that appear in the prodromal phase and continue for the entire course of disease (Kalia and Lang, 2015). Constipation, sleep disturbances, and loss of olfactory function are the first symptoms to appear in PD patients and can occur as early as 20 years before diagnosis, during the prodromal phase. In fact, olfactory dysfunction (hyposmia) is seen in $90 \%$ of idiopathic PD cases, and some familial cases of PD (specifically seen in PARK8 genetic cases) (Doty, 1988). Additionally, anxiety and depression are common co-morbidities of PD and occur in at least 50\% 
of PD cases. The exact neurobiology between these non-motor symptoms and PD remain complex (reviewed in Ferrer, 2011).

Pathologically, PD is identified by the loss of dopaminergic neurons in the Substania nigra pars compacta $(\mathrm{SNpc})$. These dopaminergic neurons mainly innervate the striatum, and this dopamine deficiency within the basal ganglia via the direct and indirect pathway leads to the movement dysfunctions seen in PD patients (Kalia and Lang, 2015). By the time of diagnosis, up to $50 \%$ of the dopaminergic neurons in the SNpc are lost, and up to $80 \%$ of dopamine release in the striatum is lost (Bohnen et al., 2006; Greffard et al., 2006).

Another pathological manifestation of 'typical PD' includes the formation of Lewy bodies (LB), which in PD is the accumulation of misfolded $\alpha$-synuclein protein, which can be found in neurons in the brain, and also in the spinal cord, vagus nerve, and adrenal medulla. There are also small amounts of tau, ubiquitin, and other proteins found in these LB (Goedert et al., 2013). Because of the accumulation of $\alpha$-synuclein, PD is termed a synucleinopathy. In neurons, LB can be found in the soma and dendrites (termed Lewy neurites) (Kalia and Lang, 2015). It has yet to be identified what is causing the accumulation of misfolded $\alpha$-synuclein in PD, and furthermore if these LB are disease-causing, protective, or mere bystanders of disease.

Despite the loss of dopamine-producing cells in the SNpc and the presence of $\alpha$-synuclein pathology in the brain, PD is not nigrostriatal-, dopamine-, or $\alpha$-synuclein-specific in every case. As mentioned, many symptoms of PD are non-motor and occur outside of the CNS. Additionally, some familial forms of PD do not have Lewy body formation (i.e., autosomal recessive PD due to Parkin deficiency) (Healy et al., 2004). As such, PD is known as a very heterogeneous disease, both symptomatically and pathologically. 


\subsubsection{Treatment}

As of 2019, there is no cure for PD. The gold-standard treatment for PD is currently dopamine-replacement via pharmacological levodopa treatment (LeWitt, 2008). Levodopa is the precursor for dopamine and can cross the blood brain barrier (which dopamine cannot). There it is converted into dopamine, enhancing the intracerebral dopamine concentration. However, levodopa is not effective for all PD patients and even so, over time, concentrations may need to be increased or its effects may begin to diminish in patients where it was once useful (Kalia and Lang, 2015). Additionally, levodopa often has adverse side effects, such as nausea, dizziness, and somnolence. Levodopa can also commonly cause dyskinesia in patients, leading to hyperkinetic movements, chorea, and dystonia (LeWitt, 2008). Other pharmacological treatments of PD include dopamine agonists, MAO-B inhibitors, and anti-cholinergics (Baba et al., 2002; Chung et al., 2015).

An established treatment for advanced motor signs in PD is deep brain stimulation (DBS), where the subthalamic nucleus or globus pallidus internus is stimulated to treat the motor symptoms of PD by normalizing the neuronal firings in this region (Rodriguez-Oroz et al., 2005). This has been found to be effective in some moderate-to-severe PD patients, although it is an invasive treatment and is not a cure, as neurodegeneration continues to progress (Kalia and Lang, 2015). Secondly, DBS is costly and not effective on all patients. It also can result in cognitive side effects in some patients, including increasing the severity of dementia (Rodriguez-Oroz et al., 2005).

The present pharmacological and surgical treatments of PD aim to temporarily treat the symptoms of disease and are not useful lifelong treatments, as the beneficial effects of both tend to diminish with time. Many PD patients are on a cocktail of medications in order to treat each symptom individually. There has been substantial research done to establish a cause-directed 
treatment for PD. For example, means to replace the dopaminergic cell loss in PD, using cell-based approaches such as stem-cell derived neurons to replace lost dopaminergic neurons in PD patients, or treatments to target $\alpha$-synuclein accumulation, such as immunotherapies to increase clearance (reviewed in Stoker et al., 2018). In later sections, the production of LRRK2 kinase inhibitors as a treatment for PD will be discussed.

\subsection{Etiology}

There are genetically two forms of Parkinson's disease: idiopathic (or sporadic) and familial (or genetic). Idiopathic accounts for about $\geq 90 \%$ of cases whereas only $\leq 10 \%$ of cases have a clear genetic link (reviewed in Kang and Marto, 2017). The exact etiology of Parkinson's disease is unknown; however, multiple genetic and environmental risk factors have been identified which seem to contribute to both idiopathic and familial PD. Both the genetic and environmental risk factors are of utmost importance in this thesis.

\subsubsection{Genetic Risk Factors}

There have been over 20 susceptibility loci identified for PD since the first gene was identified in 1997, $S N C A$, which encodes $\alpha$-synuclein and is inherited in an autosomal dominant manner (Munoz et al., 1997). As mentioned, $\alpha$-synuclein misfolds and aggregates in PD, one of the hallmarks of disease. SNCA mutations are very rare, and appear as point mutations, duplication, or triplication events of the gene, where triplications lead to a more severe and progressive form of the disease than duplication cases (Chartier-Harlin et al., 2007). 
The most common recessive mutation leading to early onset PD is caused by Parkin mutations. Parkin is an E3 ligase and much research has been conducted linking Parkin to mitochondrial dynamics, reactive oxygen species (ROS) production, and neuron degeneration (Kitada et al., 1998; Xiao et al., 2017). Interestingly, patients with Parkin-derived PD rarely have Lewy body pathology (Johansen et al., 2018). Mutations in PINK1 and DJ-1 are also associated with recessive PD, impaired mitochondrial function, and ROS dysregulation (Valente et al., 2004; Bonifati et al., 2003).

The most common genetic risk factor associated with PD is the gene encoding acid- $\beta$ glucocerebrosidase 1: Gbal (Nalls et al., 2014). Gbal was identified through epidemiological studies examining individuals with Gaucher's disease (Bembi et al., 2003). It is thought that in Gaucher's disease, glucocerebrosidase is defective and cannot perform its catalytic functions, leading to lipid substrate accumulation (Brady et al., 1965). It was found that the relatives of Gaucher's disease patients were much more likely to develop PD. It has now been identified that carrying one or two mutant Gbal alleles greatly increases the risk for PD (Anheim, et al. 2012). Gbal-associated PD causes a form of disease that has a more severe progression and a greater cognitive decline (Brockmann et al., 2011). It is reported that Gbal carriers have as high as 20-30 fold higher risk of developing PD than the general public (Sidransky et al., 2009).

The second most common autosomal dominant form of PD are mutations found in the LRRK2 gene, of which seven mutations have been pathogenically linked to PD, all of which fall in the catalytic domains of the protein (Zimprich et al., 2004). There are numerous other mutations throughout the protein that also confer increased risk for PD (reviewed in Gasser, 2009). Mutations in $L R R K 2$ reportedly account for about $4 \%$ of familial cases of PD and $1-2 \%$ of sporadic (Healy 
et al., 2008). The most commonly found mutation in LRRK2 is the p.G2019S variant in the kinase domain, which causes a two- to three-fold increase in the phosphorylating activity of the protein (Kachergus et al., 2005). The p.G2019S mutation is an autosomal dominantly inherited mutation, but has a low penetrance to cause PD ( 28\%) (Healy et al., 2008). LRRK2 is the focus of this thesis and will be discussed at length in later sections.

\subsubsection{Environmental Risk Factors}

Although the identified genetic risk factors provide extensive insight into the etiology of disease in PD, the clear majority of PD cases have no clear genetic component. In addition to genetics, environmental exposure(s) is thought to be an important contributing factor, and several environmental risk factors have been identified. One of the first identified was exposure to neurotoxins, the most common example being MPTP, which is the pro-compound of the neurotoxin MPP+ (Langston et al., 1983). This was identified in a group of drug users who used MPTP recreationally, and developed parkinsonism. MPP+ is selectively taken up by dopaminergic neurons and blocks mitochondria via complex I (Sian et al., 1999) MPP+ parkinsonism causes selective degeneration of the dopamine-producing neurons in the SNpc, whereas dopaminergic neurons in the VTA are spared (Blanchard et al., 1994). Additionally, monkeys treated with MPTP to induce parkinsonism were responsive to L-dopa treatment (Burns et al., 1984). This demonstrates a role for acute toxin exposure and a parkinsonian phenotype.

Pesticide exposure, including the herbicide paraquat and insecticide rotenone, has also been highly linked to conferring risk for PD (Schapira and Jenner, 2011). Systemic paraquat and rotenone treatment has led to PD-like behaviour and pathology in rodents that is comparable to 
MPTP-induced parkinsonism is rodents. Rotenone and paraquat are highly associated with PD risk in multiple human case-controls study, and both pesticides have mechanisms which disrupt mitochondrial function and oxidative stress regulation (reviewed in Bove and Perrier, 2011). Interestingly, previous research in the Hayley lab has identified that pre-treatment with a viral mimic (i.e., Poly I:C) or bacterial mimic (i.e., LPS) enhances the neurotoxic effects of paraquat, as seen by a great degeneration of dopaminergic neurons and more pronounced PD-like symptoms in rodents (Dwyer, Hayley, unpublished). It is proposed that this could be due to the sensitivity of the SNpc and the dopaminergic neurons within. The SNpc contains the most abundant amount of microglia in the brain, and it is possible that when primed by an infectious agent, the microglia become overstimulated and cause damage to the dopaminergic neurons in the SNpc (Skaper et al., 2018).

As of late, there is a growing interest in the field on the effects of microbial infection in PD. A bacterial mimic, lipopolysaccharide (LPS), which is a component of the bacterial cell wall, is often used in these studies. In many studies, it has been shown that infectious mimics such LPS can lead to parkinsonian behavior and death of dopaminergic cells in mouse models (Arai et. al., 2006). Next, one research group has used the highly infectious influenza virus H5N1 to infect wild-type mice, and was able to demonstrate that the virus progressed from the peripheral nervous system to the CNS, where it then induced PD-like pathological changes, as well as the activation of microglia, increased phosphorylation of aggregated $\alpha$-synuclein, and lastly, degeneration of dopaminergic neurons in the SNpc even after resolution of the virus (Jang et al., 2009).

Similarly, following the 1918 influenza pandemic caused by type A H1N1, there was a coinciding outbreak of encephalitis lethargica (EL) and postencephalitic parkinsonism (first 
described by Constantin van Economo), suggesting that the disease may have been associated with the infection (Jordon, 1927). Furthermore, individuals born during the 1918 outbreak are at 2-3 fold higher risk of developing PD than those prior to 1918 or after 1924 (Martyn, 1997). The risk of parkinsonian symptoms increases with the number of influenza attacks, which poses a strong link between influenza exposure and parkinsonian behavior (Martyn, 1997). There is research that disagrees with the hypothesis that the influenza and PD outbreak were related, as RNA recovered from the postencephalitic patients with parkinsonism was not positive for genomic information related to influenza virus (Lo et al., 2003); however, that does not discount the notion that the virus could have triggered the initiation and/or progression of illness before eventually being cleared from the system.

In addition to these, heavy metals, agricultural occupation, rural living, and well water drinking have been indicated as risk factors. Of note, some of these environmental risk factors could heighten the exposure to more strongly correlated risk factors, such as infectious agents or pesticide exposure. Importantly, many of these risk factors may occur over extended periods of time, causing cumulative damage which may eventually manifest as clinical presentation (Kalia and Lang, 2015).

\subsubsection{Inflammation}

Inflammation is a key component of the innate immune response and is the first attempt by the host to eliminate the initial source of injury by concentrating the body's defensive agents to the site of infection via chemokines and cytokines. Although acute inflammation is critical for an efficient immune response, chronic inflammation can be detrimental to the host (Skaper et al. 2018). 
Glial cells, particularly microglia, are the key contributors to the neuroinflammatory response. The brain is considered to be an immune-privileged site, with the blood brain barrier limiting the infiltration of peripheral leukocytes (though not completely, as will be seen later in this thesis). As such, microglia are the resident immune cells of the CNS (reviewed in Hickman et al., 2018). They act, in conjunction with astrocytes, another type of glial cell, to ultimately maintain the homeostasis of the CNS. Microglia recognize and eliminate pathogens, clear toxic cellular debris, and prune futile synapses. Microglia exist in two states: a quiescent state, in which the microglia are in surveillance mode monitoring for threats; and an activated form, in which the microglia are morphologically and functionally distinct and respond to perceived threats (Hickman et al., 2018).

Neurodegeneration is often associated with the morphologically and functionally active state of microglia. In fact, there is consistently found to be more microglia in the activated state over quiescent state in post mortem PD brains (Sanchez-Guajardo et al., 2013). Microglia activation was augmented in post mortem brains of humans exposed to MPTP and mouse models using the same neurotoxin (Imamura et al., 2003). There is debate whether this activation of microglia is causal or a result of dopaminergic cell death in PD. It is highly possible that microglia may be triggered or primed by an exogenous substance (i.e., infectious agent), which may lead to their activated state and ultimately contribute to the death of the vulnerable dopaminergic neurons in the SNpc.

Additionally, in relevance to PD, it has been shown that pathological $\alpha$-synuclein can stimulate microglia activation via the transcription factor $\mathrm{NF \kappa B}$ and through pro-inflammatory cytokines such as TNF- $\alpha$ and IL-1 $\beta$ (Zhang et. al. 2017). 
Neuroinflammation has been implicated in other neurodegenerative diseases, including multiple sclerosis and Alzheimer's disease. In fact, inflammation has widely been considered a cardinal element in the pathogenesis of many neurodegenerative diseases. Some hypotheses suggest that neuroinflammation may prime neural populations for subsequent neurodegeneration

(Chen et al., 2016). There is evidence of increased concentrations of pro-inflammatory cytokines in the brain that can be seen in early stages of neurodegenerative diseases, which is likely the result of activated microglia, and as will be discussed in this thesis, the recruitment of infiltrating myeloid cells (Deleidi and Isacson, 2012).

\subsubsection{Two-Hit Hypothesis}

The Braak staging hypothesis was proposed in 2003, and aims to explain the sequential onset of non-motor followed by motor symptoms of PD by staging the progression of Lewy inclusion pathology (Braak et al., 2003). In stage 1 and 2 of Braak staging, Lewy pathology are found in the lower brainstem (medulla and pons) and olfactory bulb, and this staging coincides with olfactory dysfunction and REM sleep disturbances in the prodromal (prior to motor) stages of disease, respectively. In stage 3 and 4, the Lewy pathology is seen in the SNpc, as well as other midbrain areas, which then coincides with the motor defects prominently seen in the typical, symptomatic stage of PD pathogenesis. Lastly, in stages 5 and 6, the Lewy pathology eventually reaches the cortex, which is associated with the cognitive deficits seen in PD (Braak et al., 2003). It is unknown whether or not this LB pathology is causing the symptoms associated with each stage; likely, it contributes in part to the effect of the disease on each specific brain region. This hypothesis is not without criticism, as not all PD patients demonstrate pathology and / or 
symptomatology in line with Braak's staging; however, as clearly demonstrated, PD is extremely heterogeneous and it is expected that not all cases could be explained by one hypothesis.

Braak also proposes a two-hit hypothesis of PD pathogenesis, explained initially in Hawkes et al. (2007). The authors go further to suggest that a neurotropic pathogen may enter the brain via two routes: the nasal route via the olfactory bulb, or by first invading the enteric plexus of the gut and travelling retrogradely via the vagus nerve to the brain. The gut and the nasal system are two of the main interfaces with the environment and therefore often the initial site to encounter foreign agents (Hawkes et al., 2007; Hawkes et al., 2009). This hypothesis agrees with their original notion that Lewy body pathology also originates in the olfactory bulb and the brain stem (i.e., at the medulla oblongata, the entry / exit site of the vagus nerve) (Braak et al., 2003; Hawkes et al., 2007; Hawkes et al., 2009).

Building upon Braak's hypothesis, this thesis was structured on an overarching theory: PD is a complex disease that is a combination of genetic susceptibility and environmental exposure (Schlossmacher et al., 2017). As mentioned, there are many environmental risk factors identified for PD and at least 20 susceptibility genes. The low penetrance rate of many of the associated genes could be explained by the theory that a second, environmental 'trigger' is needed for the onset of the disease. Therefore, in theory, PD is a complex disease, requiring a sort of 'dual-hit' to induce pathogenesis.

Therefore, it is conceivable that extended exposure to an environmental risk factor superimposed on genetic susceptibility may be required for the onset of PD. It may require a certain threshold of environmental exposure (i.e., pesticide, infectious agent, metal) to initiate the pathological effects of the disease gene variant. In accordance with this, some environmental triggers, such as neurotoxins (MPTP), may require a lower threshold to initiate disease, than other 
environmental triggers, such as infectious agents, which are ubiquitous in the environment and it is the extensive exposure overtime that can lead to disease pathology and progression.

In the following sections of this thesis, an interaction between a major susceptibility gene (LRRK2) and the environmental exposure of interest (infectious agents) will be explored.

\subsection{LRRK2}

The LRRK2 protein, first identified in 2004, is very large (2527 amino acids and 286kDa) with multiple domains (Kang and Marto, 2017). It contains multiple protein-protein interaction domains such as the LRR domain and WD40 domain and has two enzymatic domains - a GTPase domain (ROC-COR domain) and a kinase domain (Fig. 1.1). The GTPase domain hydrolyzes GTP to GDP, and the kinase domain is a serine/threonine MAPKKK. LRRK2 functions mainly as a homodimer and in addition to its kinase and GTPase properties, is thought to be an important scaffolding protein (Kang and Marto, 2017). 


\section{D1994S}

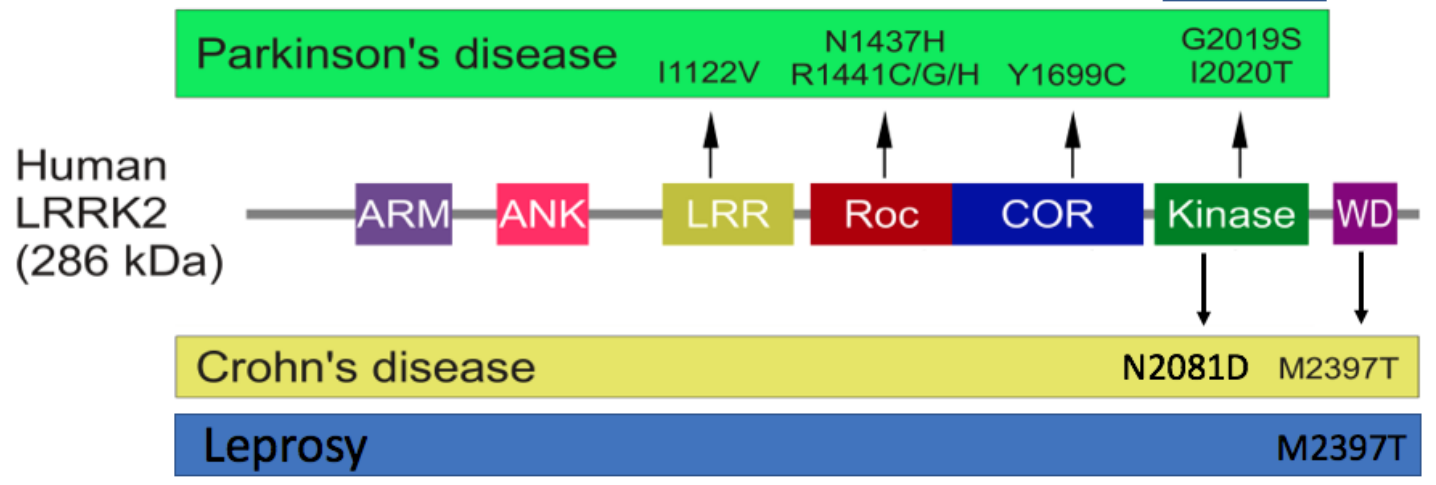

Fig. 1.1: The structure of LRRK2 protein and identified mutations, with specific mutations indicated including the most common PD-linked mutation p.G2019S; Crohn's disease- and leprosy-associated mutations; and the kinase-dead mutation, p.D1994S. ARM: armadillo domain; ANK: ankyrin repeat; LRR: leucine-rich repeat; Roc: Ras of complex; COR: C-terminal of Roc; WD: WD40. Adapted from Ho et al. (2014).

\subsubsection{LRRK2: Physiological Function}

Though the exact physiological role of LRRK2 is unknown, the protein has been implicated in many functions, including neurite outgrowth, microtubule dynamics, autophagy, and vesicle trafficking (Macleod et al., 2002; Kawaki et al., 2012; Kett et al., 2012; Herzig et al., 2011). Multiple substrates for LRRK2 kinase have also been indicated, primarily autophosphorylation and a subset of Rab proteins (Steger et al., 2016).

Much research has been focused on LRRK2's role in the brain. Macleod et al. (2006) have demonstrated that mutations in LRRK2, specifically p.G2019S and p.I2020T which both increase the kinase activity of the protein, lead to a reduction in neuron length and axonal branching, in vitro and in vivo in rodents (Macleod et al., 2006). This could be due to LRRK2's ability to 
phosphorylate tubulin-associated tau, causing it to dissociate from microtubules, affecting neurite outgrowth (Kawaki et al., 2012).

This association led to a proposed role for LRRK2 in cytoskeletal dynamics via microtubule associations. It has been demonstrated that LRRK2 oligomerizes and creates filaments in vitro which form on top of existing microtubules, dependent on its kinase (in a closed conformation, structurally) and WD40 domains (Kett et al., 2012). This building on microtubules blocks kinesin activity, which can be reversed by LRRK2 kinase inhibitors which causes the kinase domain of the protein to be in open conformation (Peterson et al., unpublished).

LRRK2 has been heavily implicated in autophagy and vesicular trafficking. Firstly, LRRK2 is highly localized to lysosomes and endosomes in the cell. Research with Lrrk2 knockout mice indicate that Lrrk2 may be a suppressor of autophagy, as these mice had increased autophagic markers (i.e., LC3-II) and increased accumulation of vesicles, specifically seen in the lungs and kidneys. Of note, Lrrk2 kinase-dead mice have vesicle accumulation in the kidneys but not the lungs (Herzig et al., 2011). However, there are varying results and it is not yet clear if LRRK2 functions as negative or positive regulator of autophagy, and whether it exerts its effects directly or indirectly (reviewed in Roosen and Cookson, 2012).

Many mutations in LRRK2 associated with PD cause constitutive kinase activity, which may disrupt normal, physiological signaling, and therefore the substrates of LRRK2 kinase are of great interest. LRRK2 has been identified in the MAPKKK pathway, and autophosphorylates on Ser-1292 (Sheng et al., 2012). Of note, LRRK2 does not autophosphorylate on Ser-935 but this has been highly recognized as a measure for LRRK2 phosphorylative activity (Dzamko et al., 2010). The autophosphorylative function of LRRK2 can be used as a biochemical readout for LRRK2 kinase activity (Kang and Marto, 2107). 
Using phosphoproteomics, Steger et al. (2016) have identified a novel set of Rab GTPases as substrates for LRRK2, most notably Rab10 on threonine 73. The residues in which LRRK2 phosphorylates are on the switch II domains of these Rab proteins, notably in the region where Rab proteins interact with regulatory proteins, such as GDIs, in turn affecting the compartmentalization from cytosol to membrane. Overactive LRRK2 (i.e., p.G2019S mutant) promotes Rab protein membrane compartmentalization and accumulation. This disrupts Rab protein homeostasis, in turn disrupting intracellular trafficking (Steger et al., 2016). LRRK2 has since been shown to interact with Rab 8 and 10 to stimulate lysosome production and secretion following stress induction in vitro (Eguchi et al., 2018). This data is extremely important in indicating bona fide substrates for LRRK2 as well as further implicating it in vesicular trafficking.

\subsubsection{LRRK2 Variants}

The majority of familial PD-linked genes cause a monogenic PD that is unique from idiopathic PD. For example, the mutations in the Parkin protein cause early onset PD, or other mutations that are pathophysiologically unique from idiopathic PD, such as mutations that lack Lewy body pathology (Schulte and Gasser, 2011). In contrast, LRRK2-associated familial PD is, for the most part, clinically and pathologically indistinguishable from idiopathic PD (Kang and Marto, 2017). Additionally, mutations in LRRK2 are not only associated with familial PD, but also have been identified to confer risk for idiopathic PD. Therefore, studying the LRRK2 protein and LRRK2-associated PD will give great insight into not only familial PD, but also idiopathic PD and will hopefully give rise to therapeutics for both types of the disease.

There are over 50 identified variants of LRRK2, though not all pathogenic and not all are associated with PD (Klein and Schlossmacher, 2007). Seven variants have been pathogenically- 
linked to PD, with more identified to increase the risk of disease (Gasser, 2009). As mentioned, the p.G2019S mutation falls in the kinase domain of the protein and causes about two-fold increased constitutive kinase activity. Two neighboring mutations also fall in the kinase domain, the p.I2020T and p.I2012T mutations. The p.G2019S mutation is much more common than any of the other variants. Multiple mutations also fall in the ROC-COR domain (GTPase catalytic domain): p.R1441C/G/H and p.Y1699C (Shu et al., 2019). Interestingly, mutations in the GTPase domain also lead to an approximate four-fold increase in its kinase activity (West et al., 2007). Notably, there is incomplete penetrance associated with these mutations, with the most common variant, p.G2019S, having as low as $28 \%$ penetrance. Reports indicate that the penetrance rate may rise as high as $75 \%$ by age 80 (Healy et al., 2008).

Multiple mutations have been identified to confer risk for PD, some of which may be segregated to specific geographic areas. This includes the mutation p.G2385R which interestingly falls in the non-catalytic WD40 domain of the protein (Kett et al., 2012). There is also the GTPase mutation p.R1628P (Yu et al., 2009).

A striking new discovery has noted that the p.N2081D mutation in the kinase domain of LRRK2 confers risk for both Parkinson's and Crohn's disease (CD) (Hui et al., 2018). This identifies a second variant that has been associated with CD. The variant p.M2397T in the kinase domain of LRRK2 is pathogenically linked to both CD and leprosy (Hui et al., 2018; Fava et al., 2016). PD and CD also share variants in LRRK2 that confer increased protection against the disease: p.N551K and p.R1398H. It is shown that p.R1398H causes the GTPase domain to be constitutively active, whereas the p.N551K is proposed to affect the dimerization of the protein 
(Hui et al., 2018). Notably, in the sample set of Hui et al. (2017), all human carriers of p.N551K also carried the p.R1398H mutation, possibly indicating that both are required to confer protection.

\subsubsection{LRRK2 Kinase Inhibition}

As these mutations in LRRK2 cause increased kinase activity of the protein, LRRK2 kinase inhibitors have been created to be used as a therapeutic for PD (Alessi and Sammler, 2018), and are now entering phase I of clinical trials (DNL151) (Denali Therapeutics Inc., 2018). Theses inhibitors are ATP-competitive active-site kinase inhibitors (West, 2017). There are multiple inhibitors created such as MLi-2, LRRK2-IN-1, GSK2578215A, and HG-10-102-01. Some of the original LRRK2 kinase inhibitors had many off-target effects; however, more recent inhibitors such as MLi-2 show greater selectivity (Alessi and Sammler, 2018). These kinase inhibitors can either lock the kinase domain in an open conformation or a closed conformation, both ultimately inactivating the kinase activity of the protein, but can have differential effects on the functions of the protein, for example microtubule binding (Peterson et al., unpublished).

Of note, Herzig et al. (2011) have characterized a mouse model with a Lrrk2 p.D1994S knock-in mutation in the kinase domain of the protein, rendering the protein kinase-dead. They have noted that ablating the kinase activity causes the protein to be unstable, and mice with this mutation have about half the abundance of Lrrk2 as wild-type mice. Additionally, this genetic ablation of Lrrk2 kinase, as well as using a pharmacological inhibitor, has led to some kidney pathology, including vesicle accumulation (Herzig et al., 2011). Fuji et al. (2015) also identified that when using two separate LRRK2 kinase inhibitors in non-human primates, there was an increase in abnormal cytoplasmic accumulation of secretory lamellar bodies in type II 
pneumocytes, though this was not seen in the kidney. It is possible that inhibiting LRRK2 kinase could be harmful in some instances, and the kinase activity of LRRK2 must be fully elucidated to validate the safety of these inhibitors in humans.

\subsubsection{LRRK2 and the Immune System}

A novel function for LRRK2 has been identified as a component of the immune system (Hakimi et al., 2011; Shutinoski et al., 2019). To this point, LRRK2 has mainly been studied in the context of the brain. The new evidence of LRRK2 in the immune system must be considered in the context of safety of kinase inhibitors. It is possible that by inhibiting LRRK2 kinase activity in patients, it will put these individuals at greater risk to infection. In an elderly, immunosuppressed population, these infections could be extremely serious.

There is a multitude of evidence demonstrating LRRK2 in the immune system, particularly the innate immune system (Hakimi et al., 2011). Firstly, not only is LRRK2 much more highly expressed in the periphery (i.e., lung and kidney) than the brain, but LRRK2 is much more highly expressed in cells of the immune system (particularly neutrophils, monocytes, and macrophages) than neurons. Of note, LRRK2 is also more highly expressed in cells such as neutrophils and monocytes than T- or B-cells, suggesting a primary role for LRRK2 in the innate immune system rather than the adaptive immune system (Hakimi et al., 2011; Shutinoski et al., 2019). Next, LRRK2 can be upregulated by pathogens and infectious agents, as well as inflammatory signals such as IFN- $\gamma$ and NF- $\mathrm{kB}$, suggesting that LRRK2 is involved in pathogen response pathways and inflammation (Gardet et al., 2010; Hakim et al., 2011).

Lastly, mutations in LRRK2 have been linked to two other disease: Crohn's disease and leprosy (Hui et al., 2018; Fava et al., 2016). Both diseases do not occur in the brain and neither 
lead to neurodegeneration. It is puzzling therefore, as to why these three very different diseases (i.e., $\mathrm{PD}, \mathrm{CD}$, and leprosy) are all linked to mutations in the same gene. $\mathrm{CD}$ and leprosy are both characterized by inflammation and both require exposure to xenobiotic pathogens to manifest. There is growing evidence of the involvement of inflammation in PD and it is not impossible to conceive, considering the growing role for LRRK2 in the immune system, that there may be an effect of xenobiotic pathogens in the onset of PD.

Within the neuroscience field, our lab has conducted the first research into the role of Lrrk2 in the immune system (Hakimi et al., 2011). Our lab has found that when measuring survival, mice lacking the Lrrk2 protein showed significantly decreased survival in response to a systemic, viral infection, with heterozygous ( $\operatorname{Lrrk} 2^{\mathrm{WT} / \mathrm{KO}}$ ) mice being intermediate between WT and homozygous Lrrk2- knockout mice, suggesting a gene dosage effect (Fig. 1.2A). When measuring viral titre, there was no discernable difference between Lrrk2-knockout and wild-type mice in plaque forming units (PFU) (Fig. 1.2B) (Shutinoski et al., 2019). 

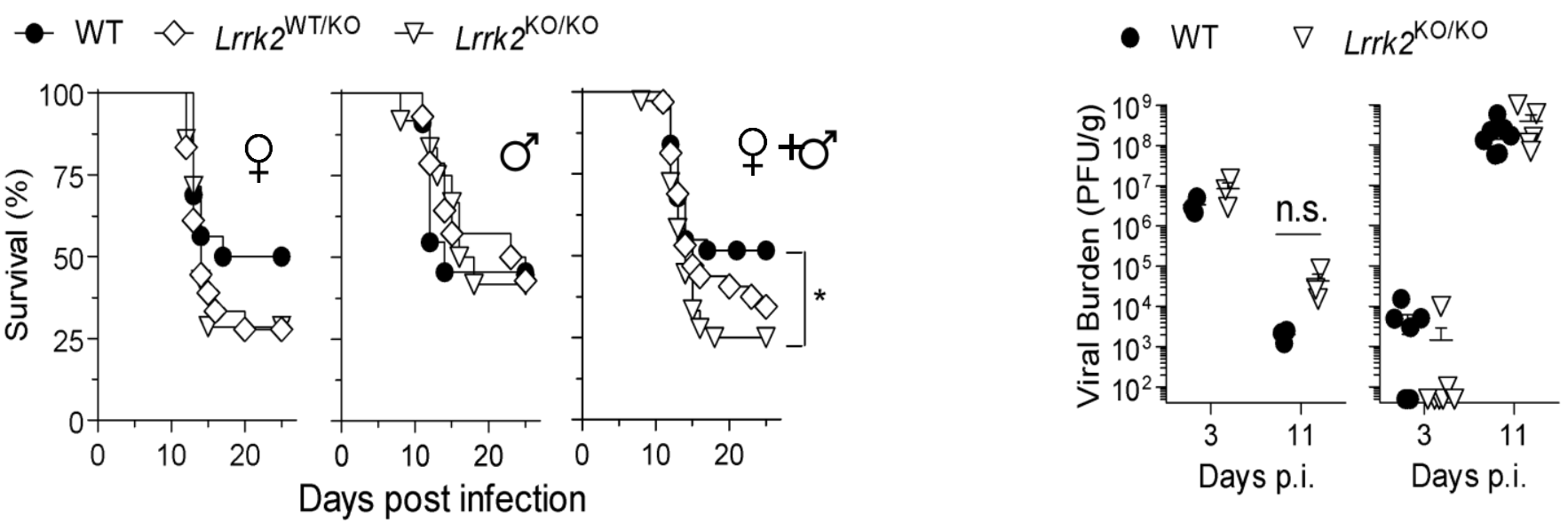

Fig. 1.2: Lrrk2 knockout (KO) animals have decreased survival and no change in viral burden compared to wild-type mice in response to a viral infection. Newborn mice were nasally inoculated with one $\mathrm{LD}_{50}$ of reovirus-T3D, leading to infection. (A) Lrrk2 knockout and wild-type mice were measured for survival following infection, and separated based on sex. Data were analyzed using the log-rank (Mantel-Cox) test. (B) Lrrk2 knockout (Lrrk2 ${ }^{\mathrm{KO} / \mathrm{KO}}$ ) and wildtype mice were sacrificed at 3dpi and 11dpi and lungs and brain were collected. Data are represented in PFU/g, where each symbol represents one animal. Data were statistically analyzed using the Mann-Whitney test. Error bars represent mean \pm SEM (data were generated by Mansoureh Hakimi, Ph.D., and published in Shutinoski et al., 2019). 
Because my colleagues had found differences in response to viral infection in Lrrk2 knockout mice, they next sought to find the effects of the PD-associated mutation p.G2019S in the same paradigm. When looking at the sexes pooled, or just males, there was no difference in survival against reovirus T3D infection compared to wild-type mice. Surprisingly, when only looking at females, Lrrk $2^{\mathrm{G} 2019 \mathrm{~S}}$ mice were significantly worse off and had a much-lowered survival compared to female wild-type littermates (Fig. 1.3) (data were generated by Mansoureh Hakimi, Ph.D., and published in Shutinoski et al., 2019).

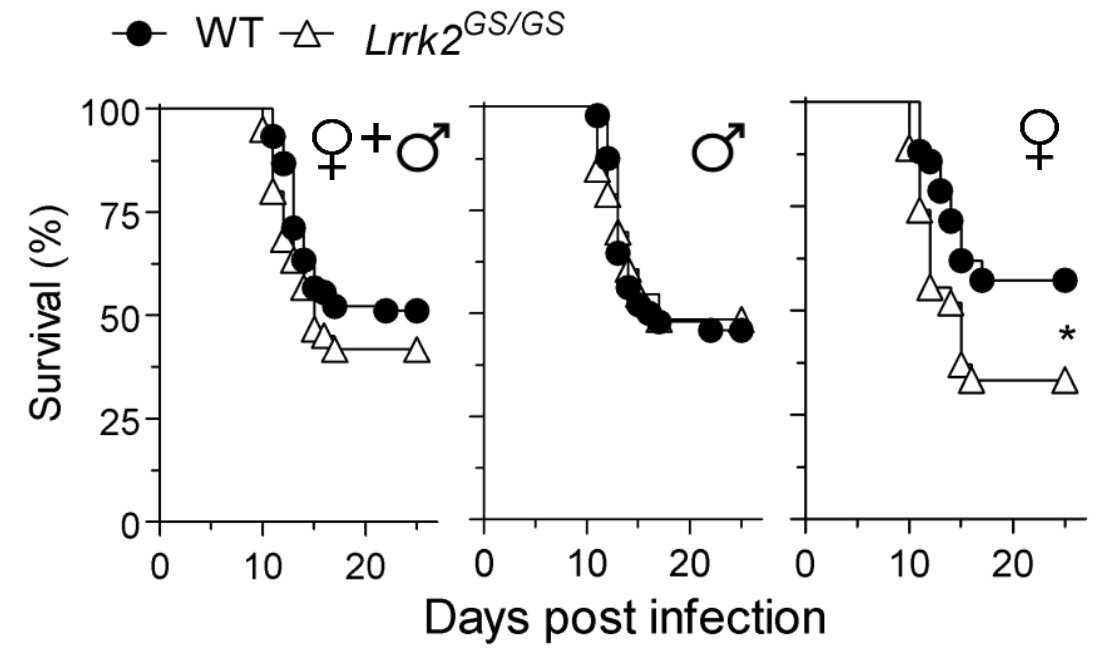

Fig. 1.3: Lrrk2 ${ }^{\mathrm{G} 2019 \mathrm{~S}}$ female mice have decreased survival in response to a viral infection compared to wild-type mice. Newborn mice were nasally inoculated with one $\mathrm{LD}_{50}$ of reovirus$\mathrm{T} 3 \mathrm{D}$, leading to infection. Lrrk2 ${ }^{\mathrm{G} 2019 \mathrm{~S}}$ and wild-type mice were measured for survival following infection, and separated based on sex. Data were analyzed using the log-rank (Mantel-Cox) test. (Shutinoski et al., 2019). 
Next, they investigated the effects of a systemic, bacterial infection in adult mice. Lrrk2 $^{\mathrm{G} 2019 \mathrm{~S}}$ homozygous and heterozygous mice had a significantly longer time-to-death than wild-types littermates. When measuring colony forming units (CFU) in the spleen to measure bacterial titre, both male and female $\operatorname{Lrrk}^{\mathrm{G} 2019 \mathrm{~S}}$ mice had significantly lower bacterial titres. Therefore, Lrrk2 ${ }^{\mathrm{G} 2019 \mathrm{~S}}$ mutant mice appear to have a heightened immune response in a bacterial paradigm, which corresponds to decreased bacterial titre and increased survival (Fig. 1.4A, B) (data were generated by Bojan Shutinoski, Ph.D. and published in Shutinoski et al., 2019).

In contrast, the absence of Lrrk2 (Lrrk2 KO) did not affect the response to infection in terms of survival; however, the absence of Lrrk2 caused a significant increase in the bacterial titre in the spleen compared to wild-types (Fig. 1.4C, D) (Shutinoski et al., 2019).

Taken together, these results indicated a role for LRRK2 in host immune responses, which seemed to be heightened by increased kinase activity (via a common, pathogenic mutant). Our laboratory's research question then became: Is the kinase function required for its immune function? 


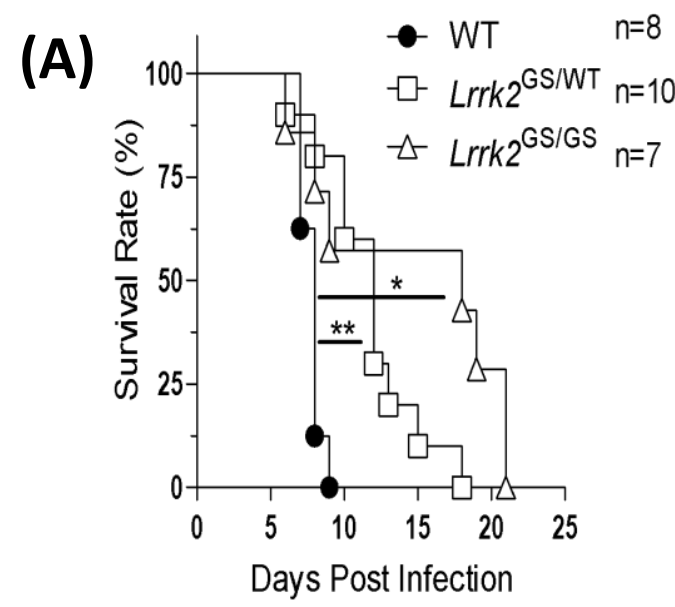

(C)

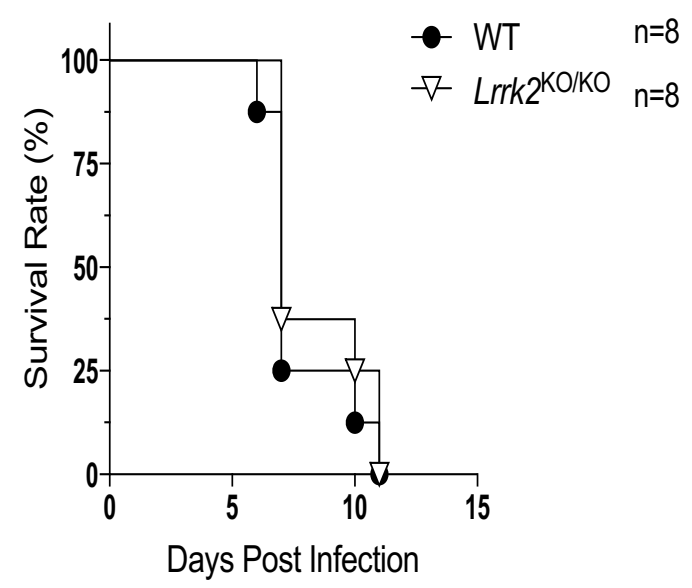

(B)

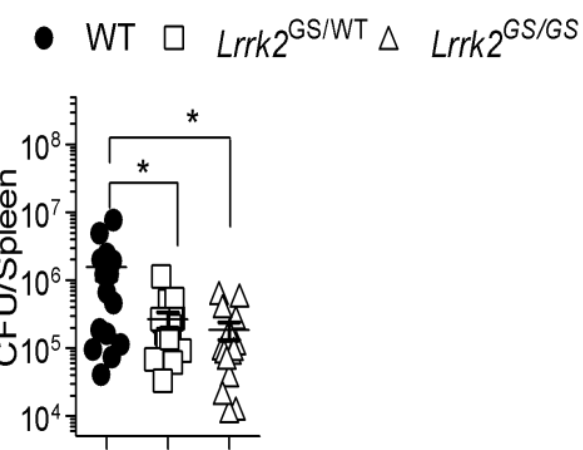

(D)

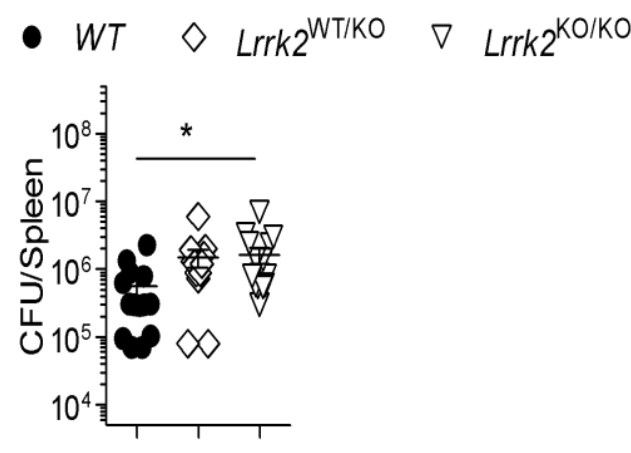

Fig. 1.4: Lrrk2 ${ }^{\mathrm{G2019S}}$ mice are protected against a Salmonella typhimurium infection, whereas lack of Lrrk2 protein caused increased bacterial burden in the spleen. Adult mice were infected intravenously with 200 CFU of Salmonella typhimurium. Survival rate (time-todeath) was measured in Lrrk2 ${ }^{\mathrm{G} 2019 \mathrm{~S}}$ (A) and Lrrk2 KO cohorts (C). CFU (Colony forming units) counts of Salmonella infected spleen at 5dpi were measured in Lrrk2 ${ }^{\mathrm{G} 2019 \mathrm{~S}}$ (B) and Lrrk2 KO cohorts (D). Each CFU data point represents one animal. Survival statistical analysis was done using the log-rank (Mantel-Cox) test. CFU analysis was statistically tested using one-way ANOVA with Tukey comparison tests. Error bars represent mean $\pm \mathrm{SEM}$ (data were generated by Bojan Shutinoski, Ph.D., and published in Shutinoski et al., 2019). 


\subsection{The Role of LRRK2 Kinase in Innate Immune Function}

The goal of this thesis was to evaluate the role of LRRK2 kinase activity in the innate immune system. The information from this study is critical for many reasons: (1) to inform the safety of LRRK2 kinase inhibitors as a treatment for PD patients; (2) to begin to elucidate the mechanisms through which LRRK2, and its associated kinase activity, function in the immune system, and (3) to gain insight into the etiology of PD to create cause-directed treatments for PD patients.

The underlying premise of my research was that a microbial infection is necessary to unmask a LRRK2-dependent phenotype. The specific hypothesis of this project was that LRRK2 kinase activity is required for its function in the innate immune system and that genetically or pharmacologically inhibiting the kinase function of LRRK2 will ablate the protective effect of LRRK2 in response to infection. Secondly, I postulated that LRRK2 functions in immune responses primarily in the periphery rather than in the brain. 


\section{Methods}

\section{Mouse Colonies}

All mouse colonies were kept on a C57BL/6J background. Colonies were maintained with heterozygous breeding pairs. Mice with an engineered mutation in the kinase domain, p.D1994S, were first reported in Herzig et. al. (2011) and are characterized by having no Lrrk2 kinase activity. These mice will be referred to as Lrrk $2^{\mathrm{D} 1994 \mathrm{~S}}$ or Lrrk2 kinase-dead mice herein. Of note, these homozygous Lrrk2 $2^{\text {D1994S }}$ mice are denoted Lrrk2 $2^{\text {DS/DS }}$ in some figures, whereas Lrrk2 ${ }^{\text {D1994S }}$ heterozygous mice are denoted Lrrk2 ${ }^{\text {WT/DS }}$. Mice carrying the PD-linked, p.G2019S mutation are also used and are characterized in Herzig et. al. (2011). They have a knock-in p.G2019S mutation in the murine Lrrk2 gene and have increased kinase activity of the protein. They will be referred to as Lrrk2 ${ }^{\mathrm{G} 2019 \mathrm{~S}}$ mice herein. Animal experiments were performed under the guidelines by Canadian Councils on Animal Care and approved by the Ethics Board of the Animal Care Committee at the University of Ottawa. Experiments involving viral or bacterial infections were done in a containment level 2 biohazard animal facility.

\section{Respiratory Enteric Orphan Serotype 3 Dearing Virus}

Respiratory Enteric Orphan Serotype 3 Dearing Virus (reovirus T3D) stocks were created by Dr. Earl Brown (uOttawa) prior to the start of this project. The titres of the viral stocks were confirmed by standard plaque assay upon beginning this project. Reovirus T3D is a ubiquitous, neurotropic virus that will reach the brain and cause encephalitis (Gauvin et al., 2013). In suckling pups, this encephalitis can be fatal, and therefore allows researchers to measure survival in a young pup model. When administered systemically, reovirus travels to the brain via neuronal spreading 
and hematogenous routes. Virus from the periphery will reach the brain beginning at 3 days postinfection (dpi) (Gauvin et al., 2013).

\section{Intranasal Reovirus T3D Infection}

Breeders were paired and expected birth dates were recorded. Mice were monitored the week prior to the expected birth, and once born, the pups were infected one day after birth (p1). Mouse pups were infected under isoflurane anesthesia using a p10 pipette to administer $5 \mu \mathrm{L}^{*}$ of reovirus T3D at a dose of $2.7 \times 10^{5}$ (which has been previously defined as one $\mathrm{LD}_{50}$, although was not seen in our Lrrk $2^{\text {D1994S }}$ experiment) directly to the nose pad. Mice were monitored until all the liquid was inhaled. Mice were returned to the parent cage following infection. These infected mice were used for two main readouts: survival and viral titre measurement (measured via plaque forming units assays (PFU)). For survival, mice were monitored twice daily to check for moribund state (the predetermined humane endpoint) (Fig. 2.1A). The moribund state was identified as inability to walk or to stay upright. Pairings were done either in heterozygous pairings, wild-type homozygous pairings, or Lrrk $2^{\mathrm{D} 1994 \mathrm{~S}}$ homozygous pairings where the researcher was blinded, to minimize bias.

For viral titre analysis, mice were culled at $3 \mathrm{dpi}$ or 11 dpi and brain and lungs were collected. $3 \mathrm{dpi}$ is peak viral titre in the lungs following nasal reovirus infection, and $11 \mathrm{dpi}$ is peak viral titre in the brain (Gauvin et al., 2013). Therefore, the time points 3dpi and 11dpi would be best representative for viral titres in the lung and brain, respectively. Mice were sacrificed via rapid decapitation and organs were collected and snap frozen on dry ice. Organs were then stored in the $-80^{\circ} \mathrm{C}$ freezer. 
*Note: for PFU analysis of $\operatorname{Lrrk} 2^{\mathrm{G} 2019 \mathrm{~S}}$ mice, mice were infected with $10 \mu \mathrm{L}$ of virus, at the same dose and procedure. Organ collection and homogenization was the same as for Lrrk2 ${ }^{\mathrm{D} 1994 \mathrm{~S}}$ mice.

\section{Plaque Assay}

Organs for plaque assay were homogenized in PBS at either a 3-fold (11dpi brains (intranasal); 8dpi brains (intracerebral)) or 5-fold dilution (11dpi lungs and 3dpi brains and lungs (intranasal); 8dpi liver and 3dpi brain and liver (intracerebral)). Samples were homogenized with metal beads using the MagnaLyser machine. Samples went through one freeze-thaw cycle in liquid nitrogen following homogenization. For the plaque assay, homogenates were used for serial dilutions and overlaid onto L929 cells in 6-well plates. Infected cells were overlaid with $2 \%$ purified again and 2x199 media in a 1:1 ratio. Plaques were identified using 0.015\% neutral red stain and manually counted. PFU was reported as plaques per gram (based on organ weight and original PBS dilution).

\section{Intracerebral Reovirus T3D Infection}

For intracerebral injections, purified reovirus, serotype-3 Dearing (abbreviated as T3D) was used. Mice were paired as per intranasal infections. Mice were inoculated one or three days ( 1 or $\mathrm{p} 3$, respectively) after birth. Mice were anesthetized under isoflurane and using a 30-gauge $50 \mu \mathrm{L}$ fixed needle Hamilton syringe, $10 \mu \mathrm{L}$ of virus at a dose of $5 \times 10^{2}$ PFU was injected into the left hemisphere of the pup cranium (frontal lobe) (Passini et al., 2001). Mice were then returned to the parent cage. Two main readouts were used for intracerebral injections: survival (measured as 
time-to-death) and measurement of viral titre as was described above. For all viral titre (PFU) analysis, mice were inoculated at p1 (Fig. 2.1B).

For viral titre analysis, mice were culled at either 3dpi or 8dpi and brain and liver were collected. In the intracerebral paradigm, mice begin to die rapidly at 9dpi, therefore $8 \mathrm{dpi}$ is the predetermined time point to collect organs. The time point 3dpi was added to investigate any differential effects on viral titre at early stages of disease. The liver was collected as a measure of migration of reovirus beginning in the brain to a peripheral organ (Gauvin et al., 2013). Mice were sacrificed via rapid decapitation and organs were collected and snap frozen on dry ice. Organs were then stored in the $-80^{\circ} \mathrm{C}$ freezer and later homogenized in the same fashion as intranasal inoculation samples.

\section{Salmonella typhimurium Infection}

Female mice (age 8-12 weeks) were infected with Salmonella typhimurium (ST) at a dose of 200 CFU. The mice were infected intravenously via the lateral tail vein with the help of Dr. B. Shutinoski. Mice were monitored for humane endpoint, described as when the mice could no longer walk properly and had a hunched up appearance. In a separate cohort, female mice were culled at $5 \mathrm{dpi}$ and spleens were collected for colony forming unit (CFU) analysis. This was done by standard CFU counting assay by Dr. B. Shutinoski (Fig. 2.1C). Female mice were used specifically for this experiment as the lab had previously identified a Lrrk2 female sex bias, whereby female mice carrying Lrrk2 ${ }^{\mathrm{G} 2019 \mathrm{~S}}$ mutations, and Lrrk2 knockout female mice tend to be more affected by infections than males. 


\section{Macrophage Culture}

Bone marrow-derived macrophages (BMDM) were used as a primary immune cell model to investigate Lrrk2-associated immune mechanisms, in vitro. Bone marrow was flushed from hind legs of adult mice and plated on $100 \mathrm{~cm}^{2}$ plate and differentiated to macrophages via M-CSF at 5ng/mL. Cells were grown in R8 media (RPMI 1640 with $8 \%$ fetal bovine serum and $50 \mu \mathrm{M} \beta$ mercaptoethanol). Media was changed every two days and macrophages were used for experiments on day 7. Cells were plated at $10^{6}$ cells $/ \mathrm{mL}$. BMDMs were then used for a variety of purposes, including cell viability assays, protein samples for Western blot, and plaque assay. Samples have also been collected for future experiments, including RNA analysis and cytokine and chemokine analysis.

\section{Cell Viability Assay}

BMDMs were treated with reovirus T3D at several multiplicity of infections (MOIs). Survival of macrophages following treatment was measured via cell viability neutral red assay. Cells were infected for 24 hours and neutral red was applied for two hours. The assay followed the Sigma protocol TOX4.

\section{Plaque Assay for BMDMs}

For viral titre analysis of BMDMs, cells were treated with reovirus for 24 hours. Cells were then lysed in media via three freeze-thaw cycles. This sample was then used for serial dilutions and used to treat L929 cells in the same manner as with the intranasal PFU paradigm. 


\section{Western Blotting}

For BMDM sample protein collection: cells were lysed in RIPA buffer in the presence of protease and phosphatase inhibitors and centrifuged at 14,800 RPM for 10 minutes. Protein concentration was determined using a BCA assay and samples were normalized for equal loading. Freezing of samples prior to sample preparation in loading dye was avoided as LRRK2 protein is prone to degradation with freeze-thaw. Fifteen- $\mu$ g of protein sample was run on the gel with NuPage loading dye.

For organ samples: following PBS homogenization, cells were treated with $1 \%$ Triton$\mathrm{X} 100,5 \mathrm{X}$ protease inhibitors, and 2X phosphatase inhibitors and spun at 14,800 RPM for 30 minutes at $4^{\circ} \mathrm{C}$. The supernatant was used as the protein sample. The protein concentration was calculated using BCA analysis, and normalized to $40 \mu \mathrm{g}$ to be run on the gel. These samples were mixed with NuPage loading dye and PBS buffer.

Westerns were run on $10 \%$ SDS gels in MOPS buffer. Gels were then transferred to a PVDF membrane using a standard semi-dry transfer in TRIS-glycine-Methanol buffer. The following antibodies were used: anti-actin (sc-81178, Santa Cruz; 1:1000 in 5\% BSA), antiSTAT1 (9172, Cell Signaling; 1:1000 in 5\%BSA), anti-p-STAT1-S727 (clone: D3B7, 8826, Cell Signaling; 1:1000 in 5\%BSA), anti-LRRK2 (MJFF2 c41-2, ab133474, AbCam; 1:1000 in 5\%BSA), anti-p-LRRK2-S935 (UDD2 10(12), ab133450, AbCam; 1:1000 in 5\% BSA). Western blots were imaged using BioRad ChemiDoc Imager. 


\section{Statistical Analysis}

All statistical analysis was performed in GraphPad Prism 5. For survival assays, the logrank (Mantel-Cox) test was used. The Mann-Whitney test was used to test statistical differences in PFU assays, and unpaired t-test was used in the analysis of in vitro data. Data are demonstrated as mean \pm SEM where applicable and as described in the figure legends.

(A)

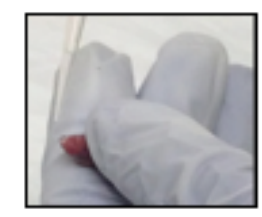

(B)

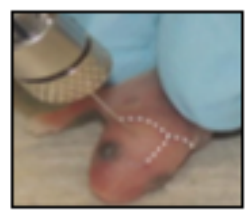

(C)

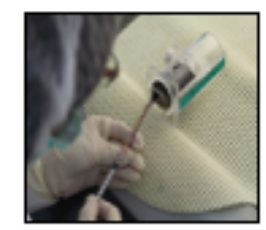

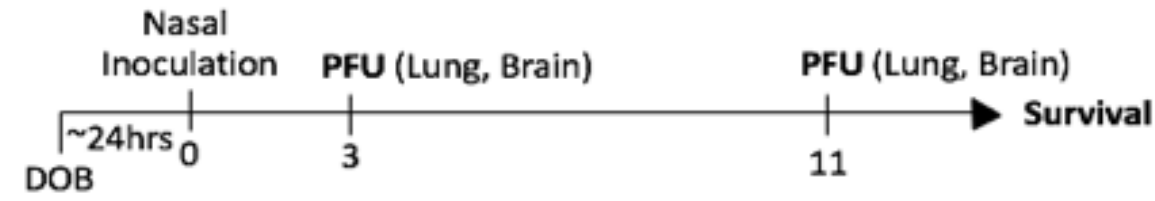
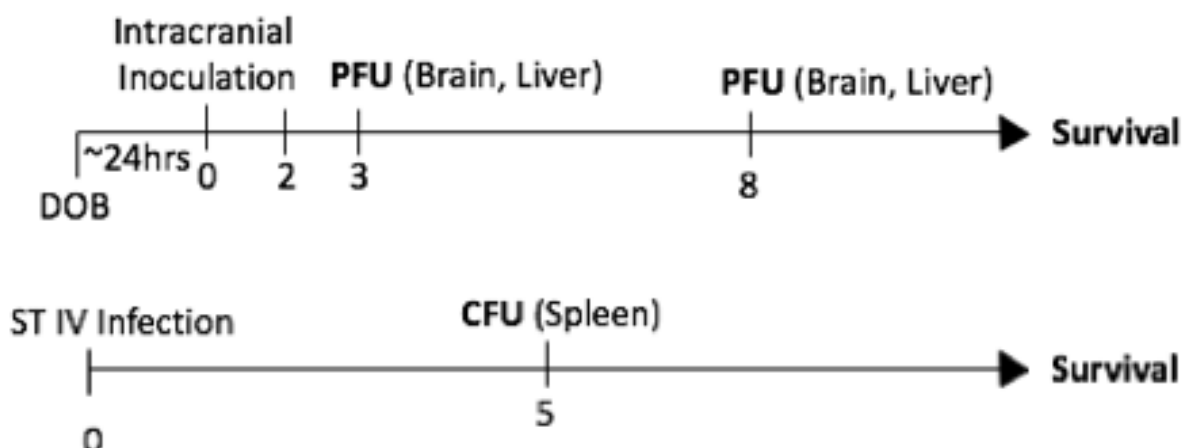

0

\section{Survival}

Fig. 2.1: Schematic representation of the experimental paradigms employed in this thesis. A nasal inoculation paradigm of $\mathrm{p} 1$ pups with reovirus T3D to model a systemic, viral infection (A); an intracerebral inoculation paradigm of $\mathrm{p} 1$ or $\mathrm{p} 3$ pups with reovirus T3D to model a direct-brain infection (B); and an intravenous bacterial infection paradigm used in adult mice to model a peripheral, bacterial infection (C). 


\section{Results}

Lrrk2 is expressed in wild-type mice as early as p1 in the brain and spleen, and Lrrk2 expression appears to increase with age in the brain.

Mouse pups as young as postnatal day $1(\mathrm{p} 1)$ were used for viral inoculation paradigms. We therefore validated the presence of wild-type Lrrk2 in the brain and spleen of wild-type pups (both male and female) by Western blot (n=3) (Fig. 3.1 A, B). We have confirmed that wild-type Lrrk2 was found in young animals at p1 in both the brain and spleen (Fig. $3.1 \mathrm{~A}, \mathrm{~B}$ ). In brain samples, we measured the expression of Lrrk2 with age in p1 to p21 uninfected wild-type samples (Fig. 3.1 A, B). We detected that Lrrk2 levels in the brain increased over the first 21 days, where it reached the level that is observed in wild-type, adult mice, suggesting that it reached a plateau (Fig. 3.1B). Additionally, it did not appear that the levels of Lrrk2 differ between males and females at these time points. 
(A)

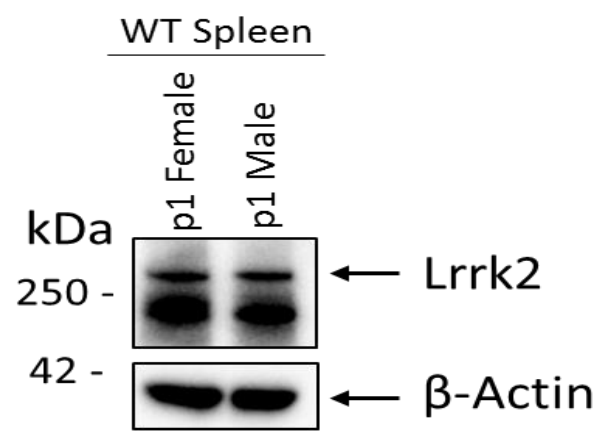

(B)

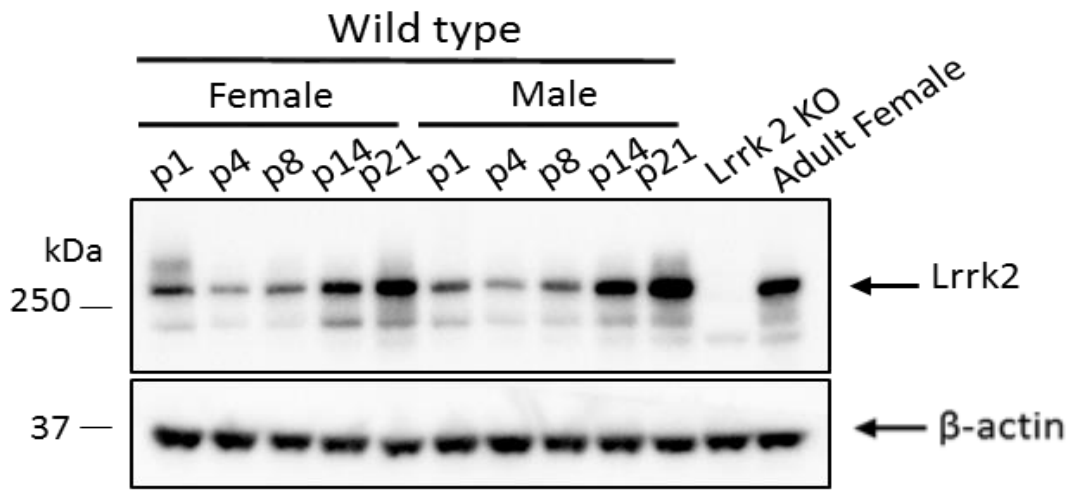

Fig. 3.1: Lrrk2 is present in wild-type spleens as early as p1, and its detection increases in the brain with age. Western blot for Lrrk2 $(286 \mathrm{kDa})$ from female and male wild-type spleen homogenates at p1 (n=3) (A), and male and female brain homogenates from p1 to $\mathrm{p} 21(\mathrm{n}=3)(\mathrm{B})$. $\beta$-actin is visualized as a loading control. 


\section{Lrrk2 $2^{62019 S}$ mice clear pathogens from the brain and lungs more effectively than wild-type animals.}

To test the effects of the Lrrk2 in the innate immune response, we employed an acute, viral infection paradigm in suckling pups in order to illicit the innate immune response (i.e., p1 pups do not have an established adaptive immune response yet). In this paradigm, mice were infected 24 hours after birth with reovirus T3D administered to the nose pad. The virus was then inhaled by the pup and will move from the periphery to the brain beginning by $3 \mathrm{dpi}$, where it will cause encephalitis (Fig. 2.1A).

Previous studies in the lab had shown that Lrrk2 was required for a host to properly respond to a virulent pathogen because Lrrk2 $\mathrm{KO}$ mice demonstrated higher mortality in the reovirus T3D paradigm (Fig. 1.2A); furthermore, its kinase activity conferred a protective effect, as the Lrrk2 ${ }^{\mathrm{G} 2019 \mathrm{~S}}$ mice (with increased enzymatic activity) had improved survival in a bacterial sepsis paradigm (Fig. 1.4) (Shutinoski et al, 2019). To determine if the same was true for mice carrying the p.G2019S mutation in the chosen viral inoculation paradigm, infections were repeated in new cohorts of wild-type and Lrrk2 ${ }^{\mathrm{G} 2019 \mathrm{~S}}$ mice, and organs were collected at 3dpi and $11 \mathrm{dpi}$ for viral load analysis (PFU). Note, in this paradigm, siblings were used from either wild-type or Lrrk2 ${ }^{\mathrm{G} 2019 \mathrm{~S}}$ homozygous pairings respectively.

As observed in the Salmonella sepsis paradigm, we found that Lrrk2 ${ }^{\mathrm{G} 2019 \mathrm{~S}}$ mice had lower viral titres in the lungs at peak day 3 post infection, and in the brains at peak day 11 post infection (Fig. 3.2). The mean viral titre in lungs of wild-type mice at 3 dpi was $1.4 \times 10^{7} \mathrm{PFU} / \mathrm{g}(\mathrm{n}=7)$, whereas the mean viral titre at $3 \mathrm{dpi}$ for $\operatorname{Lrrk} 2^{\mathrm{G} 2019 \mathrm{~S}}$ mice was $2.48 \mathrm{X} 10^{6} \mathrm{PFU} / \mathrm{g}(\mathrm{n}=3)$. In comparison, at $11 \mathrm{dpi}$, the mean viral titre in the brain of wild-type mice was $8.64 \times 10^{8} \mathrm{PFU} / \mathrm{g}$ 
$(\mathrm{n}=10)$, and the mean viral titre of Lrrk2 ${ }^{\mathrm{G} 2019 \mathrm{~S}}$ mice was $3.45 \times 10^{8} \mathrm{PFU} / \mathrm{g}(\mathrm{n}=18)$. Statistical significance was measured by Mann-Whitney test (3dpi p-value: 0.0218; $11 \mathrm{dpi}$ p-value: 0.0262 ). We concluded that the p.G2019S mutant conferred a more effective control over the replication rate of both Salmonella (Shutinoski et al., 2019) and virulent reovirus-T3D during the height of their infectivity. 

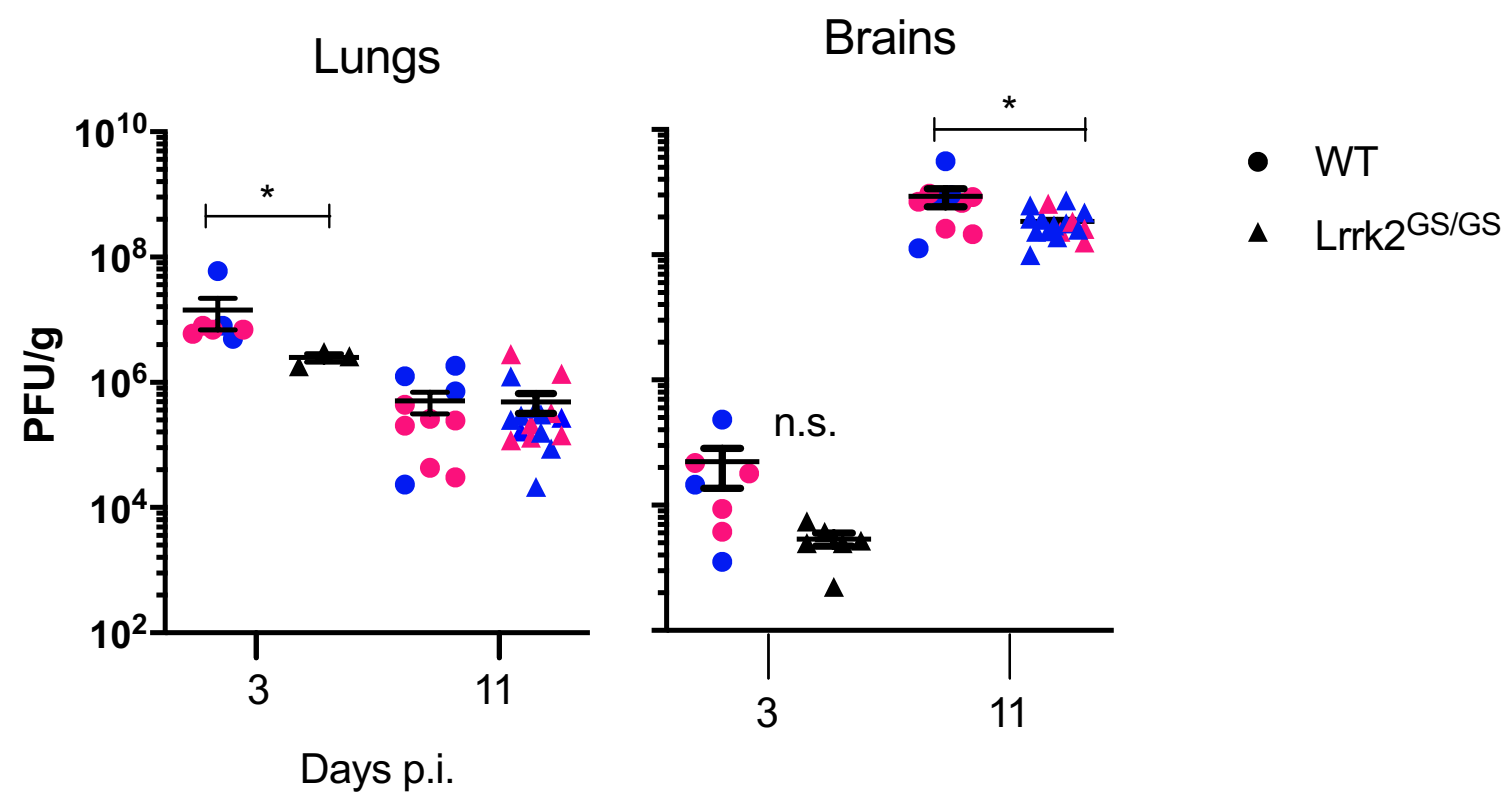

Fig. 3.2: Lrrk2 ${ }^{\mathrm{G} 2019 \mathrm{~S}}$ mice have lower viral titres in the brain and lungs following reovirusT3D nasal infection compared to wild-type mice. Brains and lungs were collected from nasally infected mice (dose: $2.7 \times 10^{5} \mathrm{PFU}$ ) and collected at 3 and $11 \mathrm{dpi}$. Viral titre was measured as plaque forming units (PFU) in lysates of whole organs. PFU counts of wild-type and Lrrk2 $2^{\mathrm{G} 2019 \mathrm{~S}}$ lungs and brains are graphically displayed at 3and 11dpi, where each symbol represents one animal. Females are represented in pink and males are represented in blue. Of note, samples denoted in black were lost after genotyping for Lrrk2, and therefore sex could not be identified. Wild-type 3dpi lungs $(n=7)$ and 11dpi lungs $(n=10)$; Lrrk2 ${ }^{\text {G2019S }} 3$ dpi lungs $(n=3)$ and 11 dpi lungs ( $\mathrm{n}=18)$; Wild-type 3 dpi brain $(\mathrm{n}=7)$ and $11 \mathrm{dpi}$ brain $(\mathrm{n}=10)$; Lrrk2 $2^{\mathrm{G} 2019 \mathrm{~S}} 3 \mathrm{dpi}$ brain $(\mathrm{n}=6)$ and $11 \mathrm{dpi}$ brain $(\mathrm{n}=17)$. Lungs $3 \mathrm{dpi} \mathrm{p}$-value $=0.0167$; brain $11 \mathrm{dpi} \mathrm{p}$-value $=0.0262$. ${ }^{*} \mathrm{P} \leq 0.05$. Test used: non-parametric Mann-Whitney test. Error bars represent mean \pm SEM. 
Lrrk $2^{\text {D1994S }}$ mice (kinase-dead) show lesser viral clearance at the early stages of disease compared to wild-type controls, but show increased survival in a viral brain infection paradigm.

The results from the Lrrk2 ${ }^{\mathrm{G} 2019 \mathrm{~S}}$ mutant mice suggested that Lrrk2 kinase activity promoted an enhanced immune response. We therefore sought to elucidate if its kinase activity was required for its role in the innate immune response by employing a Lrrk2 kinase-dead mouse model. These mice have an engineered knock-in p.D1994S mutation in the kinase domain of Lrrk2 (Herzig et al., 2011). In initial reports, it is mentioned that Lrrk2 with the p.D1994S mutation is less stable than wild-type Lrrk2 (Herzig et al., 2011). Therefore, we first performed Western blot analysis to demonstrate the levels of Lrrk2 in the organs of mice employed in this paradigm. We also measured the levels of phospho- Ser935 Lrrk2 (indicative of autophosphorylation by Lrrk2 itself (Steger et al., 2016)) as a measure of Lrrk2 kinase activity. We used samples that had been collected and homogenized from intranasally inoculated reovirus T3D-infected mice, as described above. Brains and lungs were collected at $3 \mathrm{dpi}$ and at $11 \mathrm{dpi}$ which represent timepoints of expected peak viral titres for the respective organs (Gauvin et al., 2013). We found that Lrrk2 was equally expressed between wild-type mice and kinase-dead in these organs at these time points following infection in both females and males (n=3) (Fig.3.3 A, B).

Additionally, we confirmed that Ser935-phosphorylated Lrrk2 was significantly reduced in kinase-dead mutant tissue, suggesting that the Lrrk2 kinase activity was indeed markedly diminished in the Lrrk2 ${ }^{\text {D1994S }}$ mice (Fig. 3.3 A, B), as was shown in the original report (Herzig et al., 2013). Lrrk2 residue Ser-935 is also phosphorylated by kinases of the IкB family, which could explain the weak, residual phospho-Ser935 Lrrk2 signal in the Lrrk2 kinase-dead homogenates (Dzamko et al., 2012). (Note, the issue of residual kinase activity in this context is being further 
confirmed in collaboration with Dr. Dario Alessi (University of Dundee) using in vitro Lrrk2 kinase assays). We also confirmed that the protein was present and abundant in uninfected Lrrk2 ${ }^{\mathrm{D} 1994 \mathrm{~S}}$ mice at $\mathrm{p} 1, \mathrm{p} 4$, and $\mathrm{p} 21$. This confirmed that Lrrk2 protein expression was not infection-dependent at the ages relevant in this paradigm, because Lrrk2 was present at each of these timepoints ( $\mathrm{n}=1)$ (Fig. 3.3C). 
(A)

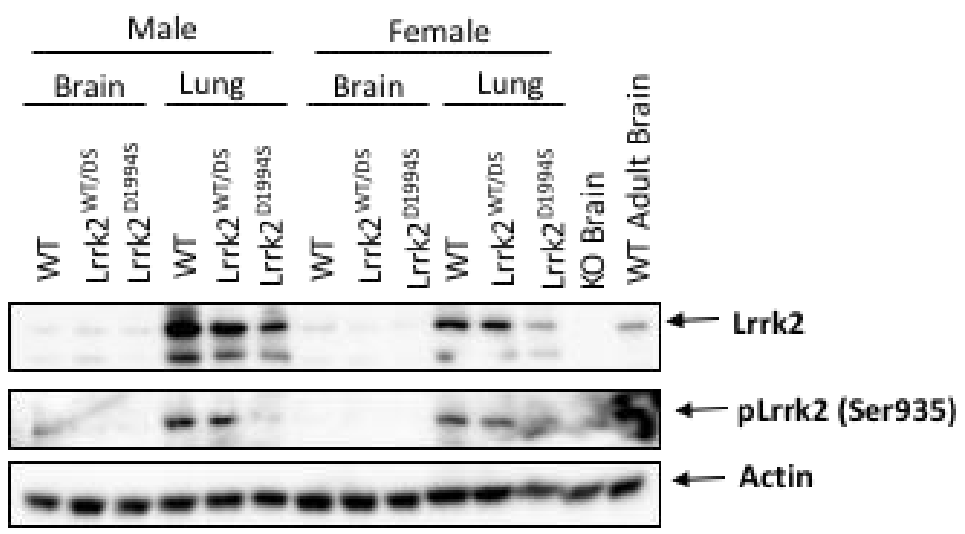

$3 \mathrm{dpi}$

(B)

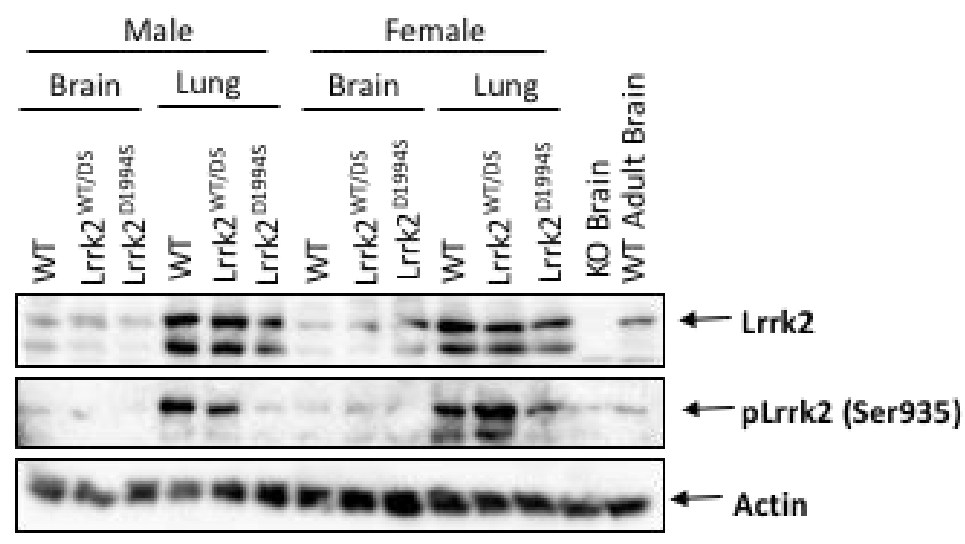

$11 \mathrm{dpi}$

(C)

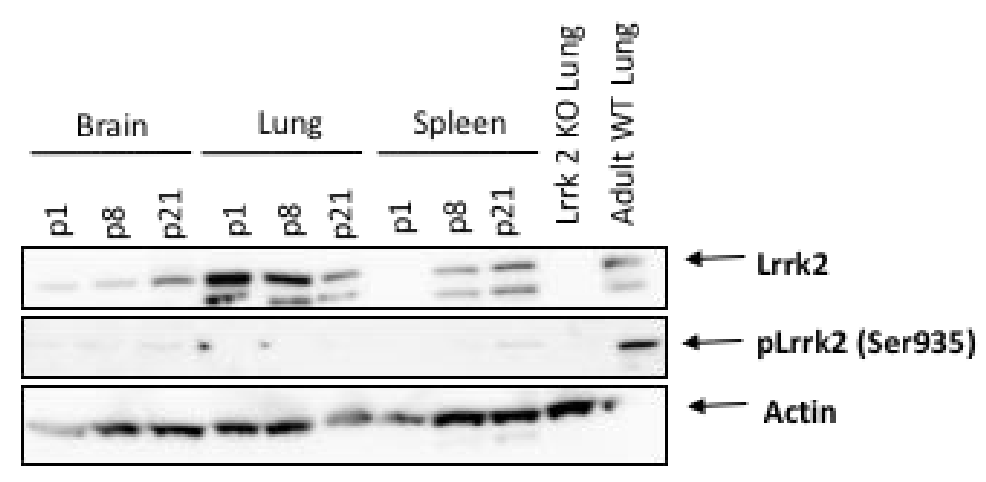

Fig. 3.3: Lrrk2 ${ }^{\text {D1994S }}$ mutant mice have equal Lrrk2 protein expression but no kinase activity in brain and lung compared to wild-type mice following reovirus infection. Newborn mice were nasally inoculated with one $\mathrm{LD}_{50}$ of reovirus-T3D, as described in the Method section, leading to infection. At 3dpi and 11dpi, brains and lungs were collected from wild-type, 
Lrrk2 ${ }^{\text {D1994s }}$, and heterozygous animals and homogenized for Lrrk2 Western blot analysis in females (n=3) (A) or males (n=3) (B). Brain and lung were also collected from uninfected $\operatorname{Lrrk2}^{\mathrm{D} 1994 \mathrm{~S}}$ mice $(\mathrm{p} 1, \mathrm{p} 4, \mathrm{p} 21)$ to measure Lrrk2 expression via Western blot $(\mathrm{n}=1)(\mathrm{C}) . \beta$-actin is immunoblotted as a loading control. 
Having established that the protein was present in Lrrk2 ${ }^{\mathrm{D} 1994 \mathrm{~S}}$ organs and at comparable levels to wild-type, we next employed the viral nasal paradigm in mice expressing the Lrrk2 ${ }^{\text {D1994S }}$ mutation, exactly as has been done with Lrrk2 KO mice and Lrrk2 ${ }^{\mathrm{G} 2019 \mathrm{~S}}$ mice. We administered one $\mathrm{LD}_{50}\left(2.7 \times 10^{5} \mathrm{PFU}\right)$ of reovirus $\mathrm{T} 3 \mathrm{D}$ to each mouse. Mice were bred in heterozygous pairings and therefore we tested littermates.

I had hypothesized that the Lrrk2 kinase activity was critical to its role in the immune system, and that by genetically knocking out this activity, these mice would be significantly more susceptible to systemic infection with reovirus, as was seen in the Lrrk2 knockout mice. Remarkably, the Lrrk2 kinase-dead mice had significantly higher survival than wild-type littermates, with the heterozygous mice falling between the two homozygous genotypes (Fig. 3.4). Sample sets were: WT $(n=13)$; $\operatorname{Lrrk} 2^{\text {WT/DS }}(n=28) ; \operatorname{Lrrk}^{\text {D1994S }}(n=15)$. The earliest an animal succumbed to infection was 9 dpi $\left(\operatorname{Lrrk} 2^{\mathrm{WT} / \mathrm{DS}}\right)$, with the last time point at which an animal succumbed to infection being $25 \mathrm{dpi}\left(\operatorname{Lrrk} 2^{\mathrm{D} 1994 \mathrm{~S}}\right)$. Mice were considered survivors after 30dpi. Of the mice that had died, majority did so on 9, 10, and 11dpi. As for survivors, wild-type mice had a $\sim 10 \%$ survival rate, heterozygous mice had $\sim 20 \%$ survival rate, and Lrrk $2^{\mathrm{D} 1994 \mathrm{~S}}$ mice had $\sim 50 \%$ survival rate. This result suggested that not only is the Lrrk2 kinase activity not essential in the innate immune response, but it may be protective, at least in the context of this systemic dsRNA virus infection paradigm. By comparing these results to the previous results in which Lrrk2 knockout mice had decreased survival, this also indicates that the Lrrk2 protein, but not its kinase activity, is important in functions related to innate immune responses by the host. 


\section{Intranasal Inoculation}

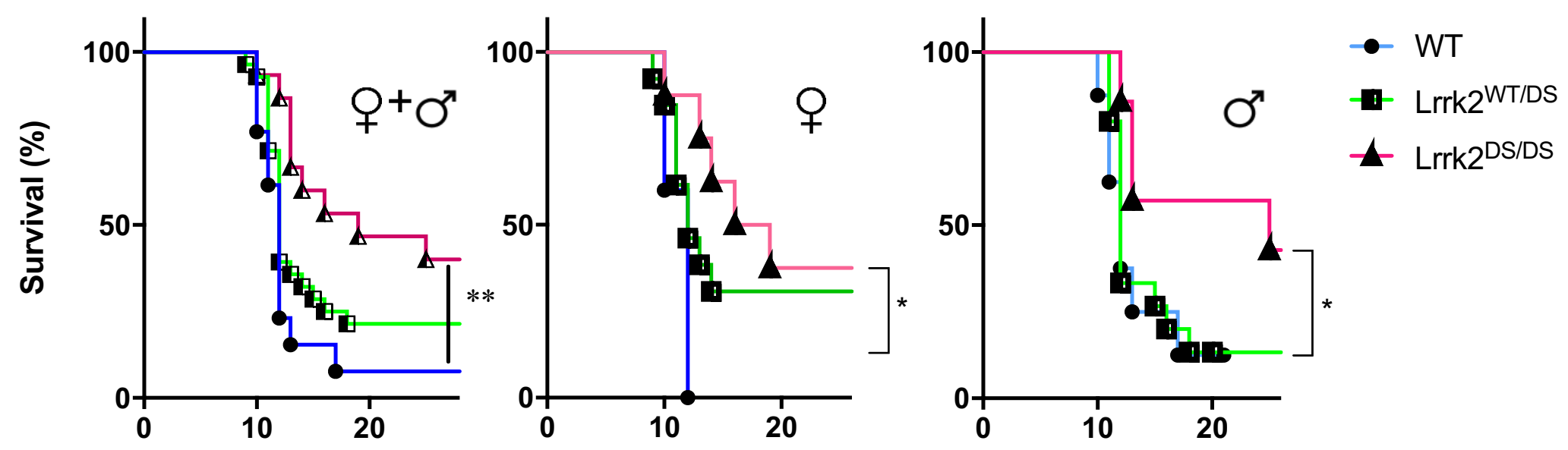

\section{Days Post Infection}

Fig. 3.4: Lrrk2 ${ }^{\text {D1994S }}$ mutant mice show improved survival from encephalitis with a female sex bias compared to wild-type mice. Newborn mice were nasally inoculated with one $\mathrm{LD}_{50}$ of reovirus-T3D, as described in the Method section, leading to infection. Lrrk2 ${ }^{\text {D1994S }}$ and wild-type mice were measured for survival following infection, and separated based on sex. WT n=13 (f:5; $\mathrm{m}: 8) ; \operatorname{Lrrk} 2^{W T / D S} n=28$ (f:15; m:13); $\operatorname{Lrrk}^{\mathrm{DS} / \mathrm{DS}} \mathrm{n}=15$ (f:8; m:7). Data were analyzed using the logrank (Mantel-Cox) test. The p-value between WT and Lrrk2 $2^{\mathrm{D} 1994 \mathrm{~S}}=0.0030$ when sexes are pooled; in females alone $\mathrm{p}$-value $=0.046$; in males alone $\mathrm{p}$-value $=0.0430 .{ }^{*} \mathrm{P} \leq 0.05,{ }^{*} * \mathrm{P} \leq 0.01$. Note, graphically wild-type mice are denoted WT; Lrrk2 ${ }^{\text {D1994S }}$ heterozygous mice are denoted Lrrk2 ${ }^{\text {WT/DS}}$; Lrrk2 $2^{\text {D1994S }}$ homozygous mice are denoted Lrrk2 ${ }^{\text {DS/DS }}$. 
To determine whether this change in survival was associated with a better control of viral replication by the Lrrk2 ${ }^{\mathrm{D} 1994 \mathrm{~S}}$ mice, we measured viral titres (PFU/g) in the brain and spleens of wild-type, heterozygous, and Lrrk2 ${ }^{\text {D1994S }}$ littermates. There, we found that Lrrk2 ${ }^{\text {D1994S }}$ mice had significantly greater viral titre in the lungs at 3 dpi compared to wild-type controls, but showed no difference in viral titres in the brain at $11 \mathrm{dpi}$. At $3 \mathrm{dpi}$, the mean viral titre in wild-type lungs was $1.99 \times 10^{6} \mathrm{PFU} / \mathrm{g}(\mathrm{n}=8)$, in heterozygous lungs was $1.98 \times 10^{6} \mathrm{PFU} / \mathrm{g}(\mathrm{n}=10)$, and Lrrk2 ${ }^{\mathrm{D} 1994 \mathrm{~S}}$ lungs was $1.80 \times 10^{7}(\mathrm{n}=13)$. The Mann-Whitney test was used to determine significance (WT vs. Lrrk2 ${ }^{\text {D1994S }}$ p-value: 0.0342 ; heterozygous $v s$. Lrrk2 ${ }^{\text {D1994S }}$ p-value: 0.0025$)$. The average PFU/g across genotypes in the brain at $3 \mathrm{dpi}$ was $7.08 \times 10^{3} \mathrm{PFU} / \mathrm{g}$; in the lung at $11 \mathrm{dpi}$ was $7.49 \times 10^{4}$ $\mathrm{PFU} / \mathrm{g}$; and in the brain at $11 \mathrm{dpi}$ was $3.38 \times 10^{8} \mathrm{PFU} / \mathrm{g}$. These results suggested to us that Lrrk2 kinase activity may be important in viral clearance at early of stages of disease in the lungs, where the expression of Lrrk2 protein (and LRRK2 mRNA in humans) is at the highest level of detection; alternatively, reduced kinase activity could be remedied and compensated for at later stages when the disease process has entered the brain (and where the expression of Lrrk2 protein and LRRK2 mRNA in humans is at a low level of detection) (Fig. 3.5). Notably, this significant difference seems to be determined by females, as when sexes were separated, the significant difference between female Lrrk2 ${ }^{\text {D1994S }}$ mice and female wild-type mice was much stronger than between male Lrrk2 ${ }^{\text {D1994S }}$ mice and male wild-type mice, consistent with a previously established female sex bias with Lrrk2 variants (determined by Mann-Whitney test, p-value= 0.0283)(Fig. 3.6 A,B).

One possible mechanism is that Lrrk2 modulates the degree of ensuing inflammation as has been suggested in our previous Lrrk2-related studies. Future studies will investigate the degree of inflammation in Lrrk2 ${ }^{\mathrm{D} 1994 \mathrm{~S}}$ brains. This will be done as was previously done for the Lrrk2 ${ }^{\mathrm{G} 2019 \mathrm{~S}}$ and Lrrk2 knockout studies. Specifically, to employ flow cytometry to measure the relative 
abundance of infiltrating myeloid cells into infected Lrrk2 $2^{\mathrm{D} 1994 \mathrm{~S}}$ brains $v s$. wild-type brains. This will provide insight whether the protective function of kinase-dead Lrrk2 is due to an altered degree of infiltration by peripheral immune cells into the brain and therefore better control of subsequent inflammation. Additionally, immunohistochemistry will be employed to measure microglial activation in the brains of Lrrk2 kinase-dead and Lrrk2 wild-type mice following infection, and to anatomically measure and compare the abundance and spread of reovirus in the brains of each genotype. 


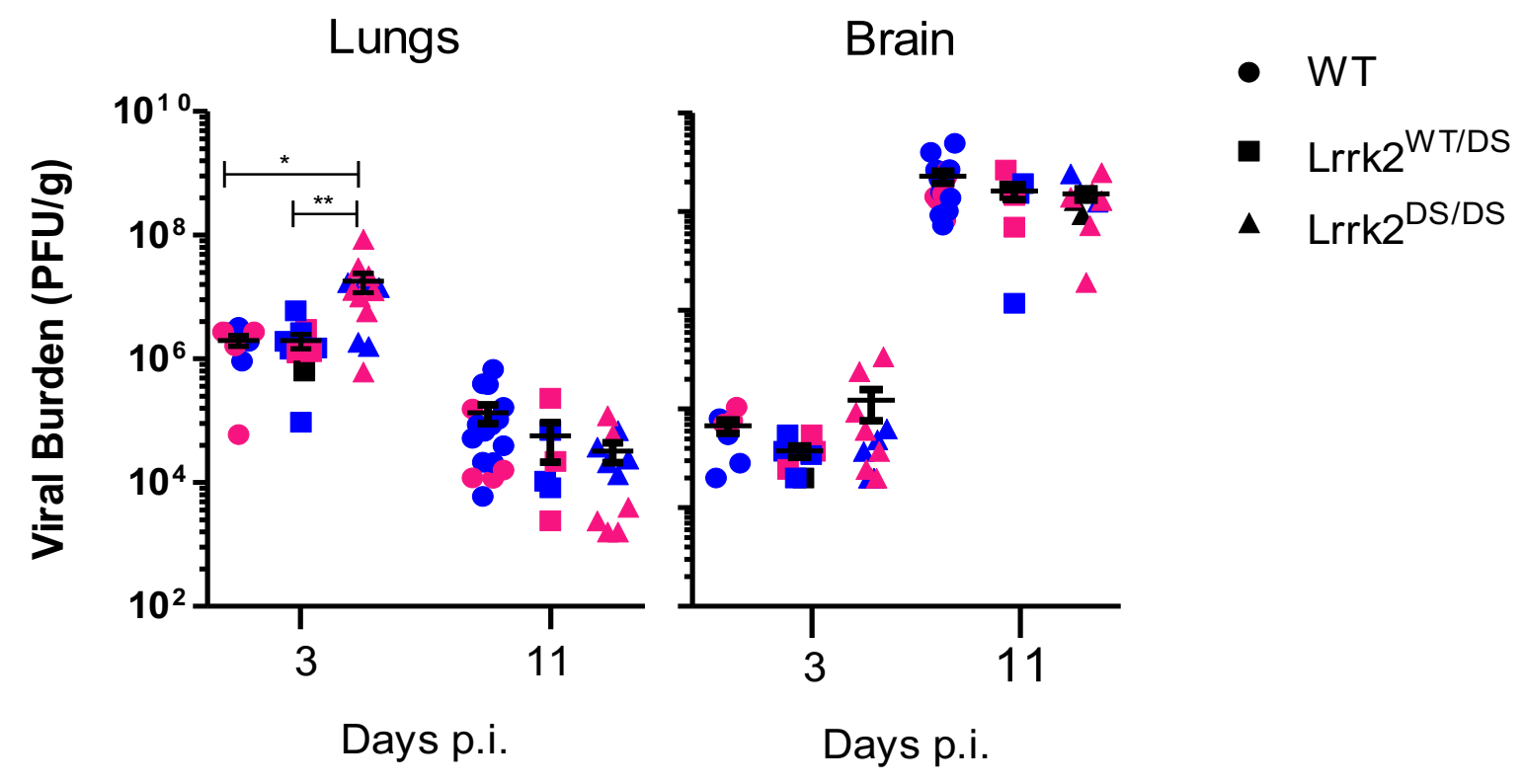

Fig. 3.5: Lrrk2 ${ }^{\text {D1994S }}$ mutant mice have increased viral burden in the lungs early in disease but show no change in viral burden at the late stage disease following a viral, nasal infection compared to wild-type mice. Newborn mice were nasally inoculated with one $\mathrm{LD}_{50}$ of reovirusT3D, as described in the Method section, leading to infection. Homozygous and heterozygous Lrrk $2^{\text {D1994S }}$ mice and wild-type mice were sacrificed at 3dpi and 11dpi and lungs and brain were collected and homogenized. Data are represented in PFU/g, where each symbol represents one animal. Pink data points indicate females, and blue data points indicate males. Wild-type 3dpi lungs $(n=8)$ and 11dpi lungs $(n=17)$; heterozygous 3dpi lungs $(n=10)$ and 11dpi lungs $(n=6)$; Lrrk $2^{\text {D1994S }}$ homozygous 3dpi lungs $(n=13)$ and 11dpi lungs $(n=11)$. Wild-type 3dpi brain $(n=7)$ and 11dpi brain $(n=18)$; heterozygous 3dpi brain $(n=8)$ and 11dpi brain $(n=7)$; Lrrk2 ${ }^{\text {D1994S }} 3$ dpi brain $(n=13)$ and 11dpi brain $(n=11)$. The $p$-value between WT and Lrrk2 ${ }^{\text {1994S }}$ in lungs at 3dpi $=0.0153$; between Lrrk2 ${ }^{\text {WT/DS }}$ Lrrk2 ${ }^{\text {D1994S }}$ p-value $=0.0039$. Data were statistically analyzed using the Mann-Whitney test. Error bars represent mean $\pm \mathrm{SEM} .{ }^{*} \mathrm{P} \leq 0.05, * * \mathrm{P} \leq 0.01$. Note, graphically wild-type mice are denoted WT; Lrrk2 ${ }^{\text {D1994S }}$ heterozygous mice are denoted Lrrk2 ${ }^{\text {WT/DS}}$; Lrrk2 $2^{\text {D1994S }}$ homozygous mice are denoted Lrrk2 ${ }^{\text {DS/DS }}$. 
(A)

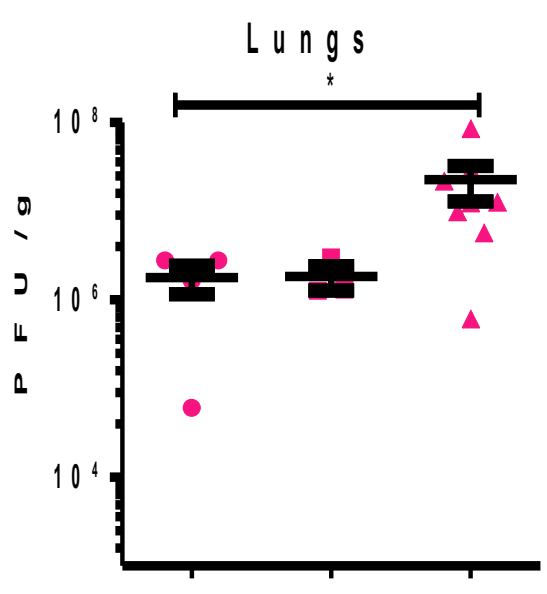

3 Days p.i.
(B)

- Lrk 2 W T/WT

- Lrkk TIDS

$\triangle \operatorname{Lrk} 2$ DSIDS

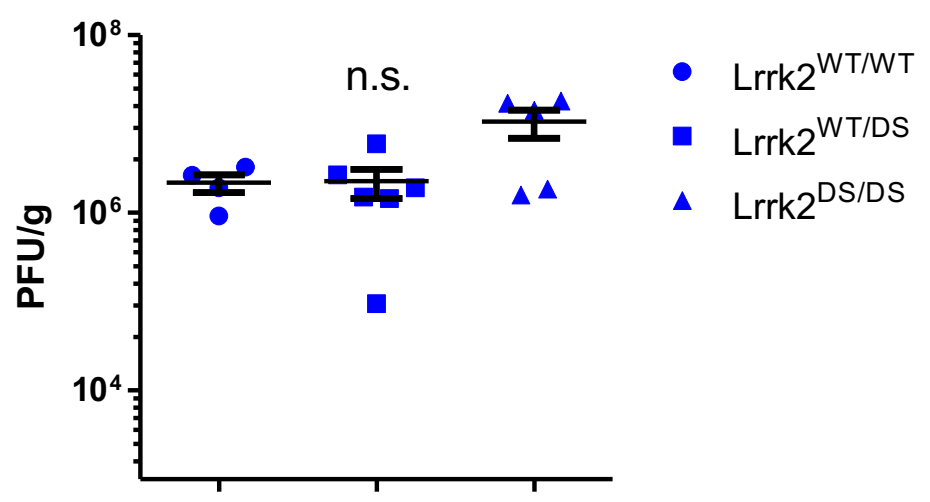

3 Days p.i.

Fig. 3.6: The increased viral burden in the lungs early in disease following a viral, nasal infection in Lrrk2 ${ }^{\text {D1994S }}$ mutant mice follows a female sex bias. Data from Fig. 3.5 (lungs at 3dpi) were separated into females (A) and males (B) to indicated further differences. The p-value between female wild-type lungs and Lrrk2 ${ }^{\mathrm{D} 1994 \mathrm{~S}}$ lungs $\mathrm{p}$-value $=0.0283$. Data were statistically analyzed using the Mann-Whitney test. Error bars represent mean $\pm \mathrm{SEM} . * \mathrm{P} \leq 0.05, * * \mathrm{P} \leq 0.01$. Note, graphically wild-type mice are denoted WT; Lrrk2 ${ }^{\text {D1994S }}$ heterozygous mice are denoted Lrrk2 ${ }^{\text {WT/DS}}$; Lrrk2 ${ }^{\text {D1994S }}$ homozygous mice are denoted Lrrk2 ${ }^{\mathrm{DS} / \mathrm{DS}}$. 
mutants.

To investigate the sex effect of Lrrk2 kinase activity in response to a viral infection, we separated the intranasal reovirus T3D survival curve based on sex for each genotype (Fig. 3.7). We found no significant differences between male and females animals that were homozygous for their Lrrk2 genotypes; however, there was an interesting trend with the heterozygous mice (Fig. 3.7B). The female heterozygous mice behaved similarly to the homozygous mutant mice (i.e., better survival) whereas the male heterozygous mice behaved more similar to the wild-types (i.e., worsened survival). This is similar to what was observed in our previous studies where the heterozygous Lrrk $2^{\mathrm{WT} / \mathrm{KO}}$ females behaved like the mutant $\mathrm{Lrrk} 2^{\mathrm{KO} / \mathrm{KO}}$ mice (i.e., worsened survival) and the heterozygous Lrrk $2^{\mathrm{WT} / \mathrm{KO}}$ males behaved like the wild-type mice (i.e., better survival) (Shutinoski et al., 2019). 
(A)

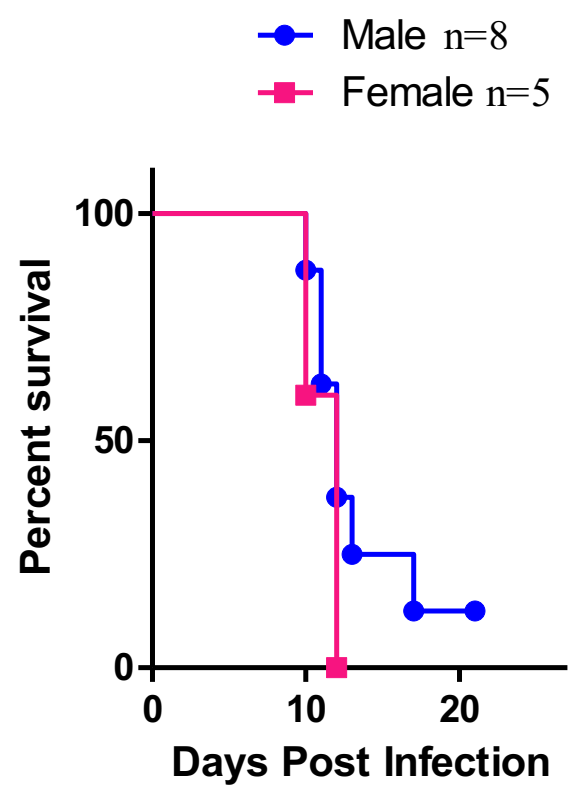

(B)

(C)
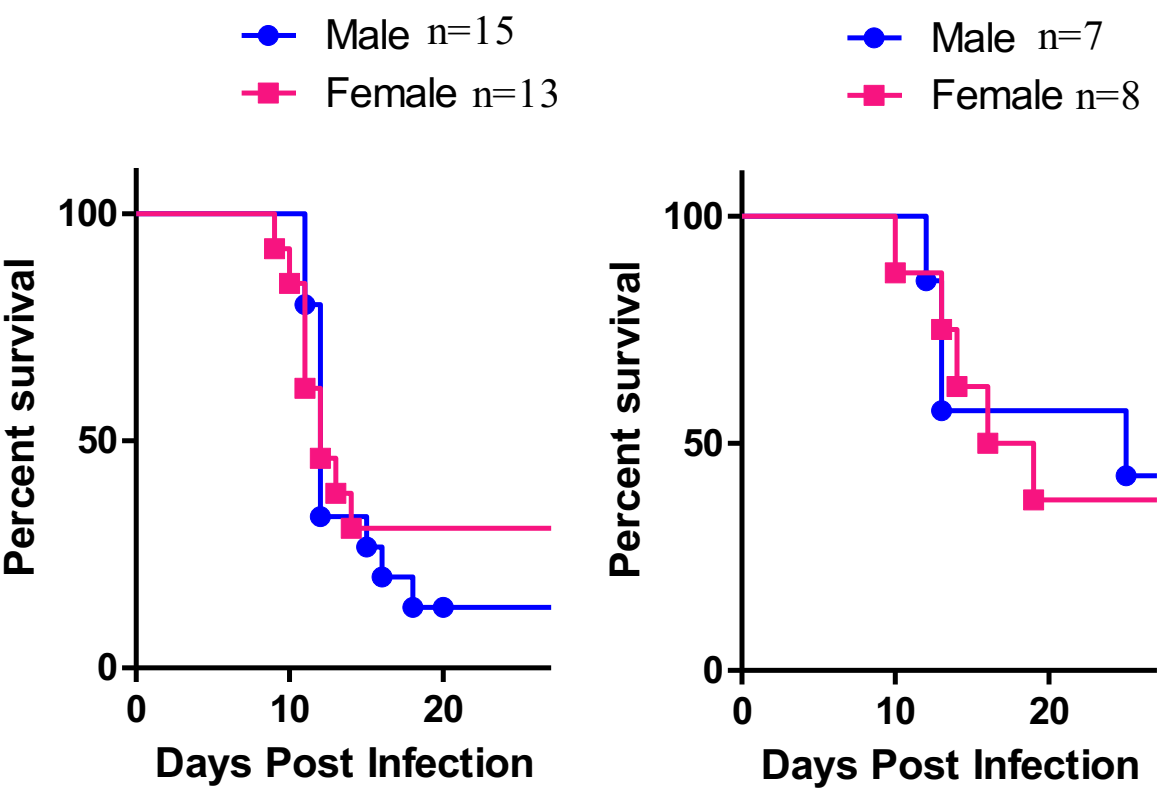

Fig. 3.7: Lrrk2 ${ }^{\mathrm{WT} / \mathrm{DS}}$ heterozygous mice show a sex bias as previously seen with other Lrrk2 variants. Newborn mice were nasally inoculated with one $\mathrm{LD}_{50}$ of reovirus-T3D and monitored for survival. These data were separated for sex based on each genotype: wild-type mice (A), Lrrk2 ${ }^{\text {D1994S }}$ heterozygous mice (B), or Lrrk2 ${ }^{\text {D1994S }}$ kinase-dead mice (C). Data were analyzed using the log-rank (Mantel-Cox) test. Sample set n-values are indicated on each graph. No significant differences were found in these survival curves. 


\section{Intracerebral inoculation: Lrrk2 kinase-dead mice have an equal survival rate as heterozygous and wild-type mice following direct infection of the brain with reovirus T3D, but have a decreased viral titre in the brain at the later stage of disease.}

The nasal route of infection leads to a systemic infection, i.e., viral infection of both peripheral organs and secondarily, of the brain, where the viral titres peak in the periphery first, and only become detectable in the brain at 3dpi (peaking at 11dpi) (Gauvin et al., 2013). Therefore, we do not know exactly how much of the virus reaches the brain in the early dissemination phase, and how much of the virus was sequestered in the periphery before reaching the brain, i.e., does Lrrk2 function to control the virus prior to reaching the brain, or once the virus has propagated in the brain?

To elucidate this, we employed an intracerebral injection paradigm (Fig. 2.1B). In this paradigm, $5 \times 10^{2}$ PFU of reovirus $\mathrm{T} 3 \mathrm{D}$ were administered via injection directly into the left hemisphere (frontal lobe) of the pup brain at p1 (Passini et al., 2001). Although we expect secondary, peripheral involvement (in the form of myeloid cell infiltration), the primary response will be brain-specific, thus allowing us to assess Lrrk $2^{\text {D1994S }}$ function in the brain independent of its potential role in the periphery. Two specific outcome measures were assessed: survival, reported as time-to-death as this is a lethal paradigm; and viral titres in infected organs. Note, in this paradigm, samples include littermates from heterozygous crossings, as well as samples from homozygous wild-type $\mathrm{x}$ wild-type pairings, and Lrrk2 $2^{\mathrm{D} 1994 \mathrm{~S}} \mathrm{x}$ Lrrk2 ${ }^{\mathrm{D} 1994 \mathrm{~S}}$ pairings.

Although we saw a stark survival benefit in the Lrrk2 $2^{\text {D1994S }}$ mice in the systemic, nasal inoculation-induced, viral paradigm, we saw no survival change following direct brain inoculation at p1 compared to wild-type controls (Fig. 3.8A). In fact, wild-type mice, heterozygous, and kinase-dead mice all had the same survival rate (measured by time-to-death). Specifically, mice 
began to die at $9 \mathrm{dpi}$, and were all dead by $13 \mathrm{dpi}$ (except one Lrrk2 ${ }^{\mathrm{D} 1994 \mathrm{~S}}$ mouse which died at 16dpi). Indeed, $90 \%$ of mice were dead by 11 dpi. The sample sets were: WT $(n=20)$; Lrrk2 ${ }^{\text {WT/DS }}$ $(\mathrm{n}=11) ; \operatorname{Lrrk}^{\mathrm{D} 1994 \mathrm{~S}}(\mathrm{n}=28)$.

A possible caveat to this paradigm is that it is $100 \%$ lethal within a relatively short time span, and thus it may not be sensitive enough to observe genotype-specific differences. Therefore, we next infected mice at p3, allowing the mice to develop further (including the brain and the immune system), to allow for increased expression of Lrrk2 which is being induced during these early days; this, to determine whether this would prolong time-to-death and unmask any genotypic differences in survival. Again, Lrrk $2^{\mathrm{D} 1994 \mathrm{~S}}$ mice infected at $\mathrm{p} 3$ did not have any survival differences compared to wild-type or Lrrk $2^{\mathrm{D} 1994 \mathrm{~S}}$ heterozygous mice, and indeed had very similar time-todeath as mice inoculated at p1 (Fig. 3.8B). The earliest death was one mouse at 8dpi (wild-type) with the rest of the mice dying by 12 dpi. The sample sets were: WT $(n=12)$; Lrrk2 ${ }^{\text {D1994S }}$ heterozygous ( $\mathrm{n}=9)$; Lrrk2 ${ }^{\text {D1994S }}(\mathrm{n}=10)$. We saw no differences in survival following $\mathrm{p} 1$ or $\mathrm{p} 3$ inoculation when separated based on sex (Fig. 3.8A, B). 
- WT

- Lrrk2 ${ }^{W T / D S}$

- Lrrk2 ${ }^{\mathrm{DS} / \mathrm{DS}}$

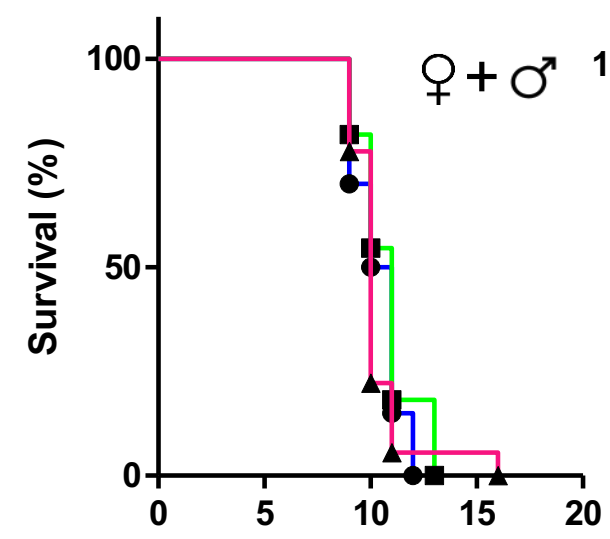

p1 Intracerebral
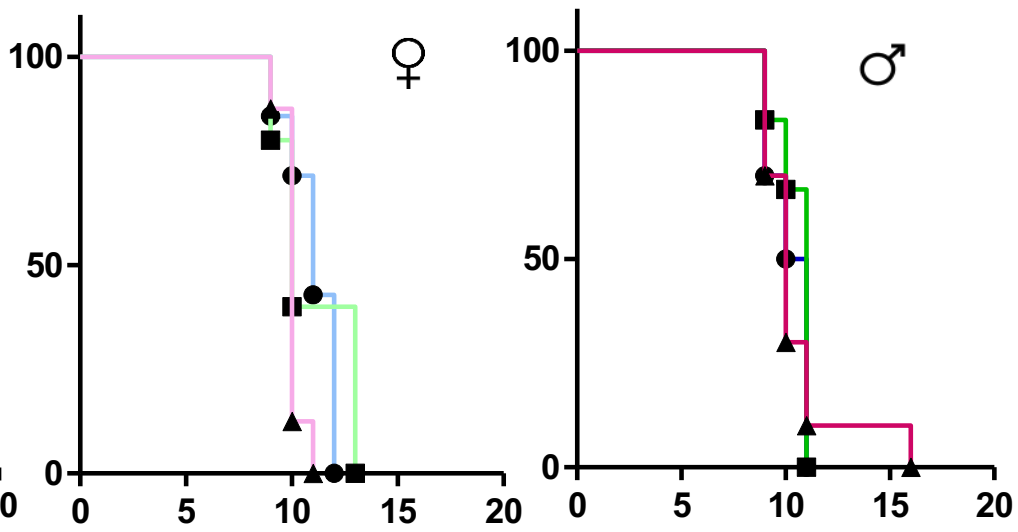

Davs Post Infection

(B)

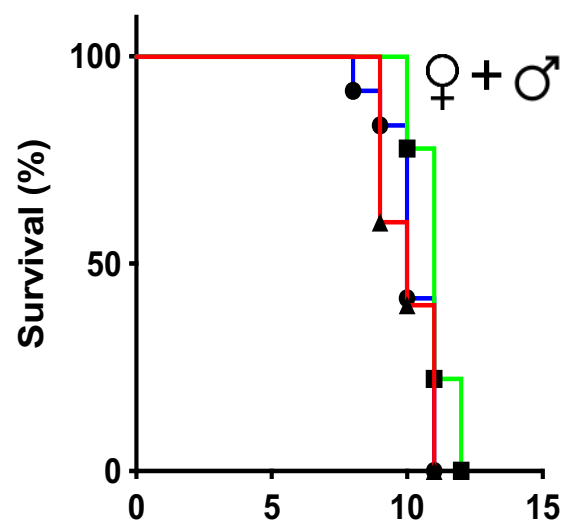

p3 Intracerebral

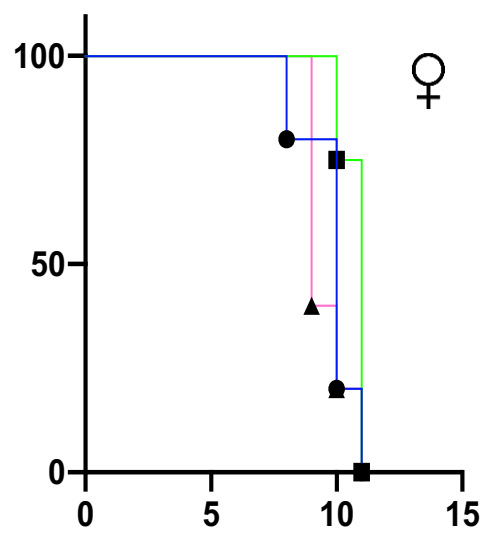

- WT

- Lrrk2WT/DS

- Lrrk2 $2^{\text {DS/DS }}$

Days Post Infection

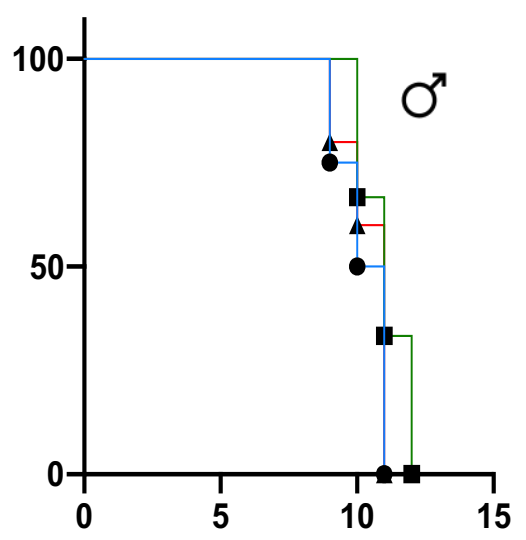

Fig. 3.8: Loss of Lrrk2 kinase activity does not affect survival following an intracerebral injection of reovirus T3D. Newborn mice were infected with $5 \times 10^{2} \mathrm{PFU}$ of reovirus T3D via direct-brain injection to the left hemisphere, and measured for a pre-determined moribund state. Survival was graphically displayed as percent survival against days following infection (dpi). (A) 
Mice inoculated at $\mathrm{p} 1$ had similar survival across genotypes. WT $(\mathrm{n}=20)$; Lrrk2 ${ }^{\text {WT/DS }}(\mathrm{n}=11)$; Lrrk2 ${ }^{1994 \mathrm{~S}}(\mathrm{n}=18)$. (B) Mice intracerebrally-inoculated at $\mathrm{p} 3$ also had similar survival across genotypes. WT $(n=12) ; \operatorname{Lrrk}^{\text {WT/DS }}(n=9) ; \operatorname{Lrrk}^{\text {D1994S }}(n=10)$. No significant differences in survival was found when assessed using a log-rank (Mantel-Cox) test. Note, graphically wild-type mice are denoted WT; Lrrk2 $2^{\text {D1994S }}$ heterozygous mice are denoted Lrrk2 ${ }^{\mathrm{WT} / \mathrm{DS}}$; Lrrk2 ${ }^{\mathrm{D} 1994 \mathrm{~S}}$ homozygous mice are denoted Lrrk2 $2^{\mathrm{DS} / \mathrm{DS}}$. 
A possible explanation for these combined results is that Lrrk2 exerts its primary immune function in the periphery, at least in the context of this reovirus T3D infection model. This will need to be confirmed in future studies using different models, including an adult, direct-brain inoculation paradigm compared to systemic infection paradigms using additional viral models, such as inoculation with vesicular stomatitis virus (VSV) (Drokhlyanksy et al., 2017). Note, adult mice are not susceptible to (peripheral) reovirus T3D-induced encephalitis, which is why we cannot employ our current pathogen model in adults, hence the switch of viral paradigm to VSV.

To explore secondary dissemination of the pathogen (from the brain), we next investigated the corresponding viral titre data in the brain and liver, similar to what was done in the intranasal cohort. First, we looked at 8dpi, to get the best measure of viral titre in the brain before 9dpi, when mice rapidly begin to succumb to the sickness. We measured viral titre in brain, the target organ of infection, and liver, to measure the viral spread from the brain to the periphery. Unexpectedly, given no change in survival following the direct-brain infection, Lrrk2 ${ }^{\text {D1994S }}$ mice seemed to control viral titres in the brain at 8dpi more efficiently than wild-type and heterozygous mice (wildtype vs. Lrrk2 $2^{\mathrm{D} 1994 \mathrm{~S}}$ p-value $=0.0149 ;$ Lrrk2 $^{\mathrm{D} 1994 \mathrm{~S}}$ vs. heterozygous p-value=0.044) (Fig. 3.9A). Lrrk $2^{\text {D1994S }}$ mice had a mean viral titre of $5.93 \times 10^{10} \mathrm{PFU} / \mathrm{g}(\mathrm{n}=16)$, whereas wild-type mice had a mean viral titre of $2.86 \times 10^{11} \mathrm{PFU} / \mathrm{g}(\mathrm{n}=9)$. Note, there were some samples from littermate pairings, however most samples came from homozygous pairs for these PFU analyses.

There were no discernable differences in liver at 8dpi, although, the viral titres in liver were high considering the direct-brain inoculation (wild-type mean liver viral titre: $2.41 \times 10^{6} \mathrm{PFU} / \mathrm{g}$ $(\mathrm{n}=12)$; Lrrk2 ${ }^{\mathrm{D} 1994 \mathrm{~S}}$ mean liver viral titre: $\left.2.74 \times 10^{6} \mathrm{PFU} / \mathrm{g}(\mathrm{n}=16)\right)$ (Fig. 3.9A), which we attribute to the damage done to the blood brain barrier by the needle tract. Again, consistent with this dataset 
thus far, this significance in viral titre in the brain appeared to be co-regulated by female sex (Fig. $3.9 \mathrm{~B}, \mathrm{C})$.

As we observed viral titre differences between genotypes specifically at early points of disease in our intranasal paradigm (see above), we next measured viral titre in brain and liver of mice collected at 3dpi following intracerebral reovirus infection. There were no significant differences in the brain or liver between Lrrk2 $2^{\text {D1994S }}$ homozygous or heterozygous, or wild-type mice at 3dpi (Fig. 3.10). Mean viral titres in liver at 3dpi for wild-type, heterozygous, and Lrrk2 $2^{\text {D1994S }}$ mice were $1.03 \times 10^{4} \mathrm{PFU} / \mathrm{g}(\mathrm{n}=3), 1.07 \times 10^{5} \mathrm{PFU} / \mathrm{g}(\mathrm{n}=8)$, and $2.5 \times 10^{3} \mathrm{PFU} / \mathrm{g}(\mathrm{n}=4)$, respectively. Mean viral titres in brain at 3dpi for wild-type, heterozygous, and Lrrk $2^{\mathrm{D} 1994 \mathrm{~S}}$ mice were $7.56 \times 10^{5} \mathrm{PFU} / \mathrm{g}(\mathrm{n}=4), 1.87 \times 10^{6} \mathrm{PFU} / \mathrm{g}(\mathrm{n}=7)$, and $1.09 \times 10^{6} \mathrm{PFU} / \mathrm{g}(\mathrm{n}=5)$, respectively. However, a larger sample set and adequate representation for males and females needs to be increased before final conclusions can be drawn from these viral titre experiments following intracerebral inoculation. 
(A)
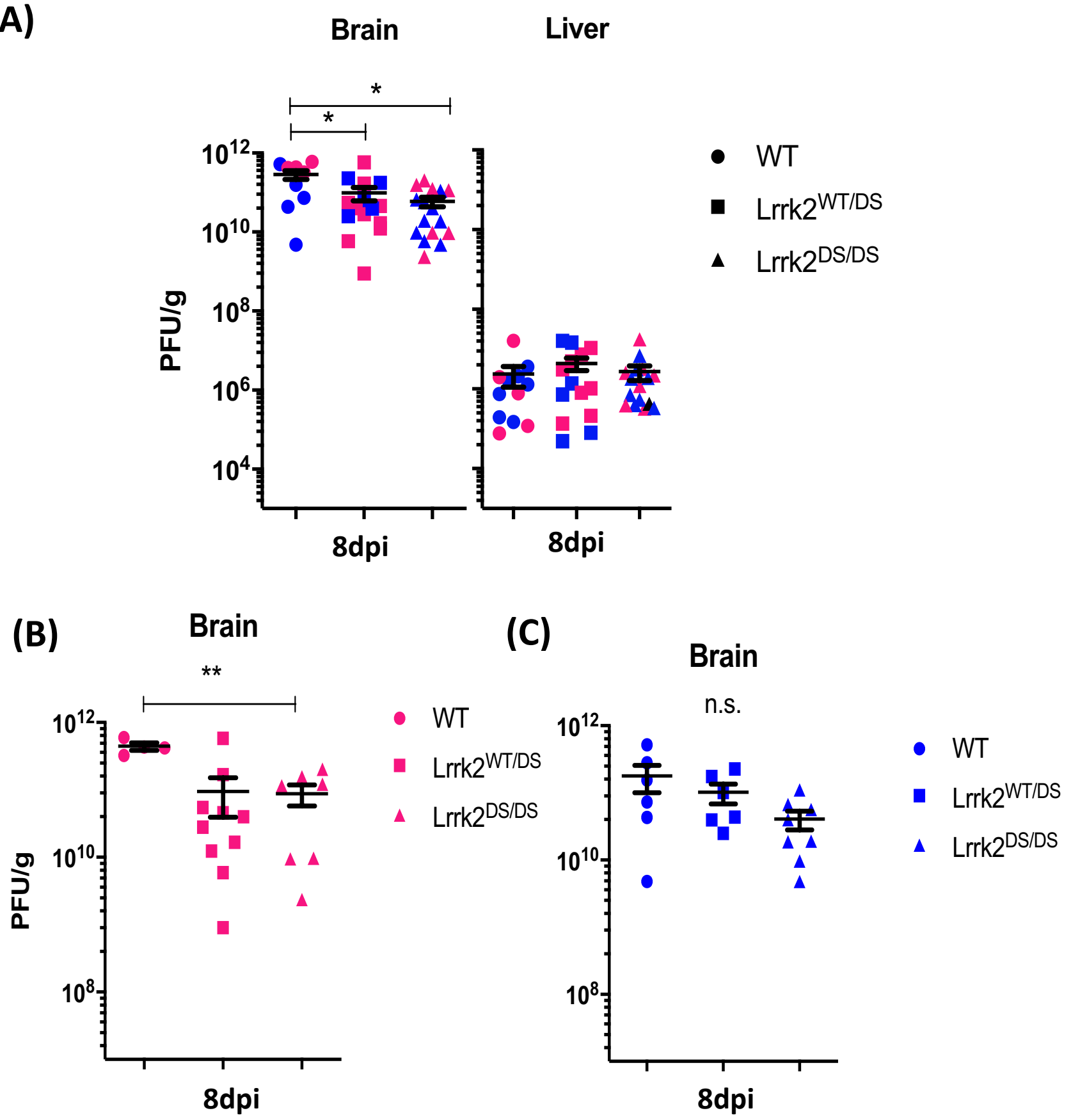

Fig. 3.9: Loss of Lrrk2 kinase activity causes an unexpected decrease in viral titres with a female sex bias, following an intracerebral injection of reovirus T3D. Newborn mice were infected with $5 \times 10^{2} \mathrm{PFU}$ of reovirus T3D via direct-brain injection to the left hemisphere, and measured for a pre-determined moribund state. Lrrk2 ${ }^{\mathrm{D} 1994 \mathrm{~S}}$ homozygous and heterozygous mice, 
and wild-type mice were sacrificed at 8dpi (A) and liver and brain were collected and homogenized. Data are represented in PFU/g, where each symbol represents one animal. Pink data points indicate females, and blue data points indicate males. Sample sets for 8dpi liver are: WT $(\mathrm{n}=12)$; Lrrk2 ${ }^{\text {D1994S }}$ heterozygous $(n=14)$; Lrrk2 ${ }^{\text {D1994S }}(n=16)$; and for brain are: WT $(n=9)$; Lrrk2 ${ }^{\text {D1994S }}$ heterozygous $(n=16) ; \operatorname{Lrrk}^{\text {D1994S }}(n=16)$. The $p$-value between wild-type and heterozygous $=0.0445$. The $p$-value between wild-type and Lrrk $2^{\mathrm{D} 1994 \mathrm{~S}}=0.0149$. Data for $8 \mathrm{dpi} \mathrm{PFU}$ in brains were separated by sex, female (A) and male (B). The p-value between female wild-type and $\operatorname{Lrrk} 2^{\mathrm{D} 1994 \mathrm{~S}}$ brains $=0.0061$. The $\mathrm{p}$-value between male wild-type and $\operatorname{Lrrk} 2^{\mathrm{D} 1994 \mathrm{~S}}$ brains $=0.1209$ (not significant). ${ }^{*} \mathrm{P} \leq 0.05,{ }^{*} \mathrm{P} \leq 0.01$. Test used for PFU data: non-parametric Mann-Whitney test. Error bars represent mean \pm SEM. Note, graphically wild-type mice are denoted WT; Lrrk2 ${ }^{\text {D1994S }}$ heterozygous mice are denoted Lrrk2 ${ }^{\text {WT/DS}}$; Lrrk2 ${ }^{\text {D1994S }}$ homozygous mice are denoted Lrrk2 $2^{\mathrm{DS} / \mathrm{DS}}$. 


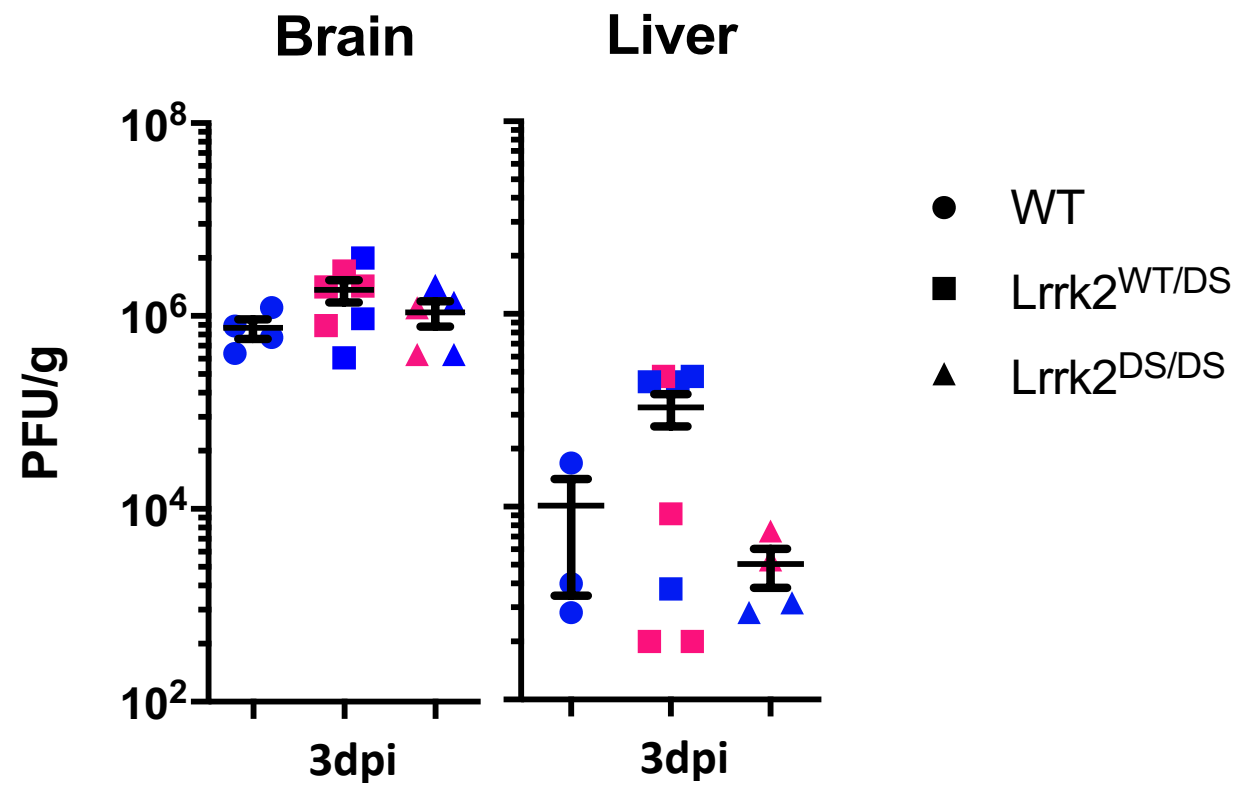

Fig. 3.10: Loss of Lrrk2 kinase activity does not affect viral titres at 3 days post infection following an intracerebral viral injection compared to wild-type mice. Newborn mice were infected with $5 \times 10^{2}$ PFU of reovirus T3D via direct-brain injection to the left hemisphere and measured for a pre-determined moribund state. Lrrk2 $2^{\mathrm{D} 1994 \mathrm{~S}}$ homozygous and heterozygous mice, and wild-type mice were sacrificed at 3dpi and liver and brain were collected and homogenized. Data are represented in PFU/g, where each symbol represents one animal. Pink data points indicate females, and blue data points indicate males. Sample sets for 3dpi liver are: WT ( $n=3)$; Lrrk2 ${ }^{\text {D1994S }}$ heterozygous $(n=8)$; Lrrk2 ${ }^{\text {D1994S }}(n=4)$; and for brain are: WT $(n=4)$; Lrrk2 ${ }^{\text {D1994S }}$ heterozygous $(n=7)$; Lrrk2 ${ }^{\text {D1994S }}(n=5)$. Test used for PFU data: non-parametric Mann-Whitney test. Error bars represent mean \pm SEM. Note, graphically wild-type mice are denoted WT; Lrrk2 ${ }^{\text {D1994S }}$ heterozygous mice are denoted Lrrk2 ${ }^{\mathrm{WT} / \mathrm{DS}}$; Lrrk $2^{\mathrm{D} 1994 \mathrm{~S}}$ homozygous mice are denoted Lrrk2 ${ }^{\mathrm{DS} / \mathrm{DS}}$. 


\section{Lrrk2 $2^{G 2019 S}$ mice have equal survival rates as heterozygous and wild-type mice following reovirus T3D direct-brain inoculation.}

To further investigate the role of Lrrk2 and variants of the protein in response to infection in the brain $v s$. the periphery, we next investigated Lrrk2 $2^{\mathrm{G} 2019 \mathrm{~S}}$ mice. In a preliminary cohort (wild-type $\mathrm{n}=5$; Lrrk2 ${ }^{\mathrm{G} 2019 \mathrm{~S}}$ heterozygous $\mathrm{n}=6$; $\operatorname{Lrrk} 2^{\mathrm{G} 2019 \mathrm{~S}} \mathrm{n}=6$ ), we found that similarly to Lrrk2 kinase-dead mice, mice carrying a p.G2019S mutation did not have increased or decreased time-to-death responses to a direct-brain inoculation of reovirus T3D compared to wild-type mice (Fig. 3.11). Specifically, mice began to die on $8 \mathrm{dpi}$ and all mice were dead by $12 \mathrm{dpi}$. These results were interesting, as we have noted that female Lrrk2 $2^{\mathrm{G} 2019 \mathrm{~S}}$ mice had a survival deficit in response to nasally inoculated reovirus T3D (Fig. 1.3). Importantly, this current intracerebral inoculation cohort of Lrrk2 $2^{\mathrm{G} 2019 \mathrm{~S}}$ mice was very small, and the sample set must be greatly increased to probe further for any differences. Indeed, in the intranasal inoculation p.G2019S data, we only found significant survival differences in females, therefore we must also increase the sample size to have adequate survival curves for both males and females individually. This work is ongoing, as is the collection of brain and liver specimens from Lrrk $2^{\mathrm{G} 2019 \mathrm{~S}}$ mice and wild-type littermates to measure viral titre following intracerebral inoculation in these mice. 


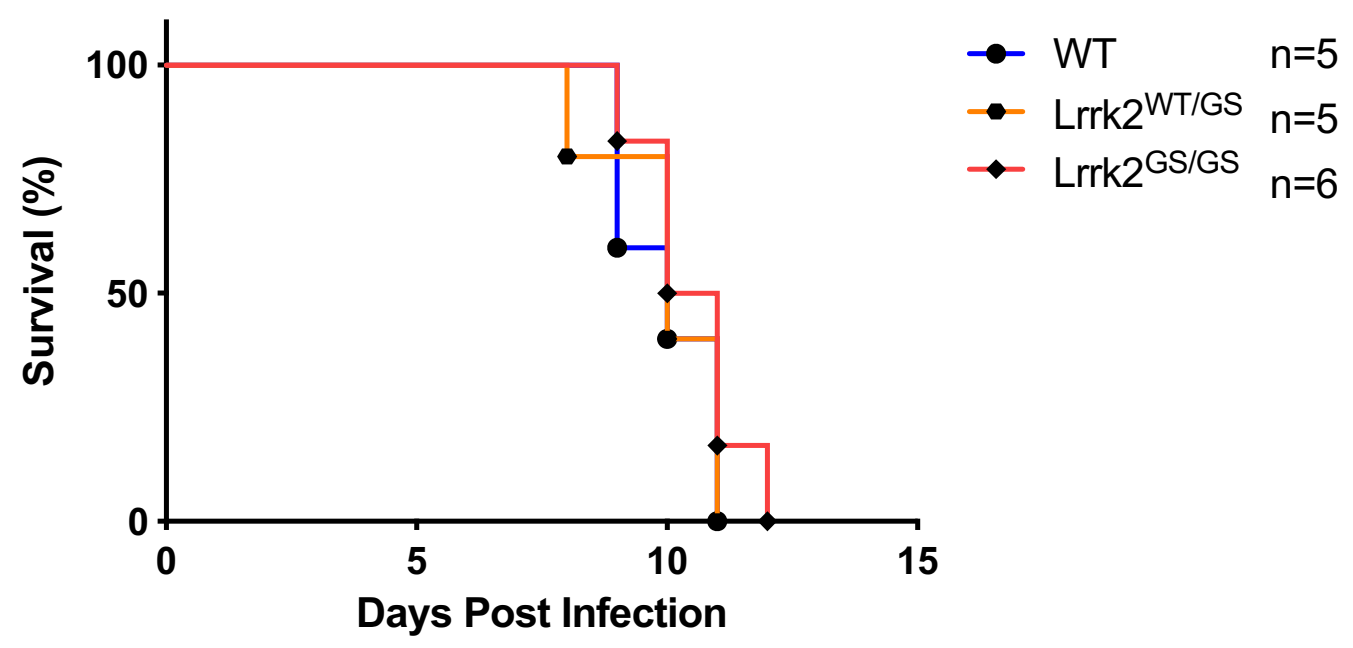

Fig. 3.11: Lrrk2 ${ }^{\mathrm{G} 2019 \mathrm{~S}}$ mice have equal survival rates as heterozygous and wild-type mice following an intracerebral reovirus T3D inoculation. Newborn mice were infected with $5 \times 10^{2}$ PFU of reovirus T3D via direct-brain injection to the left hemisphere and measured for a predetermined moribund state. Survival was graphically displayed as percent survival against days following infection (dpi). Sample set n-values are indicated on the graph. Test used: log rank (Mantel-Cox) test. 
In response to a systemic bacterial infection, Lrrk2 kinase-dead mice behave the same as wildtype mice in terms of bacterial burden in spleen and survival rate.

Our team had also employed a second infection paradigm with a different pathogen to further inform the viral inoculation results. In this paradigm, adult mice (8-12 weeks old) were inoculated intravenously with Salmonella typhimurium at a dose of $200 \mathrm{CFU}$ (Fig. 2.1C). In C57BL/6mice, Salmonella causes fatal sepsis which will indefinitely kill all mice (Shutinoski et al., 2016). When measuring outcomes in Lrrk2 $2^{\mathrm{G} 2019 \mathrm{~S}}$ mice, this mutation was protective; leading to higher survival and lower bacterial titres (CFU) compared to wild-type mice (Shutinoski et al., 2019). In contrast, Lrrk2 KO was detrimental, with higher bacterial titres counted in the spleen, though no change in survival was recorded compared to wild-type mice (Shutinoski et al., 2019).

To determine whether kinase activity was required in this outcome, these experiments were repeated using the kinase-dead Lrrk $2^{\text {D1994S }}$ mice. Two outcome measures were assessed: survival (measured as time-to-death) and CFU (done by Dr. B. Shutinoski in our team). Interestingly, when looking at females carrying the kinase-dead Lrrk $2^{\text {D1994S }}$ mutation, the survival curve of these mice was the same as wild-type mice (wild-type $n=6$; Lrrk2 ${ }^{\text {D1994S }}$ heterozygous $n=10$; $\operatorname{Lrrk}^{\text {D1994S }} n=10$ ), with mice beginning to die at $7 \mathrm{dpi}$, and the last mouse dying at $19 \mathrm{dpi}$. Most mice succumbed to the sickness on 8, 9, and 10dpi (Fig. 3.12A). However, with Lrrk2 kinase-dead mice, there was no difference in bacterial counts (Fig. 3.12B). Wild-type mice had a mean of $1.37 \times 10^{6} \mathrm{CFU}$ in the spleen at $5 \mathrm{dpi}$, heterozygous mice had a mean $1.95 \times 10^{6} \mathrm{CFU}$, and Lrrk $2^{\mathrm{D} 1994 \mathrm{~S}}$ had a mean $1.13 \times 10^{6}$ CFU. Of note, not all mice were littermates (though some were), as all came from the same colony bred on the same background. Each genotype had $\mathrm{n}=5$ for $\mathrm{CFU}$ analysis.

These results suggested that the lack of Lrrk2 kinase activity did not confer a protective or harmful effect in response to this bacterial sepsis paradigm, and did not cause a difference in 
bacterial titres. This reaffirmed our previous findings, in which Lrrk2 kinase activity was not seen as essential for the host's immune response, and secondly, that wild-type Lrrk2 kinase activity does not seem to be important in bacterial clearance, although the protein itself is. Nevertheless, the p.G2019S mutant conferred an independent, beneficial gain-of-function effect, in the sepsis paradigm. Moreover, in this model we did not see a difference in survival in the Lrrk2 knockout mice compared to wild-type mice, which could suggest that function of Lrrk2 in the immune system is pathogen-dependent, and may not be as important in response to bacterial threats as we had seen with a viral threat. 

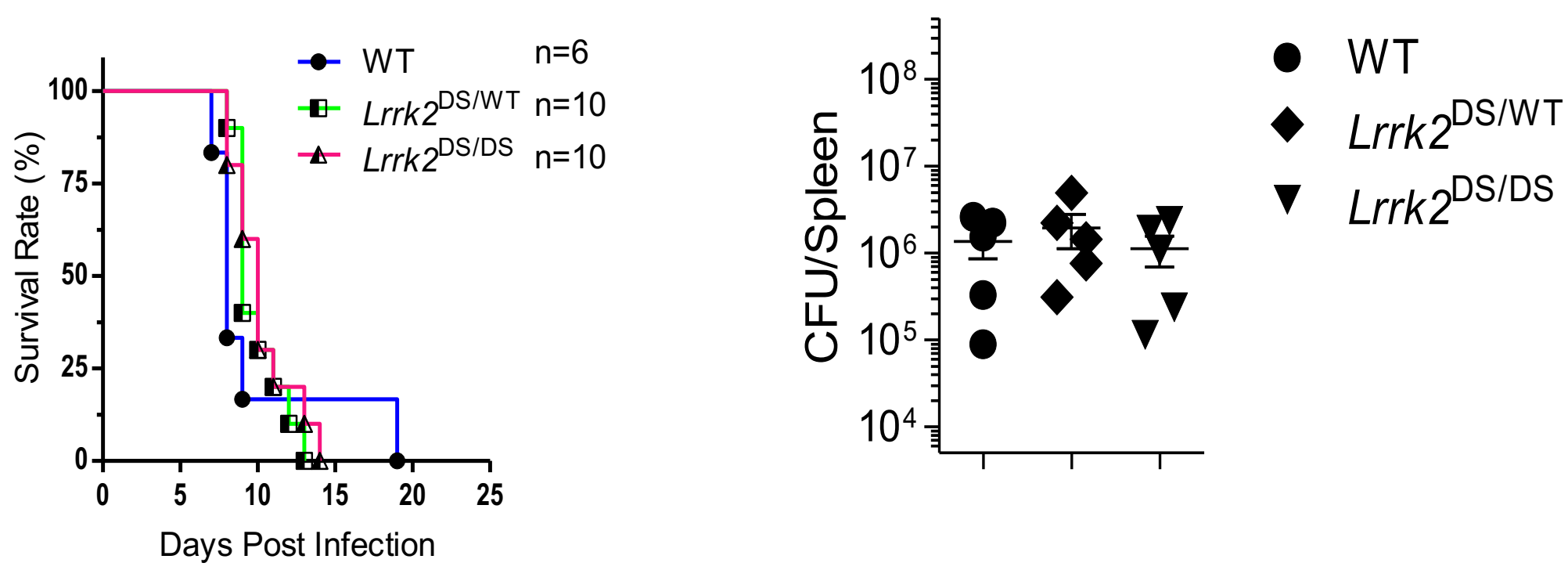

Fig. 3.12: Loss of Lrrk2 kinase activity does not affect the survival or bacterial burden in murine spleens in response to Salmonella typhimurium infection. Adult mice were infected intravenously with 200 CFU of Salmonella typhimurium. (A) Survival rate (time-to-death) was measured in homozygous and heterozygous Lrrk2 ${ }^{\text {D1994S }}$ and wild-type mice. Sample set n-values are indicated on the graph. (B) CFU (Colony forming units) counts of Salmonella infected spleen at day 5 post infection from wild-type, heterozygous, and Lrrk2 ${ }^{\mathrm{D} 1994 \mathrm{~S}}$ mice are graphically displayed. Each CFU data point represents one animal ( $\mathrm{n}=5$ for each genotype). Survival statistical analysis was done using the log-rank test. CFU analysis was statistically tested using MannWhitney test. Error bars represent mean \pm SEM. 


\section{In vitro, Lrrk2 kinase-dead-derived macrophages have decreased immune signaling as indicated by pSTAT activation.}

To investigate the intriguing Lrrk2 kinase-mediated effects observed in vivo, we used bone marrow-derived macrophages to examine the effects of Lrrk2 kinase activity in vitro to begin to explore the underlying molecular mechanisms. We derived macrophages from the bone marrow of Lrrk2 ${ }^{\text {D1994S }}$ mice and wild-type littermates. Bone-marrow derived macrophages (BMDMs) were treated with reovirus at a MOI 3 for one or two hours before being lysed for Western blot analysis. We then probed for phosphorylated-STAT (pSTAT) and total STAT (tSTAT) levels in wild-type and Lrrk $2^{\text {D1994S }}$-derived cell samples $(n=3)$. The fold change of pSTAT/tSTAT was used as a readout to measure an active immune signaling pathway (i.e., the JAK-STAT pathway activated by upstream chemokines (Rauch et al., 2013)). In this experiment, all cell culture work, treatment, and protein collection were performed by M. Lunn, however readouts (Western blot analysis and densitometry) were performed by Quinton Hake-Volling in our lab.

Previous findings in the lab had shown decreased pSTAT/tSTAT signaling in Lrrk2 KO BMDMs, and conversely increased signaling in Lrrk2 ${ }^{\mathrm{G} 2019 \mathrm{~S}}$ BMDMs. In the present study, we observed the expected, infection-dependent increase in pSTAT/tSTAT signal; however, when comparing wild-type and Lrrk2 $2^{\text {D1994S }}$ BMDMs at both timepoints following infection, there seems to be a trend toward Lrrk2 ${ }^{\text {D1994S }}$ BMDMs having a lower pSTAT/tSTAT ratio, which suggests a decreased immune signaling by the mutant in response to the viral infection, at least ex vivo (Fig. 3.13A, B). This finding complements the in vivo data that the Lrrk2 kinase-dead mutation confers a lesser immune response, and the Lrrk2 ${ }^{\mathrm{G} 2019 \mathrm{~S}}$ mutation augments it; collectively, these findings suggest to us that Lrrk2 modulates the degree of the immune response in an infected host - with the Lrrk $2^{\mathrm{G} 2019 \mathrm{~S}}$ having an increased response and the Lrrk2 ${ }^{\mathrm{D} 1994 \mathrm{~S}}$ having a dampened response. 
(A)

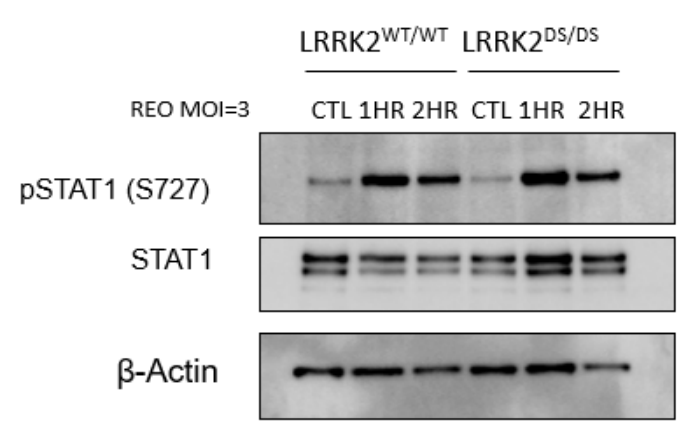

(B)

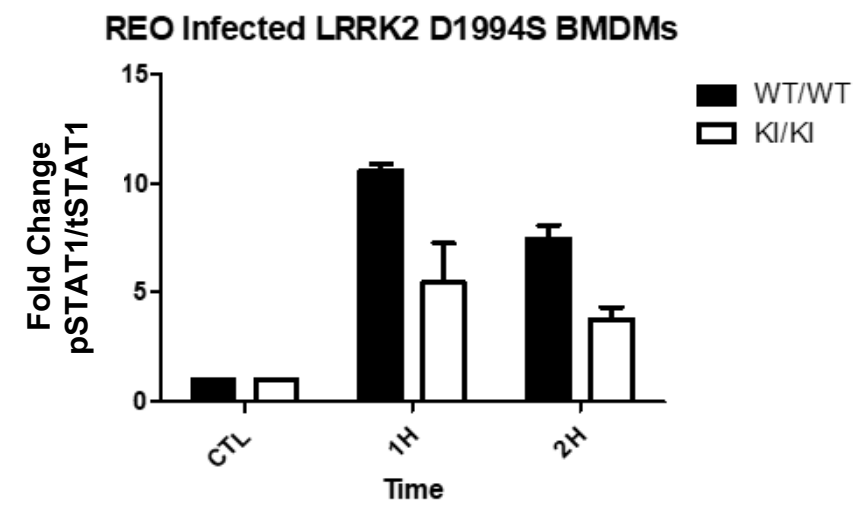

Fig. 3.13: Genetically ablating Lrrk2 kinase activity causes a decrease in the pSTAT/tSTAT ratio, indicating a decrease in the activation of immune signalling. (A) Western blot of antiSTAT1 and anti-pSTAT1 and (B) correlating relative fold change measured by densitometry analysis $(n=3)$. Note, graphically wild-type mice are denoted WT/WT; Lrrk2 ${ }^{\text {D1994S }}$ homozygous mice are denoted $\mathrm{KI} / \mathrm{KI}$. 


\section{Treatment with a selective Lrrk2 kinase inhibitor, MLi-2, in bone marrow-derived macrophages to examine cell viability and determine viral load following infection.}

Next, we sought to investigate whether the results seen in vivo by genetically inhibiting Lrrk2 kinase activity could be reproduced ex vivo using a pharmacological Lrrk2 kinase inhibitor. We employed MLi-2, a published and validated Lrrk2 kinase inhibitor (Fell et al., 2015). Wildtype BMDMs were grown and seeded in duplicate for cell viability and plaque assay analysis. One subset of plates were treated with PBS control and one set were treated with 100nM of MLi-2 for two hours prior to treatment and for the entirety of the infection to inhibit Lrrk2 kinase activity, a dose previously demonstrated to be effective (Fan et al., 2018). For cell viability, cells were then treated with reovirus T3D for 24 hours at varying MOIs. Interestingly, we found no difference between treated and untreated cells in terms of cell viability $(n=4)$ (Fig. 3.14A). Both treated and untreated cells had only about $20 \%$ death rates.

When measuring plaque forming units, BMDMs were infected with reovirus T3D at a MOI of 5 for 24 hours and the cell lysates were used to infect L929 cells in a standard plaque assay protocol. Again, there was no difference in viral load between MLi-2 treated and untreated cells ( $\mathrm{n}=2$ for each) (Fig. 3.14B). Untreated cells had a mean viral titre of 3.97x $10^{4} \mathrm{PFU} / \mathrm{mL}$ and MLi2-inhibited cells had a mean viral titre of $2.54 \times 10^{4} \mathrm{PFU} / \mathrm{mL}$.

Taken together, these results suggest that inhibiting Lrrk2 kinase activity pharmacologically in wild-type BMDMs does not cause a difference in the infection susceptibility phenotype of these cells - suggesting that Lrrk2 kinase activity is not essential in responding to viral infection ex vivo using a primary, bone-marrow-derived immune cell as a model.

Future directions include investigating the cytokine and chemokine profile of these macrophages derived from Lrrk2 ${ }^{\mathrm{D} 1994 \mathrm{~S}}$ mice following a viral infection both at the mRNA (by 
qPCR), and protein (by ELISA) expression levels. Additionally, it would be interesting to measure the response to infection in vivo (as seen in paradigms (Fig. 21.A, B)) in wild-type mice treated or untreated with the selective Lrrk2 kinase inhibitor, MLi-2. We have demonstrated the role of Lrrk2 kinase activity in the immune system when genetically knocking out this function; however, it would be insightful to see if these results are consistent when Lrrk2 kinase activity is inhibited pharmacologically. It is particularly relevant as it is the pharmacologically inhibition of LRRK2 kinase activity that is currently in human trials. 
(A)

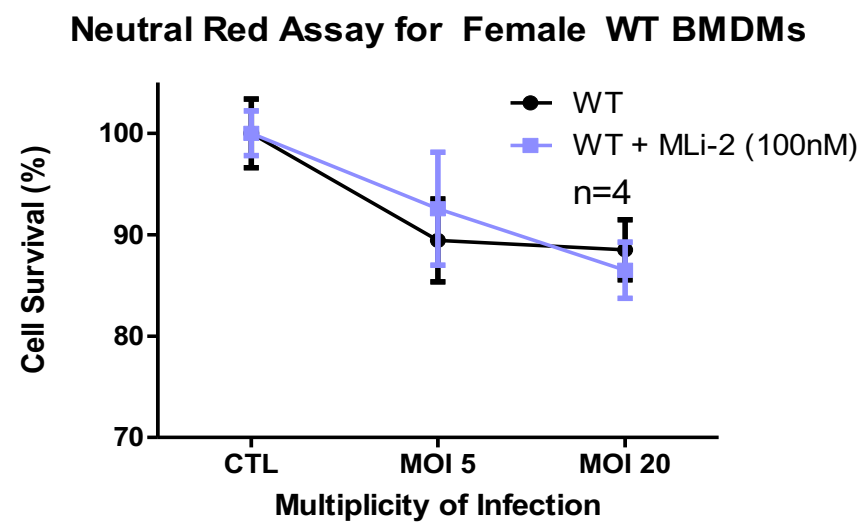

(B)

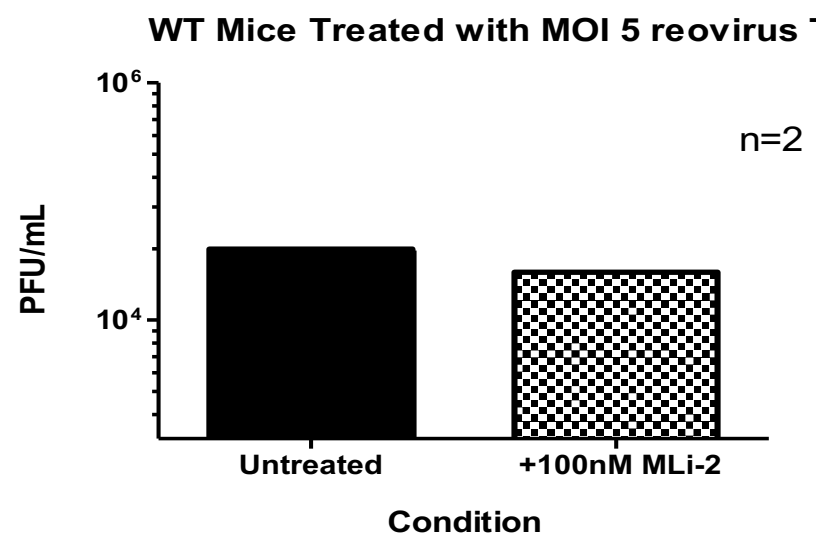

Fig. 3.14: Pharmacologically inhibiting Lrrk2 kinase activity does not affect cell viability or viral burden in cells. (A) Wild-type BMDMs treated with the selective Lrrk2 kinase inhibitor, MLi2 $(100 \mu \mathrm{M})$ or with PBS vehicle control were infected with MOI 5 or MOI 20 reovirus T3D for 24 hours and cell viability (via neutral red assay) was measured. (B) Wild-type BMDMs treated with MLi2 $(100 \mu \mathrm{M})$ or with PBS vehicle control were infected with MOI 5 reovirus T3D for 24 hours and cells were lysed for viral titre sample. Cell lysis was used to infect L929 cells and measure PFU/mL (via plaque assay). 


\section{Discussion}

This thesis sought to elucidate the role of LRRK2 kinase activity in innate immune functions and its effects in both the brain-specific and peripheral immune responses. I originally hypothesized that LRRK2 kinase activity was important in its role in fighting microbial infections, and that genetically or pharmacologically ablating its kinase activity would reverse any protective benefits of wild-type LRRK2 in protecting against these infections. Through several infection paradigms and Lrrk2-variant mouse models, I have successfully disproved this hypothesis and uncovered multiple important insights into physiological and pathological LRRK2 function, all of which are possibly relevant to the pathogenesis of PD (and by inference, some of which are relevant to other $L R R K 2$-linked disorders).

Through ongoing work, our team has clearly implicated LRRK2 in the immune system. Specifically, I have indicated five main things relevant to mammalian LRRK2 biology: (i) wildtype Lrrk2 functions in the immune system independent upon its kinase activity; (ii) Lrrk2 behaves with a female sex bias; (iii) Lrrk2 acts in a pathogen- and organ- dependent manner; (iv) Lrrk2 ${ }^{\mathrm{G} 2019 \mathrm{~S}}$ confers an independent gain-of-function type effect in vivo, and (v) Lrrk2 seems to work predominantly in the periphery rather than the brain when protecting the nervous system from a systemic infection.

\subsection{LRRK2 in Immune Function}

Evidence supporting LRRK2 in immune function has been steadily growing. Some of the first data initiating this came from our lab, demonstrating that LRRK2 is highly expressed in cells of the innate immune system when compared to neurons (Hakimi et al., 2011). Importantly, LRRK2 is more highly expressed in the periphery than the brain in general, specifically in the 
lungs and kidneys (Hakimi et al., 2011; Shutinoski et al., 2019). We have demonstrated thoroughly that variants in LRRK2 have the ability to modulate infection, both in the context of a bacterial infection in the periphery (i.e., outside the CNS) and a viral, systemic infection that includes the brain.

Salmonella typhimurium causes sepsis in $\mathrm{C} 57 \mathrm{Bl} / 6$ mice, which is fatal. This paradigm was used to test the role of Lrrk2 in the context of adult infection with a live, replicating microbe. Unexpectedly, Lrrk2 knockout (KO) mice did not have decreased survival in response to this infection paradigm compared to wild-type mice; however, they were also not protected (Shutinoski et al., 2019). Lrrk2 KO mice did show a lesser control over bacterial replication, as indicated by the increased bacterial burden in the spleen by 5 dpi (Shutinoski et al., 2019).

When this same paradigm was employed on Lrrk2 $2^{\mathrm{G} 2019 \mathrm{~S}}$ mice, we found interesting results. Lrrk2 ${ }^{\mathrm{G} 2019 \mathrm{~S}}$ mice displayed a pronounced protective phenotype in response to Salmonella typhimurium, when measuring both survival (indicated by time-to-death) and bacterial burden (Shutinoski et al., 2019). Both Lrrk2 ${ }^{\mathrm{G} 2019 \mathrm{~S}}$ homozygous and heterozygous mice had significantly longer time-to-death than wild-type mice, suggesting that even one mutant Lrrk2 allele was protective in this paradigm. This was also demonstrated in bacterial burden, where both Lrrk2 ${ }^{\mathrm{G} 2019 \mathrm{~S}}$ mice and heterozygous mice had significantly less bacterial burden in the spleen at 5dpi than wildtype mice, specifically in females (Shutinoski et al., 2019). See Section 4.5 for a more in depth examination on the sex bias seen with Lrrk2.

The protective phenotype seen with the p.G2019S mutation likely helps to explain why this mutation has persisted over generations and has arisen independently in human evolution on three separate occasions (Lesage et al., 2010). These data would suggest that the p.G2019S mutation is evolutionarily advantageous in response to microbial infections (referred to as a 
'heterozygous advantage' in biology), i.e., it may pose a protective benefit to the carrier. The pathogenesis of this mutation may exhibit its effect later in life, coinciding with the fact that age is the greatest risk factor in PD (Schlossmacher et al., 2017).

These data are consistent with the work of Liu et al. (2017) in which they employed a Salmonella typhimurium paradigm similar to ours. They also demonstrated that Lrrk2 KO animals had increased bacterial burden in the spleen, as well as in blood and in peritoneal fluid (they administered the infection via IP injection). Of note, they did observe a survival deficit in the Lrrk2 KO mice in their paradigm, while our lab did not. Next, they indicated decreased bacterial burden in spleen, blood, and peritoneal fluid of Lrrk $2^{\mathrm{G} 2019 \mathrm{~S}}$ mice. They did not measure survival in Lrrk2 ${ }^{\mathrm{G} 2019 \mathrm{~S}}$ mice. They attribute these observed changes in bacterial burden mainly to NLRC4 inflammasome activation and downstream IL-1 $\beta$ production. In vitro, using BMDMs, they demonstrated that Lrrk2 phosphorylated Ser533 on NCLRC4, leading to activation of the NCLRC4 inflammasome (Liu et al., 2017). This activation is associated with downstream signaling of pro-inflammatory cytokines such as IL-1 $\beta$ (Franchi et al., 2006). Consistent with this, they demonstrated in vivo that Lrrk2 KO mice had decreased IL-1 $\beta$ levels, whereas Lrrk2 ${ }^{\text {G2019S }}$ mice had increased levels (Liu et al., 2017).

This is suggestive of an overall pro-inflammatory profile associated with the p.G2019S mutation. Indeed, IL-1 $1 \beta$ levels have been used to successfully differentiate asymptomatic human p.G2019S carriers from non-carriers, where higher levels of IL-1 $\beta$ was also correlated with a greater risk for PD in the carriers (Dzamko et al., 2016).

In our LRRK2 research, the lab next wished to employ an infection paradigm that would have a direct impact on brain health. Therefore, we utilized reovirus T3D to nasally infect suckling pups. This model system had been developed by Dr. Earl Brown, professor emeritus of molecular 
virology at uOttawa, and a part-time member of the lab, who mentors trainees in carrying out inoculation experiments. In suckling pups (p1), reovirus T3D has tropism for the brain and causes encephalitis which can be fatal (Gauvin et al., 2013). Note, mice infected older than p10 do not succumb to the infection (encephalitis) with this paradigm. Secondly, by using pups, we looked directly at the innate immune response, as pups do not begin developing an adaptive immune response until a minimum of nine days after birth, and do not have a fully mature adaptive immune system until four weeks of age (Landreth, 2002).

In this paradigm, when infecting Lrrk2 KO mice, our lab found a survival deficit in mice lacking Lrrk2 (Shutinoski et al., 2019). This was not associated with a difference in viral burden; however, the sample set remains small in this experiment, and it is very possible a difference may be identified if the sample set is increased. Based on previous experiments, statistical significance tends to be demonstrated with sample sets around $\mathrm{n} \geq 10$.

When examining Lrrk $2^{\mathrm{G} 2019 \mathrm{~S}}$ mice, again better control of viral replication was found, consistent with the bacterial infection model. Lrrk $2^{\mathrm{G} 2019 \mathrm{~S}}$ mice had decreased viral burden in the lung at peak viral titre of this organ, $3 \mathrm{dpi}$, and decreased viral burden in the brain at its peak viral titre day, 11dpi compared to wild-type mice. However, paradoxically, this decreased viral burden associated with Lrrk2 $2^{\mathrm{G} 2019 \mathrm{~S}}$ mice was also associated with decreased (not improved) survival from brain infection (Shutinoski et al., 2019). Therefore, these mice cleared the virus more efficiently but also had increased mortality, in the females specifically. We hypothesize that Lrrk2 ${ }^{\mathrm{G} 2019 \mathrm{~S}}$ mice show an overshooting immune response; therefore, they are mounting an immune response that is strong enough to efficiently clear the bacteria, but is 'excessive' (when compared to wild-type animals) and associated with detriment to the host. Indeed, members of our lab have reported increased myeloid cell recruitment in reovirus-infected brains (measured via flow cytometry) and 
increased chemotactic signalling in reovirus-infected BMDMs of Lrrk2 ${ }^{\mathrm{G} 2019 \mathrm{~S}}$ animals (Shutinoski et al., 2019). This is consistent with the reports of Liu et al. (2017) and others suggesting a proinflammatory phenotype associated with the p.G2019S mutation.

In accordance, Moehle et al. (2015) also reported an exaggerated chemotactic response associated with the p.G2019S mutation. In rats expressing Lrrk2 p.G2019S, they found increased myeloid cell infiltration to the point of injection of LPS in the substantia nigra. Additionally, they found enhanced chemotaxis in myeloid cells that expressed Lrrk2 p.G2019S in vitro. When they treated these p.G2019S-expressing cells with two selective Lrrk2 kinase inhibitors in vitro, they found that the enhanced chemotaxis was reversed, and these cells began to behave like wild-type cells. They posit that this increased myeloid cell chemotaxis and mobility is due to Lrrk2's ability to bind with actin-regulatory proteins and affect cytoskeleton remodelling. They show evidence that the p.G2019S mutation increases binding and this interaction, possibly leading to enhanced myeloid cell mobility in p.G2019S carriers (Moehle et al., 2015).

This initial data set demonstrated that LRRK2 plays a role in immune response. Secondly, they suggested the kinase activity of this protein was important in the immune response, as seen by the ability of the p.G2019S mutation (i.e., increased kinase activity) to modulate host response to infection. Therefore, our next research question was: What is the contribution of Lrrk2 kinase activity in the host's immune response?

\subsection{LRRK2 Kinase Activity in Immune Function}

To investigate the role of Lrrk2 kinase activity, we used genetically engineered, Lrrk2 kinase-dead mice. These mice carry a knock-in p.D1994S mutation in the murine Lrrk2 protein, and were created, and first characterized by Herzig et al. (2011). This mutation renders these mice 
kinase-dead, while also affecting the stability of the protein, as Herzig et al. (2011) have noted that the protein is about half as abundant in the Lrrk $2^{\mathrm{D} 1994 \mathrm{~S}}$ mice compared to wild-type. We repeated these experiments and confirmed these mice are kinase-dead, as measured by Lrrk 2 pSer935 levels. Additionally, in adult mice, we found much lower abundance of Lrrk2 in the brain compared to wild-type mice, but similar levels of Lrrk2 in peripheral organs such as lungs and spleen when comparing wild-type and kinase-dead mice. We also confirmed this in infected organs (3dpi and 11dpi) used in the reovirus T3D paradigm (Fig. 3.3). Through observation, we did not notice any phenotypic differences (i.e., darkened colour or increased size) in spleens obtained from wild-type or kinase-dead mice at this young age (p3, p11), as splenic phenotypic differences have been reported in adult kinase-dead mice (Herzig et al., 2011).

We first employed the bacterial Salmonella typhimurium infection paradigm in Lrrk2 ${ }^{\text {D1994S }}$ kinase-dead mice. Similar to Lrrk2 KO mice, we found no differences in time-to-death between wild-type, heterozygous, or kinase-dead mice (Fig. 3.12A). As absence of the Lrrk2 protein did not play a role in time-to-death following Salmonella infection, we did not expect the Lrrk2 kinasedead mice to affect survival. Next, we measured bacterial burden and found no differences in bacterial burden in the spleen following Salmonella infection in these mice. This was surprising, as the Lrrk2 KO mice did have increased bacterial burden. These data suggests that the Lrrk2 protein per se, but not its phosphorylative (kinase) function, is important in bacterial clearance in this paradigm. Importantly, these data also suggested that the protective phenotype associated with the hyperactive kinase p.G2019S mutation was indeed a net gain-of-function (See Section 4.3).

Interestingly, in the same paper discussed earlier, Liu et al. (2017) tested a Lrrk2 kinasedead model in their paradigm. Instead of genetic ablation, they utilized the LRRK2 kinase inhibitor GSK2578215A to pharmacologically ablate the kinase activity in vivo. They pretreated wild-type 
animals with this inhibitor prior to infection. Interestingly, they found that mice treated with GSK2578215A had IL-1 $\beta$ levels similar to that of Lrrk2 KO animals as well as increased bacterial burden in the spleen, blood, and peritoneal fluid. In vitro, they also demonstrated that, like Lrrk2 KO cells, wild-type macrophages treated with Lrrk2 kinase inhibitors also had decreased NLRC4 inflammasome activation (Liu et al., 2017). These data contradicts part of our own because we did not find any differences in bacterial burden in Lrrk2 kinase-dead mice. This could be due to genetic vs. pharmacological ablation of the kinase activity, or due to non-specific (i.e., off-target) effects by the inhibitor used by Liu et al. (2017). Importantly (given the implications for human trials), further experiments using genetic and pharmacological Lrrk2 kinase inhibition in conjunction will be critical to fully determine the effects of these inhibitors.

I have done some preliminary work with pharmacological inhibition of Lrrk2 kinase in vitro using BMDMs from wild-type mice either treated or untreated with a potent, selective Lrrk2 kinase inhibitor, MLi-2. When measuring cell survival and viral burden in cells following reovirus T3D infection, we did not find any differences between treated and untreated cells in either of these readouts. This supports our in vivo data that suggests Lrrk2 kinase is not required for this protein's role in immune response. Further work is being done utilizing the MLi-2 inhibitor in vivo.

We next investigated these kinase-dead mutant mice in our reovirus T3D paradigm. I originally hypothesized that these Lrrk2 ${ }^{\text {D1994S }}$ mice would have worsened survival in response to reovirus T3D infection, but in fact found the complete opposite. Lrrk2 kinase-dead mice had significantly increased survival compared to wild-type mice, with heterozygous mice falling somewhere in the middle. Indeed, Lrrk $2^{\mathrm{D} 1994 \mathrm{~S}}$ homozygous mice had a survival rate of $\sim 50 \%$, whereas Lrrk $2^{\text {D1994S }}$ heterozygous mice had $\sim 20 \%$ survival rate, and wild-type mice had only 
$\sim 10 \%$ survival rate (Fig. 3.4). This suggests that inhibiting the kinase activity in this disease context exerts a protective phenotype.

This was unexpected and is being further investigate in vivo and in vitro to explain this interesting phenomenon. Preliminary in vitro data using bone marrow-derived macrophages from wild-type and Lrrk $2^{\text {D1994S }}$ mice suggests immune signaling is decreased in kinase-dead cells, as indicated by pSTAT1/tSTAT1 protein abundance (Fig. 3.13). Kinase-dead BMDMs had decreased activated STAT1 abundance following reovirus infection compared to wild-type BMDMs (though not statistically significant). This may be indicative of decreased cytokine signaling in kinase-dead mice, which results in a protective phenotype in vivo; however, further validation is needed to support this hypothesis. Next, flow cytometry will be employed to measure the abundance of infiltrating myeloid cells, immunohistochemistry to measure microglial activation, and ELISA measurements of cytokine and chemokine protein levels in infected organs.

In our in vivo paradigm, we next measured viral burden in wild-type, heterozygous, and Lrrk2 ${ }^{\text {D1994S }}$ mice. Strikingly, we observed a difference in viral burden in the lung at an early time point of disease, $3 \mathrm{dpi}$, whereby the Lrrk2 ${ }^{\mathrm{D} 1994 \mathrm{~S}}$ kinase-dead mice had a significantly higher viral burden than wild-type mice. Wild-type mice had a mean viral titre of $1.99 \times 10^{6} \mathrm{PFU} / \mathrm{g}$ in the lung at 3dpi whereas Lrrk2 ${ }^{\mathrm{D} 1994 \mathrm{~S}}$ mice had significantly higher mean viral titre of $1.80 \times 10^{7} \mathrm{PFU} / \mathrm{g}$. Of note, as shown by LRRK2 mRNA studies and Lrrk2 Western blotting, the gene's expression level is the highest in mammalian lungs (Shutinoski et al. 2019). This difference was no longer discernable at the later stage of disease, in either the lungs or the brain (Fig. 3.5). We suggest that the lack of Lrrk2 kinase activity is affecting viral clearance at the early initial stages of disease that is recovered later in disease. Furthermore, it is possible that the lack of Lrrk2 kinase is causing a 
decreased inflammatory response, which affects viral clearance early in disease, but proves beneficial with respect to negative health outcomes (i.e., survival).

A seemingly unrelated recent paper in the field of virology helps to support this hypothesis: Chen et al. (2019) investigated the role of a new anti-inflammatory cytokine inhibitor in influenzaA pathogenesis and found that administration time of the drug plays an important role. They found that administering the drug to influenza-A infected mice one or two days after mice were infected did not rescue the mice or give any benefit, as measured by survival rate. However, when the drug was administered three or four days after the infection, the mice were fully recovered, going from $\sim 20 \%$ survival to $100 \%$ (Chen et al., 2019). These data suggest that an inflammatory response is important at the early stages of disease, but is less important and possibly harmful in later stages of disease. If we are correct in our idea that Lrrk2 kinase (and especially hyperactive kinase) causes a pro-inflammatory response, this notion supports our data that mice lacking Lrrk2 kinase cannot control the viral replication early in disease, but eventually recovers later in disease, and this lack of inflammation actually provides a survival benefit later in disease.

Though we have focused mainly on peripheral readouts, other reports have indicated that wild-type Lrrk2 pharmacological inhibition leads to decreased immune signaling of microglia as well. Moehle et al. (2012) have demonstrated that microglia primary cultures that are treated with a Lrrk2 kinase inhibitor have decreased TNF $\alpha$ signaling and microglia skeletal remodeling following LPS infection compared to untreated wild-type microglia. This supports our findings that inhibiting Lrrk2 kinase leads to a reduced immune response, though our work is the first to suggest that this can provide an immune benefit in the context of a potentially lethal encephalitis. 


\section{3. p.G2019S: Gain-of-Function}

While investigating the role of wild-type Lrrk2 kinase, we uncovered another equally important understanding: the role of p.G2019S in immune response is a gain-of-function. To elaborate, this increased kinase activity is not simply increasing the basal, physiological function of LRRK2, but conferring entirely new functions and properties within immune response. We can conclude this for two reasons: one, the lack of Lrrk2 kinase did not affect the survival of the mouse in two infectious paradigms; and two: Lrrk2 knockout and Lrrk2 kinase-dead mice had minimal effect on viral burden following infection, whereas Lrrk2 ${ }^{\mathrm{G} 2019 \mathrm{~S}}$ consistently cleared infectious burden more efficiently than wild-type. Therefore, it would appear that Lrrk2 ${ }^{\mathrm{G} 2019 \mathrm{~S}}$ mice have a function that is novel to the wild-type protein, likely in the inflammatory immune response and initiation. Taken together with the other hypotheses of this data set, our results suggest that the Lrrk $2^{\mathrm{G} 2019 \mathrm{~S}}$ mutation is causing a harmful effect, i.e., in the response to a viral pathogen that has invaded the brain.

There is a great body of evidence demonstrating that LRRK2 kinase inhibitors may be efficient in reversing any adverse effects associated with the p.G2019S mutation. For example, Howlett et al. (2017) have found that the p.G2019S mutation was associated with increased mitochondrial DNA (mtDNA) damage, in both Lrrk2 $2^{\mathrm{G} 2019 \mathrm{~S}}$ rats and in PD patient-derived lymphoblastoma cell lines. They did not find mtDNA damage in kinase-dead or wild-type animals. Most strikingly, they found that treating PD patient-derived cells with a LRRK2 kinase inhibitor prevented or restored this mtDNA damage (Howlett et al., 2017).

Secondly, Moehle et al. (2012), also demonstrated that the increased binding they observed in p.G2019S expressing cells between Lrrk2 ${ }^{\mathrm{G} 2019 \mathrm{~S}}$ and actin-regulatory proteins was decreased when these cells were pretreated with Lrrk2 kinase inhibitors. Therefore, this could be reversed by 
treatment with Lrrk2 kinase inhibitors, potentially reversing some of the effects of the myeloid cell recruitment and pro-inflammatory profile associated with the p.G2019S mutation (Moehle et al., 2012).

However, one must consider recent work from the lab of Dr. Andy West. Researchers investigated the response of the p.G2019S mutant to two common Lrrk2 kinase inhibitors, MLi-2 and PF360, in rats (Kelly et al., 2018). Using Lrrk2 ${ }^{\mathrm{G} 2019 \mathrm{~S}}$ overexpressing rats treated with either of the inhibitors via their chow diet, they demonstrated that Lrrk2 ${ }^{\mathrm{G} 2019 \mathrm{~S}}$ rats are much more resilient to both inhibitors (though specifically MLi-2) than wild-type rats, as measured via pSer935 levels as a ratio over total Lrrk2. Interestingly, this was seen specifically in the brain rather than the lungs. The lungs had similar inhibition of Lrrk2 kinase in both wild-type and Lrrk2 ${ }^{\mathrm{G} 2019 \mathrm{~S}}$ rats, whereas the brains of Lrrk2 ${ }^{\mathrm{G} 2019 \mathrm{~S}}$ rats were highly resilient, still showing a great deal of Lrrk2 specific phosphorylation, even at the highest concentrations of inhibition. Similarly, the Lrrk2 ${ }^{\mathrm{G} 2019 \mathrm{~S}}$ rats did not show the same phenotypes that wild-type mice did following inhibition, including lysosomal defects and discoloration in the kidney (Kelly et al., 2018). This suggests that Lrrk2 ${ }^{\mathrm{G} 2019 \mathrm{~S}}$ resiliency to kinase inhibition and kinase inhibitor-induced pathology could be supported by a constitutively active, gain-of-function effect.

\subsection{LRRK2 Acts in a Pathogen- and Organ- Dependent Manner}

Interestingly, we observed differential effects of the three Lrrk2 genotypes (Lrrk2 ${ }^{\mathrm{G} 2019 \mathrm{~S}}$, knock-out, Lrrk2 ${ }^{\mathrm{D} 1994 \mathrm{~S}}$ ) in response to the different pathogens. Specifically, the Lrrk2 knockout mice had decreased survival following viral infection and no change following a bacterial infection; Lrrk2 ${ }^{\mathrm{G} 2019 \mathrm{~S}}$ had decreased survival following viral and increased survival following bacterial; and lastly, Lrrk2 ${ }^{\text {D1994S }}$ had increased survival following viral and no change following a 
bacterial infection. Therefore, not only did variants respond differently from each other in each paradigm, but the same variants responded differently to each paradigm. There are two possible explanations as to how Lrrk2 may respond differently to distinct infection paradigms, although not mutually exclusive: that Lrrk2 is behaving in a pathogen-dependent and an organ-dependent manner. These conclusions are supported by a multitude of previously published works.

Firstly, Härtlova et al. (2018) has demonstrated, that in response to a Mycobacterium tuberculosis bacterial infection, Lrrk2 knockout and Lrrk2 kinase-inhibited macrophages showed better control of the bacteria. Interestingly, Lrrk2 knockout mice had enhanced immune response and pathogen clearance in vivo in response to the same pathogen; however, this control over bacterial burden was only seen at the early stage of disease (14dpi) and no longer seen in the later stages (56dpi). In addition, $\operatorname{Lrrk}^{\mathrm{G} 2019 \mathrm{~S}}$ derived macrophages had worsened control of the Mycobacterium pathogen and decreased signal of lysosomal markers (Härtlova et al., 2018).

Secondly, my second research team (located at Carleton University) has investigated the role of paraquat toxicity and Lrrk2 KO mice, p.G2019S over-expressing mice, and wild-type mice (Rudyk et al., 2019). Paraquat was administered via six injections over a period of time, and sickness scores, inflammation state (measured by IL-6 levels), corticosterone levels, and other measurements were taken. Interestingly, Lrrk2 $\mathrm{KO}$ mice were protected from all paraquat toxicity; they had lower sickness scores compared to wild-type mice, and lower IL-6 and corticosterone levels than wild-type littermates. Of note, the p.G2019S-transgenic (rather than knock-in used in the study here) mice did not differ from wild-type mice following paraquat injection in any regard except corticosterone - suggesting the p.G2019S mutation may be involved in the HPA axis (Rudyk et al., 2019). 
Lastly, Daher et al. (2014) have demonstrated that Lrrk2 KO rats were protected against dopaminergic neurodegeneration that was seen in wild-type rats following intracerebral LPS injections. They also demonstrated that Lrrk2 $\mathrm{KO}$ rats did not elicit dopaminergic cell loss following transduction of human $\alpha$-synuclein as the wild-type rats did following the same treatment (Daher et al., 2014).

In each of these studies, when using a distinct bacterial pathogen, a viral mimic, or neurotoxin, the Lrrk2 knockout mice were protected against the noxious agent. These studies attribute the protective phenotype of the Lrrk2 KO to a decreased inflammatory response (Härtlova et al., 2018; Rudyk et al., 2019; Daher et al., 2014) and in some cases (Daher et al., 2014) to decreased myeloid cell recruitment, which is a consistent finding in the field.

The direct implications of these studies contradict somewhat the results we have found with the Lrrk2 KO mice; however, altogether the common conclusion does not change: the Lrrk2 protein is highly implicated in inflammatory response, and the lack of Lrrk2 leads to a decreased inflammation. It is the contextual framework that is important and helps explain the phenotypic result, i.e., is this beneficial or harmful in response to infection? Importantly, in our paradigm, we employ live, replication-competent microbes, which is different from most studies (though not all: i.e., Härtlova et al., 2018).

Taken together, these suggest that the Lrrk2 protein responds to infectious threats in a complex, pathogen-dependent manner; hence, great earnest must be taking by the field in order to demonstrate all the ways in which Lrrk2 responds to different pathogens.

There is also recent evidence in the literature to support the notion that Lrrk2 is working in an organ-dependent manner. In our studies alone, Lrrk2, and its kinase activity, seems to respond to pathogens differently in the periphery $v s$. the brain, and in a manner dependent on where the 
pathogen originates (see Section 4.6). Lrrk2 is differentially expressed throughout the body, and may in part, account for the organ-specific response of Lrrk2. For example, Lrrk2 is much more highly expressed in the brain than the periphery, and more in immune cells than neurons (Hakimi et al., 2011). Specifically, LRRK2 in the brain is expressed more highly in glial cells, especially activated microglia, than in neurons. In the periphery, specifically the kidney, LRRK2 is expressed mostly in collecting duct cells and important in toxin clearance (Boddu et al., 2015) and in the lungs, LRRK2 is most highly expressed in alveolar cells, which are believed to be important at the interface of environmental pathogens, creating the surfactant in the lung (Herzig et al., 2011).

Similarly, mutations or inhibition of Lrrk2 kinase has different effects in different organs. Herzig et al. (2011) found that, at 6 weeks of age, Lrrk2 KO mice have cellular pathology in the lung and kidneys, whereas Lrrk2 kinase-dead mice only have cellular pathology in the kidneys. These data suggest that both the Lrrk2 protein and its kinase activity are important in an organspecific function of Lrrk2. Intriguingly, Lrrk2 ${ }^{\mathrm{G} 2019 \mathrm{~S}}$ mice did not have kidney or lung pathology, and were similar to wild-type mice (Herzig et al., 2011). Fuji et al. (2015) also identified that when using two separate LRRK2 kinase inhibitors in non-human primates, there is an increase in abnormal cytoplasmic accumulation of secretory lamellar bodies in type II pneumocytes, though this was not seen in the kidney.

Therefore, there is vast body of work to suggest that LRRK2 is serving an organ-specific role in the body. LRRK2 is expressed at different levels throughout different tissues, and seems to be playing a unique role throughout the body as well. The evidence from us and others supports the idea that LRRK2 has an organ-specific function, in the immune system and otherwise. 


\subsection{LRRK2 Female Sex Bias}

Of great interest, we observed a stark female sex bias with Lrrk2 variants in response to infection. Namely, that the Lrrk $2^{\mathrm{G} 2019 \mathrm{~S}}$ mutation affects females primarily (if not only) (Shutinoski et al., 2019); and the heterozygous mice of Lrrk2 knockout (Shutinoski et al., 2019) and of Lrrk2 kinase-dead colonies behave in a sex-determined manner, whereby female carriers behave the same as the homozygous mutant, and the male carrier behave the same as the homozygous wildtypes. This a very interesting finding corresponding to human data that there is a female sex bias with LRRK2-associated PD, despite the male sex bias with PD in general (reviewed in: Schlossmacher et al., 2017). Secondly, females have an earlier age of onset and higher penetrance associated with the p.G2019S mutation in humans (Goldworm et al., 2005). If females are more susceptible to mutations in the LRRK2 allele, and a microbial pathogen/dysregulated immune response are linked to disease etiology, it could explain why more female LRRK2 mutation carriers develop PD than males. Considering how low the penetrance rate is with Lrrk $2^{\mathrm{G} 2019 \mathrm{~S}}$ mutations, following our original 'two hit' theory, it is possible that females are genetically more susceptible and therefore infectious agents and other environmental 'triggers' have better chance of 'initiating' PD. This remains theoretical, but the sex difference identified in mice is definitely an interesting insight into the workings of LRRK2-associated PD, considering the same bias is seen in humans.

This intriguing sex bias may be explained by multiple reasons proposed in the literature. First, and most obviously, would be the influence of sex hormones. Females have a high abundance of estradiol, whereas males have higher abundance of androgens. These hormones influence multiple aspects of immune function: estrogen receptors respond differently to microbial metabolites than males (Park and Choi, 2017); estrogen promotes immune regulation via endosomal TLRs and TLR8 while males favour TLR2 and TLR4 regulation (Marriott et al., 2006); 
and interactions between sex hormones and environmental factors, such as smoking (interestingly linked as a protective factor for PD), cause the two sexes to react variably (Schlossmacher et al., 2017; Sugiyama et al., 2010). Interestingly, reports have also noted differential effects of the two sex hormones on microbiota composition. This is of great interest to the PD field, especially regarding the Braak staging hypothesis (2002) and the growing interest in microbiota interactions in disease pathogenesis (for example: Heintz-Buschart et al., 2018). One may argue that the mice employed in our viral infection paradigm may not be affected by sex hormones as they are infected as early as post-natal day 1 (therefore pre-puberty); however, multiple reports have detected androgens and estradiol as early as p1 in rats and mice (Konkle and McCarthy, 2011; ToranAllerand et al., 2005; Bakker et al., 2006).

Secondly, in development, males and females have differential microglial patterns (regarding morphology and abundance) in different brain regions that continues throughout life (Schwarz et al., 2012). It appears that in specific regions such as the amygdala, male mice have more microglia than females, early in development at p4, whereas later in life at p30 and p60, females have more ramified and developed microglia in these regions than males (Schwarz et al., 2012). This ramified state of the microglia in females may contribute to a heightened immune response.

Another explanation may fall in the sex-specific genetic composition between males and females. Females carry two X-chromosomes, and one chromosome is inactivated. However, often inactivation is incomplete leading to overexpression of $\mathrm{X}$-linked genes and genetic mosaicism in many female cells (Quintero et al., 2012). Many immune genes are X-linked including NOX-2, $C D 40 L$, and cytokine components such as IL-1R activated kinase (IRAKI) and the IL-2R $\gamma$ chain (Rubtsov et al., 2010; Quintero et al., 2012). Secondly, 10\% of miRNAs are encoded on the X- 
chromosome, some of which are involved in immune signaling (Pinheiro et al., 2011). Therefore, females may be affected by differential X-chromosome regulation and inheritance patterns that males are not affected by. Indeed, in humans, females tend to have enhanced immune reactivity, which includes higher antibody development, sustained inflammation, and stronger response to viral infection than males (Gaillard and Spinedi, 1998). Males tend to have a larger response to acute infection, whereas females are more likely to provide a sustained, chronic response, which could explain the fact that females are more likely to develop autoimmune disorders (Ngo et al., 2014). Taken together, these data supports our hypothesis that LRRK2 $2^{\mathrm{G} 2019 \mathrm{~S}}$ is associated with a heightened, sustained inflammatory response, and that females could be more affected by this mutation as they are already predisposed to chronic inflammation.

\subsection{LRRK2 Immune Function: Brain vs. Periphery}

A great deal of current research in the field of LRRK2 biology has focused on its role in the central nervous system (for example: in synaptic function: Matikainen-Ankney et al., 2018; in dopaminergic neurodegeneration: Giaime et al., 2017). Building upon previous works and our own, we wished to investigate the role of LRRK 2 in the periphery compared to in the brain-specific immune response. Specifically, we were driven by the fact that LRRK2 is lowly expressed in the brain compared to the periphery (Hakimi et al., 2011) and the fact that our Lrrk2 variants had strong modulation on disease outcomes in a purely peripheral disease paradigm (Shutinoski et al., 2019).

We employed a paradigm in which we intracerebrally infected mice with reovirus T3D directly into the brains of pups at p1. Unexpectedly, considering the stark survival benefit seen in Lrrk2 ${ }^{\mathrm{D} 1994 \mathrm{~S}}$ mice infected nasally, we did not see any survival benefit or deficit in Lrrk2 ${ }^{\mathrm{D} 1994 \mathrm{~S}}$ mice 
compared to wild-type mice or heterozygous mice, as mice of each genotype began to die at 9dpi, and majority were dead by $12 \mathrm{dpi}$. In fact, all genotypes had almost identical time-to-deaths. When separating based on sexes, we still did not see any genotypic differences.

To account for the possibility that our paradigm was too lethal to identify any genotypic differences, we tested multiple variations to the paradigm. Through optimization, we chose our standard dose (and the dose used in Fig. 3.8) to be $5 \times 10^{2}$ PFU per mouse. We had also tested cohorts with 50 PFU per mouse and $5 \times 10^{3}$ PFU per mouse and did not see any differences on survival (data not shown). At a dose of 50 PFU and lower, the variability and stochastic effects between each mouse become too great to draw reliable conclusions. Secondly, though not done with any Lrrk2 mouse model, a separate scientist in parallel performed mock intracerebral injections using PBS in $\mathrm{C} 57 \mathrm{Bl} / 6 \mathrm{p} 1$ pups and found a $0 \%$ death rate, indicating that no mortality was occurring from the injection alone (Christopher Rousso, Earl Brown, Michael Schlossmacher, unpublished; data not shown).

Next, to test whether further development may prolong the time-to-death following intracerebral injection, I next employed a paradigm in which I infected mice at p3 instead of p1, to allow the pup brain to develop further as well as the immune system. Again, we found no genotypic differences in survival in both sexes combined or either of the sexes individually. Indeed, the time-to-death was nearly equivalent to that of the mice infected at p1 (i.e., majority of the mice succumbed at 9-12dpi).

We have yet to successfully prove that this paradigm is not too fatal to all genotypes to uncover a difference; however, previous literature would suggest that this paradigm is sufficient to identify genotypic differences in survival. Beckham et al. (2011) investigated the effect of caspase-3 deletion on survival and viral burden following viral infection. They used mice on the 
same background as we did (C57Bl/6), the same virus and strain (reovirus T3D), and the same dose of virus $\left(10^{2} \mathrm{PFU}\right)$. Indeed, they identified a stark survival benefit in caspase-3 full KO mice compared to caspase- $3^{+/-}$heterozygous mice (which also showed about $\sim 10 \%$ survival) (Beckham et al., 2011). Of note, we have seen $100 \%$ mortality in all mouse models we have tested with intracerebral reovirus infection. They did not test wild-types in survival, which should have $100 \%$ mortality (Beckham et al., 2011).

Based on these data from Beckham et al. (2011), this paradigm can be sensitive enough to identify survival differences between genotypes. Therefore, though still with caution, we may begin to draw conclusion from our data. Taken together, our data from the nasal and intracerebral paradigms suggest that Lrrk2 exerts its immune function predominantly in the periphery rather than the brain. Lrrk $2^{\text {D1994S }}$ provided a strong survival benefit in response to a systemic infection with secondary encephalitis, but did not influence survival when the virus was administered directly into the brain. These data suggests, that in the nasal, systemic paradigm, the peripheral Lrrk2 ${ }^{\mathrm{D} 1994 \mathrm{~S}}$ was engaging the pathogen (i.e., virus) more efficiently in the periphery before the virus reached the brain, and that the input of microglia and brain-specific Lrrk2 ${ }^{\mathrm{D} 1994 \mathrm{~S}}$ protein seemed to be minimal.

We next investigated a second Lrrk2 variant in this direct brain inoculation. Again, we found no differences in time-to-death between wild-type mice and Lrrk2 ${ }^{\mathrm{G} 2019 \mathrm{~S}}$ mice following an intracerebral injection (Fig. 3.11). Importantly, our lab only identified survival differences in the intranasal paradigm when looking at females alone - presently, our sample set for Lrrk2 ${ }^{\mathrm{G} 2019}$ intracerebrally infected mice is too small to separate based on sexes, therefore it is very possible a difference may be identified when we have a succinct cohort of both males and females carrying the p.G2019S mutations. However, at the moment, our data suggest that $\mathrm{Lrrk}^{\mathrm{G} 2019}$ also functions 
in the context of immune responses to a virulent pathogen mainly outside the nervous system.

Specifically, these data sets suggest that variants in Lrrk2 modulate immune response predominantly in the periphery over the brain. This is not to say Lrrk2 serves no function in the brain, but our data suggest allelic variants in Lrrk2 exert an effect on immune response in the periphery rather than the brain as they modulated survival outcomes in a systemic infection but not in a direct brain infection.

Our data help support the hypothesis that mutations in LRRK2 may not be directly affecting the health of DA neurons, but rather exerting the toxic effects directly on immune cells in the periphery, which in turn have secondary, deleterious effects on DA neurons of the SNpc. Kozina et al. (2018) published an elegant paper recently highlighting the role of peripheral immune components and Lrrk2 mutants in the degeneration of DA neurons. They utilized a p.R1441G mouse model and an LPS injection neuroinflammation model to demonstrate that Lrrk2 mutant mice had heightened brain neuroinflammatory profiles (as measured by cytokine expression) compared to wild-type mice. Indeed, p.R1441G knock-in mice had increased IFN- $\gamma$ levels in the brain as long as two months following the conclusion of LPS treatment. Interestingly, they established that these mice did not have increased myeloid cell recruitment to the brain and that although they had heightened activated microglia, these microglia were not producing a high abundance of cytokines. Therefore, the authors conclude that it is the infiltrating peripheral molecules (i.e., cytokines) that are driving the inflammatory profile in the brains of these mice (Kozina et al., 2018). This is stark evidence contributing to the hypothesis that DA neuronal death can be due, in part, to infiltrating immune components from the periphery to the brain, rather than brain-derived. Additionally, this is another case in which variants in Lrrk2 (in this case p.R1441G) are work predominantly in peripheral immune cells rather than brain-specific cells (i.e., microglia, 
neurons) (Kozina et al., 2018).

More support of peripheral cytokines affecting brain health has come from Pott Goddoy et al. (2008): IL-1 is capable of crossing the blood brain barrier, and it has been shown that chronic, systemic IL-1 administration can lead to DA neuronal death in mice. Similarly, human data has shown that SNpc samples from post-mortem human PD samples shows leukocyte extravasation as well as damage to the blood brain barrier (Kortekaas et al., 2005). The authors of a cleverly titled review posit that a subtle, cumulative increase in brain cytokine levels over time could be leading to eventual neurotoxicity to the SNpc of LRRK2 mutant carriers, which could be reflected by the late-onset of LRRK2-associated PD (Greggio et al., 2012).

Next, in our research, we investigated viral burden in the brains and livers of intracerebrally infected mice. We investigated an early time point of disease (3dpi) and a later time point (8dpi) to represent the peak of disease before mice begin to succumb to the illness. At 3dpi, we found no significant differences in viral burden in either organ, though the sample set is in the process of being increased to fully conclude if there is no difference.

At 8dpi, we were intrigued by two facts. One, the high viral burden in the livers of these animals and two, the significant difference in viral burden in the brains of these mice. To start, we measured viral burden in livers of intracerebrally infected mice to measure the spread from the brain to the periphery. The viral burdens in the liver at $8 \mathrm{dpi}$ were $\sim 10^{6} \mathrm{PFU} / \mathrm{g}$ which was relatively high for a virus with tropism for the brain, administered directly to the brain. However, when we examined H\&E stained IHC slides from these mice, we observed a large tissue wound from the needle tract (data not shown), therefore we concluded that the needle tract damage caused leakage to the blood brain barrier early in infection, allowing much of the virus to spread and replicate in the liver. 
Secondly, Lrrk2 ${ }^{\text {D1994s }}$ mice had $\sim 1$-fold lower PFU/g in the brain at 8 dpi than wild-type mice. We were surprised and confused by these findings and are continuing to pursue the explanation behind this result. Of note, majority of the wild-type viral burden samples were from wild-type $\mathrm{x}$ wild-type homozygous pairings. Therefore, before concluding on these data, we plan to add more wild-type samples from heterozygous pairings to account for litter variability. If these data holds true, it contradicts (but does not invalidate) our nasal data for Lrrk2 ${ }^{\mathrm{D} 1994 \mathrm{~S}}$ mice. However, this would continue to add to the notion that Lrrk2 and variants in this protein are exerting a function that is organ-dependent. We are further investigating this result, including measuring myeloid cell recruitment, cytokine expression levels in infected brains, and microglia activation to see if any of these components could be affecting viral clearance in Lrrk2 ${ }^{\mathrm{D} 1994 \mathrm{~S}}$ mice. Additionally, we wish to measure viral burden in brains and livers of infected Lrrk2 ${ }^{\mathrm{G} 2019 \mathrm{~S}}$ mice to identify if this variant has any effect on brain viral burden following this direct-brain inoculation.

An important limitation of our intracerebral inoculation paradigm is that our injections are not stereotactically delivered by the operator. Importantly, each and every injection was done by the same operator, therefore injections were as consistent as possible within each cohort. However, in future studies, we hope to employ a method that is consistent and particular to one brain region. Our next studies involve infecting adult mice via stereotactic injection with vesicular stomata virus (VSV) in each of the Lrrk2 variants to retest our brain $v$ s. periphery paradigm, as well as testing a viral infection in the context of adult mice.

\section{Conclusion}

We have consistently demonstrated a role for Lrrk 2 in the immune system. Allelic variants in Lrrk2 have the ability to modulate host immunity to multiple different pathogens; some variants providing protection and some conferring detriment. Specifically, I have provided novel evidence 
that suggest Lrrk2 is functioning in the immune system independent of its kinase activity in the wild-type state, and may in fact confer protection in the context of a viral paradigm. Secondly, I have shown that Lrrk2 ${ }^{\mathrm{D} 1994 \mathrm{~S}}$ kinase-dead mice behave in the immune system on a female sex bias, consistent with results from Lrrk2 $2^{\mathrm{G} 2019 \mathrm{~S}}$ and Lrrk2 KO mice. Through this work with the kinasedead mutant, I have also confirmed that the p.G2019S mutation confers a gain-of-function, and I have added to our growing body of evidence that suggests that Lrrk2 works in an organ- and pathogen- dependent manner. Lastly, taking two important paradigms together, nasal inoculation and direct-brain inoculation of reovirus T3D in Lrrk2 ${ }^{\mathrm{D} 1994 \mathrm{~S}}$ and Lrrk2 ${ }^{\mathrm{G} 2019 \mathrm{~S}}$ p1 pups, I have produced results that suggest Lrrk2's role in the immune system is exerted predominantly in the periphery rather than the brain.

There is great momentum with this body of work that must be pursued further. Namely to (i) validate the periphery vs. brain findings of Lrrk2's immune function in an adult model using direct-brain VSV inoculation of adult mice in all Lrrk2 variants (Lrrk2 ${ }^{\text {D1994S }}$, Lrrk2 ${ }^{\mathrm{G} 2019 \mathrm{~S}}$, Lrrk2 $\mathrm{KO}$ ); (ii) to investigate the effects of pharmacological Lrrk2 kinase inhibition (using MLi-2) in our multiple infection paradigms by inhibiting wild-type mice, Lrrk2 ${ }^{\mathrm{D} 1994 \mathrm{~S}}$ mice and Lrrk2 ${ }^{\mathrm{G} 2019 \mathrm{~S}}$ mice; and (iii) most importantly, to investigate the mechanisms behind these findings by employing in vitro primary macrophage cultures and ex vivo readouts on infected mouse tissue. The data in this thesis and the data that will come from future studies will have direct impacts on elucidating LRRK2 biology, explaining PD pathogenesis, and identifying cause-directed treatments for both heritable and idiopathic cases of Parkinson's disease. 


\section{References}

Alessi, D. R., \& Sammler, E. (2018). LRRK2 kinase in Parkinson's disease. Science, 360(6384), 36-37. https://doi.org/10.1126/science.aar5683

Anheim, M., Elbaz, A., Lesage, S., Durr, A., Condroyer, C., Viallet, F., ... Brice, A. (2012). Penetrance of Parkinson disease in glucocerebrosidase gene mutation carriers. Neurology, 78(6), 417-420. https://doi.org/10.1212/WNL.0b013e318245f476

Arai, H., Furuya, T., Mizuno, Y., \& Mochizuki, H. (2006). Inflammation and infection in Parkinson's disease. Histology and Histopathology, 21(6), 673-678. https://doi.org/10.14670/HH-21.673

Baba, Y., Higuchi, M., Abe, H., Fukuyama, K., Onozawa, R., Uehara, Y., ... Yamada, T. (2012). Anti-cholinergics for axial symptoms in Parkinson's disease after subthalamic stimulation. Clinical Neurology and Neurosurgery, 114(10), 1308-1311. https://doi.org/10.1016/j.clineuro.2012.03.046

Bakker, J., De Mees, C., Douhard, Q., Balthazart, J., Gabant, P., Szpirer, J., \& Szpirer, C. (2006). Alpha-fetoprotein protects the developing female mouse brain from masculinization and defeminization by estrogens. Nature Neuroscience, 9(2), 220-226. https://doi.org/10.1038/nn1624

Beckham, J. D., Tuttle, K. D., \& Tyler, K. L. (2010). Caspase-3 activation is required for reovirus-induced encephalitis in vivo. Journal of Neurovirology, 16(4), 306-317. https://doi.org/10.3109/13550284.2010.499890

Bembi, B., Zambito Marsala, S., Sidransky, E., Ciana, G., Carrozzi, M., Zorzon, M., ... Capus, L. (2003). Gaucher's disease with Parkinson's disease: Clinical and pathological aspects. Neurology, 61(1), 99-101. https://doi.org/10.1212/01.WNL.0000072482.70963.D7

Blanchard, V., Raisman-Vozari, R., Vyas, S., Michel, P. P., Javoy-Agid, F., Uhl, G., \& Agid, Y. (1994). Differential expression of tyrosine hydroxylase and membrane dopamine transporter genes in subpopulations of dopaminergic neurons of the rat mesencephalon. Brain Research. Molecular Brain Research, 22(1-4), 29-38. Retrieved from http://www.ncbi.nlm.nih.gov/pubmed/7912404

Boddu, R., Hull, T. D., Bolisetty, S., Hu, X., Moehle, M. S., Daher, J. P. L., ... West, A. B. (2015). Leucine-rich repeat kinase 2 deficiency is protective in rhabdomyolysis-induced kidney injury. Human Molecular Genetics, 24(14), 4078-4093. https://doi.org/10.1093/hmg/ddv147

Bohnen, N. I., Albm, R. L., Koeppe, R. A., Wernette, K. A., Kilbourn, M. R., Minoshima, S., \& Frey, K. A. (2006). Positron Emission Tomography of Monoaminergic Vesicular Binding in Aging and Parkinson Disease. Journal of Cerebral Blood Flow \& Metabolism, 26(9), 1198 1212. https://doi.org/10.1038/sj.jcbfm.9600276

Bonifati, V. (2003). Mutations in the DJ-1 Gene Associated with Autosomal Recessive EarlyOnset Parkinsonism. Science, 299(5604), 256-259. https://doi.org/10.1126/science.1077209

Bové, J., \& Perier, C. (2012). Neurotoxin-based models of Parkinson's disease. Neuroscience, 211, 51-76. https://doi.org/10.1016/j.neuroscience.2011.10.057

Braak, H., Tredici, K. Del, Rüb, U., de Vos, R. A. ., Jansen Steur, E. N. ., \& Braak, E. (2003). Staging of brain pathology related to sporadic Parkinson's disease. Neurobiology of Aging, 24(2), 197-211. https://doi.org/10.1016/S0197-4580(02)00065-9 
Brady, R. O., Kanfer, J. N., \& Shapiro, D. (1965). Metabolism of glucocerebrosides. II. Evidence of an enzymatic deficiency in Gaucher's Disease. Biochemical and Biophysical Research Communications, 18, 221-225.

Brockmann, K., Srulijes, K., Hauser, A.-K., Schulte, C., Csoti, I., Gasser, T., \& Berg, D. (2011). GBA-associated PD presents with nonmotor characteristics. Neurology, 77(3), 276-280. https://doi.org/10.1212/WNL.0b013e318225ab77

Burns, R. S., Markey, S. P., Phillips, J. M., \& Chiueh, C. C. (1984). The neurotoxicity of 1methyl-4-phenyl-1,2,3,6-tetrahydropyridine in the monkey and man. The Canadian Journal of Neurological Sciences. Le Journal Canadien Des Sciences Neurologiques, 11(1 Suppl), 166-168. Retrieved from http://www.ncbi.nlm.nih.gov/pubmed/6608980

Chartier-Harlin, M.-C., Kachergus, J., Roumier, C., Mouroux, V., Douay, X., Lincoln, S., ... Destée, A. (2004). $\alpha$-synuclein locus duplication as a cause of familial Parkinson's disease. The Lancet, 364(9440), 1167-1169. https://doi.org/10.1016/S0140-6736(04)17103-1

Chen, S., Liu, G., Chen, J., Hu, A., Zhang, L., Sun, W., ... Chen, X. (2019). Ponatinib Protects Mice From Lethal Influenza Infection by Suppressing Cytokine Storm. Frontiers in Immunology, 10(June). https://doi.org/10.3389/fimmu.2019.01393

Chen, W., Zhang, X., \& Huang, W.-J. (2016). Role of neuroinflammation in neurodegenerative diseases (Review). Molecular Medicine Reports, 13(4), 3391-3396. https://doi.org/10.3892/mmr.2016.4948

Cook, D. A., Kannarkat, G. T., Cintron, A. F., Butkovich, L. M., Fraser, K. B., Chang, J., ... Tansey, M. G. (2017). LRRK2 levels in immune cells are increased in Parkinson's disease. Npj Parkinson's Disease, 3(1), 1-11. https://doi.org/10.1038/s41531-017-0010-8

Daher, J. P. L., Volpicelli-Daley, L. A., Blackburn, J. P., Moehle, M. S., \& West, A. B. (2014). Abrogation of -synuclein-mediated dopaminergic neurodegeneration in LRRK2-deficient rats. Proceedings of the National Academy of Sciences, 111(25), 9289-9294. https://doi.org/10.1073/pnas.1403215111

Deleidi, M., \& Isacson, O. (2012). Viral and Inflammatory Triggers of Neurodegenerative Diseases. Science Translational Medicine, 4(121), 121ps3-121ps3. https://doi.org/10.1126/scitranslmed.3003492

Denali Therapeutics Inc. (2018). Denali Therapeutics Announces Positive Clinical Results From LRRK2 Inhibitor Program for Parkinson's Disease. Retrieved from: http://investors.denalitherapeutics.com/news-releases/news-release-details/denalitherapeutics-announces-positive-clinical-results-lrrk2\#ir-pages

Dimitrova, N., Zamudio, J. R., Jong, R. M., Soukup, D., Resnick, R., Sarma, K., ... Jacks, T. (2017). Public Access NIH Public Access. PLoS ONE, 32(7), 736-740. https://doi.org/10.1371/journal.pone.0178059

Doty, R. L., Deems, D. A., \& Stellar, S. (1988). Olfactory dysfunction in parkinsonism: A general deficit unrelated to neurologic signs, disease stage, or disease duration. Neurology, 38(8), 1237-1237. https://doi.org/10.1212/WNL.38.8.1237

Drokhlyansky, E., Göz Aytürk, D., Soh, T. K., Chrenek, R., O’Loughlin, E., Madore, C., ... Cepko, C. L. (2017). The brain parenchyma has a type I interferon response that can limit virus spread. Proceedings of the National Academy of Sciences, 114(1), E95-E104. https://doi.org/10.1073/pnas.1618157114

Dzamko, N. L. (2012). Leucine-rich repeat kinase 2 (LRRK2). Science-Business EXchange, 5(22), 579-579. https://doi.org/10.1038/scibx.2012.579 
Dzamko, N., Deak, M., Hentati, F., Reith, A. D., Prescott, A. R., Alessi, D. R., \& Nichols, R. J. (2010). Inhibition of LRRK2 kinase activity leads to dephosphorylation of Ser 910 /Ser 935 , disruption of 14-3-3 binding and altered cytoplasmic localization. Biochemical Journal, 430(3), 405-413. https://doi.org/10.1042/BJ20100784

Dzamko, N., Inesta-Vaquera, F., Zhang, J., Xie, C., Cai, H., Arthur, S., ... Alessi, D. R. (2012). The IkappaB Kinase Family Phosphorylates the Parkinson's Disease Kinase LRRK2 at Ser935 and Ser910 during Toll-Like Receptor Signaling. PLoS ONE, 7(6), e39132. https://doi.org/10.1371/journal.pone.0039132

Dzamko, N., Rowe, D. B., \& Halliday, G. M. (2016). Increased peripheral inflammation in asymptomatic leucine-rich repeat kinase 2 mutation carriers. Movement Disorders, 31(6), 889-897. https://doi.org/10.1002/mds.26529

Eguchi, T., Kuwahara, T., Sakurai, M., Komori, T., Fujimoto, T., Ito, G., ... Iwatsubo, T. (2018). LRRK2 and its substrate Rab GTPases are sequentially targeted onto stressed lysosomes and maintain their homeostasis. Proceedings of the National Academy of Sciences, 115(39), E9115-E9124. https://doi.org/10.1073/pnas.1812196115

Fan, Y., Howden, A. J. M., Sarhan, A. R., Lis, P., Ito, G., Martinez, T. N., ... Sammler, E. M. (2018). Interrogating Parkinson's disease LRRK2 kinase pathway activity by assessing Rab10 phosphorylation in human neutrophils. Biochemical Journal, 475(1), 23-44. https://doi.org/10.1042/BCJ20170803

Fava, V. M., Manry, J., Cobat, A., Orlova, M., Van Thuc, N., Ba, N. N., ... Schurr, E. (2016). A Missense LRRK2 Variant Is a Risk Factor for Excessive Inflammatory Responses in Leprosy. PLOS Neglected Tropical Diseases, 10(2), e0004412. https://doi.org/10.1371/journal.pntd.0004412

Fell, M. J., Mirescu, C., Basu, K., Cheewatrakoolpong, B., DeMong, D. E., Ellis, J. M., ... Morrow, J. A. (2015). MLi-2, a Potent, Selective, and Centrally Active Compound for Exploring the Therapeutic Potential and Safety of LRRK2 Kinase Inhibition. Journal of Pharmacology and Experimental Therapeutics, 355(3), 397-409. https://doi.org/10.1124/jpet.115.227587

Ferrer, I. (2011). Neuropathology and Neurochemistry of Nonmotor Symptoms in Parkinson's Disease. Parkinson's Disease, 2011(708404), 1-13. https://doi.org/10.4061/2011/708404

Franchi, L., Amer, A., Body-Malapel, M., Kanneganti, T.-D., Özören, N., Jagirdar, R., ... Núñez, G. (2006). Cytosolic flagellin requires Ipaf for activation of caspase- 1 and interleukin $1 \beta$ in salmonella-infected macrophages. Nature Immunology, 7(6), 576-582. https://doi.org/10.1038/ni1346

Fuji, R. N., Flagella, M., Baca, M., S. Baptista, M. A., Brodbeck, J., Chan, B. K., .. Watts, R. J. (2015). Effect of selective LRRK2 kinase inhibition on nonhuman primate lung. Science Translational Medicine, 7(273), 273ra15-273ra15. https://doi.org/10.1126/scitranslmed.aaa3634

Gaillard, R. C., \& Spinedi, E. (1998). Sex- and stress-steroids interactions and the immune system: evidence for a neuroendocrine-immunological sexual dimorphism. Domestic Animal Endocrinology, 15(5), 345-352. Retrieved from http://www.ncbi.nlm.nih.gov/pubmed/9785038

Gardet, A., Benita, Y., Li, C., Sands, B. E., Ballester, I., Stevens, C., ... Podolsky, D. K. (2010). LRRK2 Is Involved in the IFN- $\gamma$ Response and Host Response to Pathogens. The Journal of Immunology, 185(9), 5577-5585. https://doi.org/10.4049/jimmunol.1000548 
Gasser, T. (2009). Mendelian forms of Parkinson's disease. Biochimica et Biophysica Acta (BBA) - Molecular Basis of Disease, 1792(7), 587-596. https://doi.org/10.1016/j.bbadis.2008.12.007

Gauvin, L., Bennett, S., Liu, H., Hakimi, M., Schlossmacher, M., Majithia, J., \& Brown, E. G. (2013). Respiratory infection of mice with mammalian reoviruses causes systemic infection with age and strain dependent pneumonia and encephalitis. Virology Journal, 10(1), 67. https://doi.org/10.1186/1743-422X-10-67

Giaime, E., Tong, Y., Wagner, L. K., Yuan, Y., Huang, G., \& Shen, J. (2017). Age-Dependent Dopaminergic Neurodegeneration and Impairment of the Autophagy-Lysosomal Pathway in LRRK-Deficient Mice. Neuron, 96(4), 796-807.e6. https://doi.org/10.1016/j.neuron.2017.09.036

Goedert, M., Spillantini, M. G., Del Tredici, K., \& Braak, H. (2013). 100 years of Lewy pathology. Nature Reviews Neurology, 9(1), 13-24.

https://doi.org/10.1038/nrneurol.2012.242

Goldwurm, S. (2005). The G6055A (G2019S) mutation in LRRK2 is frequent in both early and late onset Parkinson's disease and originates from a common ancestor. Journal of Medical Genetics, 42(11), e65-e65. https://doi.org/10.1136/jmg.2005.035568

Greffard, S., Verny, M., Bonnet, A.-M., Beinis, J.-Y., Gallinari, C., Meaume, S., ... Duyckaerts, C. (2006). Motor Score of the Unified Parkinson Disease Rating Scale as a Good Predictor of Lewy Body-Associated Neuronal Loss in the Substantia Nigra. Archives of Neurology, 63(4), 584. https://doi.org/10.1001/archneur.63.4.584

Greggio, E., Civiero, L., Bisaglia, M., \& Bubacco, L. (2012). Parkinson's disease and immune system: Is the culprit LRRKing in the periphery? Journal of Neuroinflammation, 9(1), 1. https://doi.org/10.1186/1742-2094-9-94

Hakimi, M., Selvanantham, T., Swinton, E., Padmore, R. F., Tong, Y., Kabbach, G., ... Schlossmacher, M. G. (2011). Parkinson's disease-linked LRRK2 is expressed in circulating and tissue immune cells and upregulated following recognition of microbial structures. Journal of Neural Transmission, 118(5), 795-808.

https://doi.org/10.1007/s00702-011-0653-2

Hanamsagar, R., \& Bilbo, S. D. (2016). Sex differences in neurodevelopmental and neurodegenerative disorders: Focus on microglial function and neuroinflammation during development. The Journal of Steroid Biochemistry and Molecular Biology, 160, 127-133. https://doi.org/10.1016/j.jsbmb.2015.09.039

Härtlova, A., Herbst, S., Peltier, J., Rodgers, A., Bilkei-Gorzo, O., Fearns, A., ... Gutierrez, M. G. (2018). LRRK2 is a negative regulator of Mycobacterium tuberculosis phagosome maturation in macrophages . The EMBO Journal, 37(12), e98694. https://doi.org/10.15252/embj.201798694

Hawkes, C. H., Del Tredici, K., \& Braak, H. (2007). Parkinson's disease: a dual-hit hypothesis. Neuropathology and Applied Neurobiology, 33(6), 599-614. https://doi.org/10.1111/j.13652990.2007.00874.x

Hawkes, C. H., Del Tredici, K., \& Braak, H. (2009). Parkinson's Disease. Annals of the New York Academy of Sciences, 1170(1), 615-622. https://doi.org/10.1111/j.17496632.2009.04365.x

Healy, D. G., Abou-Sleiman, P. M., \& Wood, N. W. (2004). PINK, PANK, or PARK? A clinicians' guide to familial parkinsonism. The Lancet Neurology, 3(11), 652-662. https://doi.org/10.1016/S1474-4422(04)00905-6 
Healy, D. G., Falchi, M., O’Sullivan, S. S., Bonifati, V., Durr, A., Bressman, S., ... Wood, N. W. (2008). Phenotype, genotype, and worldwide genetic penetrance of LRRK2-associated Parkinson's disease: a case-control study. The Lancet Neurology, 7(7), 583-590. https://doi.org/10.1016/S1474-4422(08)70117-0

Heintz-Buschart, A., Pandey, U., Wicke, T., Sixel-Döring, F., Janzen, A., Sittig-Wiegand, E., ... Wilmes, P. (2018). The nasal and gut microbiome in Parkinson's disease and idiopathic rapid eye movement sleep behavior disorder. Movement Disorders, 33(1), 88-98. https://doi.org/10.1002/mds.27105

Herbst, S., \& Gutierrez, M. G. (2019). LRRK2 in Infection: Friend or Foe? ACS Infectious Diseases, 5(6), 809-815. https://doi.org/10.1021/acsinfecdis.9b00051

Herzig, M. C., Kolly, C., Persohn, E., Theil, D., Schweizer, T., Hafner, T., ... Shimshek, D. R. (2011). LRRK2 protein levels are determined by kinase function and are crucial for kidney and lung homeostasis in mice. Human Molecular Genetics, 20(21), 4209-4223. https://doi.org/10.1093/hmg/ddr348

Hickman, S., Izzy, S., Sen, P., Morsett, L., \& El Khoury, J. (2018). Microglia in neurodegeneration. Nature Neuroscience, 21(10), 1359-1369. https://doi.org/10.1038/s41593-018-0242-x

Ho, F. Y., Rosenbusch, K. E., \& Kortholt, A. (2014). The Potential of Targeting LRRK2 in Parkinson's Disease. In A Synopsis of Parkinson's Disease. https://doi.org/10.5772/57362

Howlett, E. H., Jensen, N., Belmonte, F., Zafar, F., Hu, X., Kluss, J., ... Sanders, L. H. (2017). LRRK2 G2019S-induced mitochondrial DNA damage is LRRK2 kinase dependent and inhibition restores mtDNA integrity in Parkinson's disease. Human Molecular Genetics, 26(22), 4340-4351. https://doi.org/10.1093/hmg/ddx320

Hui, K. Y., Fernandez-Hernandez, H., Hu, J., Schaffner, A., Pankratz, N., Hsu, N.-Y., ... Peter, I. (2018). Functional variants in the LRRK2 gene confer shared effects on risk for Crohn's disease and Parkinson's disease. Science Translational Medicine, 10(423), eaai7795. https://doi.org/10.1126/scitranslmed.aai7795

Imamura, K., Hishikawa, N., Sawada, M., Nagatsu, T., Yoshida, M., \& Hashizume, Y. (2003). Distribution of major histocompatibility complex class II-positive microglia and cytokine profile of Parkinson's disease brains. Acta Neuropathologica, 106(6), 518-526. https://doi.org/10.1007/s00401-003-0766-2

Jang, H., Boltz, D., Sturm-Ramirez, K., Shepherd, K. R., Jiao, Y., Webster, R., \& Smeyne, R. J. (2009). Highly pathogenic H5N1 influenza virus can enter the central nervous system and induce neuroinflammation and neurodegeneration. Proceedings of the National Academy of Sciences, 106(33), 14063-14068. https://doi.org/10.1073/pnas.0900096106

Johansen, K. K., Torp, S. H., Farrer, M. J., Gustavsson, E. K., \& Aasly, J. O. (2018). A Case of Parkinson's Disease with No Lewy Body Pathology due to a Homozygous Exon Deletion in Parkin. Case Reports in Neurological Medicine, 2018, 1-4. https://doi.org/10.1155/2018/6838965

Jordan, E. O. (1927). Epidemic Influenza. A Survey. Chicago: American Medical Association. Kachergus, J., Mata, I. F., Hulihan, M., Taylor, J. P., Lincoln, S., Aasly, J., ... Toft, M. (2005). Identification of a Novel LRRK2 Mutation Linked to Autosomal Dominant Parkinsonism: Evidence of a Common Founder across European Populations. The American Journal of Human Genetics, 76(4), 672-680. https://doi.org/10.1086/429256

Kalia, L. V, \& Lang, A. E. (2015). Parkinson's disease. The Lancet, 386(9996), 896-912. https://doi.org/10.1016/S0140-6736(14)61393-3 
Kang, U.-B., \& Marto, J. A. (2017). Leucine-rich repeat kinase 2 and Parkinson's disease. Proteomics, 17(1-2), 1600092. https://doi.org/10.1002/pmic.201600092

Kawakami, F., Yabata, T., Ohta, E., Maekawa, T., Shimada, N., Suzuki, M., ... Obata, F. (2012). LRRK2 Phosphorylates Tubulin-Associated Tau but Not the Free Molecule: LRRK2Mediated Regulation of the Tau-Tubulin Association and Neurite Outgrowth. PLoS ONE, 7(1), e30834. https://doi.org/10.1371/journal.pone.0030834

Kelly, K., Wang, S., Boddu, R., Liu, Z., Moukha-Chafiq, O., Augelli-Szafran, C., \& West, A. B. (2018). The G2019S mutation in LRRK2 imparts resiliency to kinase inhibition. Experimental Neurology, 309, 1-13. https://doi.org/10.1016/j.expneurol.2018.07.012

Kett, L. R., Boassa, D., Ho, C. C.-Y., Rideout, H. J., Hu, J., Terada, M., ... Dauer, W. T. (2012). LRRK2 Parkinson disease mutations enhance its microtubule association. Human Molecular Genetics, 21(4), 890-899. https://doi.org/10.1093/hmg/ddr526

Kim, K. S., Marcogliese, P. C., Yang, J., Callaghan, S. M., Resende, V., Abdel-Messih, E., ... Park, D. S. (2018). Regulation of myeloid cell phagocytosis by LRRK2 via WAVE2 complex stabilization is altered in Parkinson's disease. Proceedings of the National Academy of Sciences, 115(22), E5164-E5173. https://doi.org/10.1073/pnas.1718946115

Kitada, T., Asakawa, S., Hattori, N., Matsumine, H., Yamamura, Y., Minoshima, S., ... Shimizu, N. (1998). Mutations in the parkin gene cause autosomal recessive juvenile parkinsonism. Nature, 392(6676), 605-608. https://doi.org/10.1038/33416

Klein, C., \& Schlossmacher, M. G. (2007). Parkinson disease, 10 years after its genetic revolution: Multiple clues to a complex disorder. Neurology, 69(22), 2093-2104. https://doi.org/10.1212/01.wnl.0000271880.27321.a7

Konkle, A. T. M., \& McCarthy, M. M. (2011). Developmental Time Course of Estradiol, Testosterone, and Dihydrotestosterone Levels in Discrete Regions of Male and Female Rat Brain. Endocrinology, 152(1), 223-235. https://doi.org/10.1210/en.2010-0607

Kortekaas, R., Leenders, K. L., van Oostrom, J. C. H., Vaalburg, W., Bart, J., Willemsen, A. T. M., \& Hendrikse, N. H. (2005). Blood-brain barrier dysfunction in parkinsonian midbrain in vivo. Annals of Neurology, 57(2), 176-179. https://doi.org/10.1002/ana.20369

Kozina, E., Sadasivan, S., Jiao, Y., Dou, Y., Ma, Z., Tan, H., ... Smeyne, R. J. (2018). Mutant LRRK2 mediates peripheral and central immune responses leading to neurodegeneration in vivo. Brain, 141(6), 1753-1769. https://doi.org/10.1093/brain/awy077

Landreth, K. S. (2002). Critical window in the development of the rodent immune system. Human and Experimental Toxicology, 21, 493-498.

Langston, J., Ballard, P., Tetrud, J., \& Irwin, I. (1983). Chronic Parkinsonism in humans due to a product of meperidine-analog synthesis. Science, 219(4587), 979-980. https://doi.org/10.1126/science.6823561

Lee, H., James, W. S., \& Cowley, S. A. (2017). LRRK2 in peripheral and central nervous system innate immunity: its link to Parkinson's disease. Biochemical Society Transactions, 45(1), 131-139. https://doi.org/10.1042/bst20160262

Lesage, S., Patin, E., Condroyer, C., Leutenegger, A. L., Lohmann, E., Giladi, N., ... Brice, A. (2010). Parkinson's disease-related LRRK2 G2019S mutation results from independent mutational events in humans. Human Molecular Genetics, 19(10), 1998-2004. https://doi.org/10.1093/hmg/ddq081

LeWitt, P. A. (2008). Levodopa for the Treatment of Parkinson's Disease. New England Journal of Medicine, 359(23), 2468-2476. https://doi.org/10.1056/NEJMct0800326 
Liu, W., Liu, X., Li, Y., Zhao, J., Liu, Z., Hu, Z., ... Kang, Z. (2017). LRRK2 promotes the activation of NLRC4 inflammasome during Salmonella Typhimurium infection . The Journal of Experimental Medicine, 214(10), 3051-3066. https://doi.org/10.1084/jem.20170014

Lo, K. C., Geddes, J. F., Daniels, R. S., \& Oxford, J. S. (2003). Lack of detection of influenza genes in archived formalin-fixed, paraffin wax-embedded brain samples of encephalitis lethargica patients from 1916 to 1920. Virchows Archiv: An International Journal of Pathology, 442(6), 591-596. https://doi.org/10.1007/s00428-003-0795-1

MacLeod, D., Dowman, J., Hammond, R., Leete, T., Inoue, K., \& Abeliovich, A. (2006). The Familial Parkinsonism Gene LRRK2 Regulates Neurite Process Morphology. Neuron, 52(4), 587-593. https://doi.org/10.1016/j.neuron.2006.10.008

Marker, D. F., Puccini, J. M., Mockus, T. E., Barbieri, J., Lu, S. M., \& Gelbard, H. A. (2012). LRRK2 kinase inhibition prevents pathological microglial phagocytosis in response to HIV1 Tat protein. Journal of Neuroinflammation, 9(1), 261-273. https://doi.org/10.1186/17422094-9-261

Marriott, I., Bost, K. L., \& Huet-Hudson, Y. M. (2006). Sexual dimorphism in expression of receptors for bacterial lipopolysaccharides in murine macrophages: A possible mechanism for gender-based differences in endotoxic shock susceptibility. Journal of Reproductive Immunology, 71(1), 12-27. https://doi.org/10.1016/j.jri.2006.01.004

Martyn, C. N. (1997). Infection in childhood and neurological diseases in adult life. British Medical Bulletin, 53(1), 24-39.

Matikainen-Ankney, B. A., Kezunovic, N., Menard, C., Flanigan, M. E., Zhong, Y., Russo, S. J., ... Huntley, G. W. (2018). Parkinson's Disease-Linked LRRK2-G2019S Mutation Alters Synaptic Plasticity and Promotes Resilience to Chronic Social Stress in Young Adulthood. The Journal of Neuroscience, 38(45), 9700-9711. https://doi.org/10.1523/JNEUROSCI.1457-18.2018

Migdalska-Richards, A., \& Schapira, A. H. V. (2016). The relationship between glucocerebrosidase mutations and Parkinson disease. Journal of Neurochemistry, 139, 77 90. https://doi.org/10.1111/jnc.13385

Moehle, M. S., Webber, P. J., Tse, T., Sukar, N., Standaert, D. G., DeSilva, T. M., ... West, A. B. (2012). LRRK2 Inhibition Attenuates Microglial Inflammatory Responses. Journal of Neuroscience, 32(5), 1602-1611. https://doi.org/10.1523/JNEUROSCI.5601-11.2012

Moehle, M. S., Daher, J. P. L., Hull, T. D., Boddu, R., Abdelmotilib, H. A., Mobley, J., ... West, A. B. (2015). The G2019S LRRK2 mutation increases myeloid cell chemotactic responses and enhances LRRK2 binding to actin-regulatory proteins. Human Molecular Genetics, 24(15), 4250-4267. https://doi.org/10.1093/hmg/ddv157

Muñoz, E., Oliva, R., Obach, V., Martí, M. J., Pastor, P., Ballesta, F., \& Tolosa, E. (1997). Identification of Spanish familial Parkinson's disease and screening for the Ala53Thr mutation of the $\alpha$-synuclein gene in early onset patients. Neuroscience Letters, 235(1-2), 57-60. https://doi.org/10.1016/S0304-3940(97)00710-6

Nalls, M. A., Pankratz, N., Lill, C. M., Do, C. B., Hernandez, D. G., Saad, M., ... Singleton, A. B. (2014). Large-scale meta-analysis of genome-wide association data identifies six new risk loci for Parkinson's disease. Nature Genetics, 46(9), 989-993. https://doi.org/10.1038/ng.3043 
Ngo, S. T., Steyn, F. J., \& McCombe, P. A. (2014). Gender differences in autoimmune disease. Frontiers in Neuroendocrinology, 35(3), 347-369. https://doi.org/10.1016/j.yfrne.2014.04.004

Park, H.-J., \& Choi, J.-M. (2017). Sex-specific regulation of immune responses by PPARs. Experimental \& Molecular Medicine, 49(8), e364-e364. https://doi.org/10.1038/emm.2017.102

Parkinson, J. (2002). An Essay on the Shaking Palsy. The Journal of Neuropsychiatry and Clinical Neurosciences, 14(2), 223-236. https://doi.org/10.1176/jnp.14.2.223

Passini, M. A., \& Wolfe, J. H. (2001). Widespread Gene Delivery and Structure-Specific Patterns of Expression in the Brain after Intraventricular Injections of Neonatal Mice with an Adeno-Associated Virus Vector. Journal of Virology, 75(24), 12382-12392. https://doi.org/10.1128/JVI.75.24.12382-12392.2001

Patrick, K. L., Bell, S. L., Weindel, C. G., \& Watson, R. O. (2019). Exploring the "Multiple-Hit Hypothesis" of Neurodegenerative Disease: Bacterial Infection Comes Up to Bat. Frontiers in Cellular and Infection Microbiology, 9, 1-18. https://doi.org/10.3389/fcimb.2019.00138

Pinheiro, I., Dejager, L., \& Libert, C. (2011). X-chromosome-located microRNAs in immunity: Might they explain male/female differences? BioEssays, 33(11), 791-802. https://doi.org/10.1002/bies.201100047

Pott Godoy, M. C., Tarelli, R., Ferrari, C. C., Sarchi, M. I., \& Pitossi, F. J. (2008). Central and systemic IL-1 exacerbates neurodegeneration and motor symptoms in a model of Parkinson's disease. Brain, 131(7), 1880-1894. https://doi.org/10.1093/brain/awn101

Quintero, O. L., Amador-Patarroyo, M. J., Montoya-Ortiz, G., Rojas-Villarraga, A., \& Anaya, J.M. (2012). Autoimmune disease and gender: Plausible mechanisms for the female predominance of autoimmunity. Journal of Autoimmunity, 38(2-3), J109-J119. https://doi.org/10.1016/j.jaut.2011.10.003

Rauch, I., Müller, M., \& Decker, T. (2013). The regulation of inflammation by interferons and their STATs. JAK-STAT, 2(1), e23820. https://doi.org/10.4161/jkst.23820

Rodriguez-Oroz, M. C., Obeso, J. A., Lang, A. E., Houeto, J.-L., Pollak, P., Rehncrona, S., ... Van Blercom, N. (2005). Bilateral deep brain stimulation in Parkinson's disease: a multicentre study with 4 years follow-up. Brain, 128(10), 2240-2249. https://doi.org/10.1093/brain/awh571

Roosen, D. A., \& Cookson, M. R. (2016). LRRK2 at the interface of autophagosomes, endosomes and lysosomes. Molecular Neurodegeneration, 11(73), 1-10. https://doi.org/10.1186/s13024-016-0140-1

Rubtsov, A. V., Rubtsova, K., Kappler, J. W., \& Marrack, P. (2010). Genetic and hormonal factors in female-biased autoimmunity. Autoimmunity Reviews, 9(7), 494-498. https://doi.org/10.1016/j.autrev.2010.02.008

Rudenko, I. N., Kaganovich, A., Hauser, D. N., Beylina, A., Chia, R., Ding, J., ... Cookson, M. R. (2012). The G2385R variant of leucine-rich repeat kinase 2 associated with Parkinson's disease is a partial loss-of-function mutation. Biochemical Journal, 446(1), 99-111. https://doi.org/10.1042/BJ20120637

Rudyk, C., Dwyer, Z., \& Hayley, S. (2019). Leucine-rich repeat kinase-2 (LRRK2) modulates paraquat-induced inflammatory sickness and stress phenotype. Journal of Neuroinflammation, 16(1), 120. https://doi.org/10.1186/s12974-019-1483-7

Sanchez-Guajardo, V., Barnum, C. J., Tansey, M. G., \& Romero-Ramos, M. (2013). Neuroimmunological processes in Parkinson's disease and their relation to $\alpha$-synuclein: 
microglia as the referee between neuronal processes and peripheral immunity. ASN Neuro, 5(2), 113-139. https://doi.org/10.1042/AN20120066

Schapira, A. H., \& Jenner, P. (2011). Etiology and pathogenesis of Parkinson's disease. Movement Disorders, 26(6), 1049-1055. https://doi.org/10.1002/mds.23732

Schlossmacher, M. G., Tomlinson, J. J., Santos, G., Shutinoski, B., Brown, E. G., Manuel, D., \& Mestre, T. (2017). Modelling idiopathic Parkinson disease as a complex illness can inform incidence rate in healthy adults: the P R EDIGT score. European Journal of Neuroscience, 45(1), 175-191. https://doi.org/10.1111/ejn.13476

Schwarz, J. M., Sholar, P. W., \& Bilbo, S. D. (2012). Sex differences in microglial colonization of the developing rat brain. Journal of Neurochemistry, 948-63. https://doi.org/10.1111/j.1471-4159.2011.07630.x

Sheng, Z., Zhang, S., Bustos, D., Kleinheinz, T., Le Pichon, C. E., Dominguez, S. L., ... Zhu, H. (2012). Ser1292 Autophosphorylation Is an Indicator of LRRK2 Kinase Activity and Contributes to the Cellular Effects of PD Mutations. Science Translational Medicine, 4(164), 164ra161. https://doi.org/10.1126/scitranslmed.3004485

Shu, L., Zhang, Y., Sun, Q., Pan, H., \& Tang, B. (2019). A Comprehensive Analysis of Population Differences in LRRK2 Variant Distribution in Parkinson's Disease. Frontiers in Aging Neuroscience, 11. https://doi.org/10.3389/fnagi.2019.00013

Shulte, C., \& Gasser, T. (2011). Genetic basis of Parkinson's disease: inheritance, penetrance, and expression. The Application of Clinical Genetics, 67. https://doi.org/10.2147/TACG.S11639

Shutinoski, B., Alturki, N. A., Rijal, D., Bertin, J., Gough, P. J., Schlossmacher, M. G., \& Sad, S. (2016). K45A mutation of RIPK1 results in poor necroptosis and cytokine signaling in macrophages, which impacts inflammatory responses in vivo. Cell Death \& Differentiation, 23(10), 1628-1637. https://doi.org/10.1038/cdd.2016.51

Shutinoski, B., Hakimi, M., Harmsen, I. E., Lunn, M., Rocha, J., Lengacher, N., ... Schlossmacher, M. G. (in press - 2019). Lrrk2 Modulates Host Inflammation during Infections in a Pathogen-, Organ- and Sex-Dependent Manner. Science Translational Medicine.

Sian J, Youdim MBH, Riederer P, and G. M. (1999). MPTP-Induced Parkinsonian Syndromee (6th ed;; P. George J Siegel, MD, Editor-in-Chief, Bernard W Agranoff, MD, R Wayne Albers, PhD, Stephen K Fisher, PhD, and Michael D Uhler, Ed.). Phildadelphia: LippincottRaven.

Sidransky, E., Nalls, M. A., Aasly, J. O., Aharon-Peretz, J., Annesi, G., Barbosa, E. R., ... Ziegler, S. G. (2009). Multicenter Analysis of Glucocerebrosidase Mutations in Parkinson's Disease. New England Journal of Medicine, 361(17), 1651-1661. https://doi.org/10.1056/NEJMoa0901281

Skaper, S. D., Facci, L., Zusso, M., \& Giusti, P. (2018). An Inflammation-Centric View of Neurological Disease: Beyond the Neuron. Frontiers in Cellular Neuroscience, 12. https://doi.org/10.3389/fncel.2018.00072

Steger, M., Tonelli, F., Ito, G., Davies, P., Trost, M., Vetter, M., ... Mann, M. (2016). Phosphoproteomics reveals that Parkinson's disease kinase LRRK2 regulates a subset of Rab GTPases. ELife, 5. https://doi.org/10.7554/eLife.12813

Stoker, T. B., Torsney, K. M., \& Barker, R. A. (2018). Emerging Treatment Approaches for Parkinson's Disease. Frontiers in Neuroscience, 12, 693. https://doi.org/10.3389/fnins.2018.00693 
Sugiyama, D., Nishimura, K., Tamaki, K., Tsuji, G., Nakazawa, T., Morinobu, A., \& Kumagai, S. (2010). Impact of smoking as a risk factor for developing rheumatoid arthritis: a metaanalysis of observational studies. Annals of the Rheumatic Diseases, 69(01), 70-81. https://doi.org/10.1136/ard.2008.096487

Taneja, V. (2018). Sex Hormones Determine Immune Response. Frontiers in Immunology, 9, 15. https://doi.org/10.3389/fimmu.2018.01931

Tong, Y., Yamaguchi, H., Giaime, E., Boyle, S., Kopan, R., Kelleher, R. J., \& Shen, J. (2010). Loss of leucine-rich repeat kinase 2 causes impairment of protein degradation pathways, accumulation of -synuclein, and apoptotic cell death in aged mice. Proceedings of the National Academy of Sciences, 107(21), 9879-9884. https://doi.org/10.1073/pnas.1004676107

Toran-Allerand, C. D., Tinnikov, A. A., Singh, R. J., \& Nethrapalli, I. S. (2005). 17 $\alpha$-Estradiol: A Brain-Active Estrogen? Endocrinology, 146(9), 3843-3850. https://doi.org/10.1210/en.2004-1616

Tysnes, O.-B., \& Storstein, A. (2017). Epidemiology of Parkinson's disease. Journal of Neural Transmission, 124(8), 901-905. https://doi.org/10.1007/s00702-017-1686-y

Valente, E. M. (2004). Hereditary Early-Onset Parkinson's Disease Caused by Mutations in PINK1. Science, 304(5674), 1158-1160. https://doi.org/10.1126/science.1096284

West, A. B. (2017). Achieving neuroprotection with LRRK2 kinase inhibitors in Parkinson disease. Experimental Neurology, 298, 236-245. https://doi.org/10.1016/j.expneurol.2017.07.019

West, A. B., Moore, D. J., Choi, C., Andrabi, S. A., Li, X., Dikeman, D., ... Dawson, T. M. (2007). Parkinson's disease-associated mutations in LRRK2 link enhanced GTP-binding and kinase activities to neuronal toxicity. Human Molecular Genetics, 16(2), 223-232. https://doi.org/10.1093/hmg/ddl471

Woodward, K. (2017). Putting gender on the agenda. The Short Guide to Gender, 10, 1-20. https://doi.org/10.2307/j.ctt1t89g0g.5

Xiao, B., Goh, J.-Y., Xiao, L., Xian, H., Lim, K.-L., \& Liou, Y.-C. (2017). Reactive oxygen species trigger Parkin/PINK1 pathway-dependent mitophagy by inducing mitochondrial recruitment of Parkin. Journal of Biological Chemistry, 292(40), 16697-16708. https://doi.org/10.1074/jbc.M117.787739

Yan, R., \& Liu, Z. (2017). LRRK2 enhances Nod1/2-mediated inflammatory cytokine production by promoting Rip2 phosphorylation. Protein and Cell, 8(1), 55-66. https://doi.org/10.1007/s13238-016-0326-X

Yanguas-Casas, N. (2017). SM Gr up Neurological Disorders and Stroke Sex Di ff erences in Neurodegenerative. SMGroup, 3(1), 1-6. Retrieved from http://www.ncbi.nlm.nih.gov/entrez/query.fcgi? $\mathrm{cmd}=$ Retrieve \&db=PubMed\&dopt=Citation \&list_uids $=19507495$

Yu, L., Hu, F., Zou, X., Jiang, Y., Liu, Y., He, X., ... Xu, Y. (2009). LRRK2 R1628P contributes to Parkinson's disease susceptibility in Chinese Han populations from mainland China. Brain Research, 1296, 113-116. https://doi.org/10.1016/j.brainres.2009.08.047

Zhang, Q.-S., Heng, Y., Yuan, Y.-H., \& Chen, N.-H. (2017). Pathological $\alpha$-synuclein exacerbates the progression of Parkinson's disease through microglial activation. Toxicology Letters, 265, 30-37. https://doi.org/10.1016/j.toxlet.2016.11.002 
Zimprich, A., Biskup, S., Leitner, P., Lichtner, P., Farrer, M., Lincoln, S., ... Gasser, T. (2004). Mutations in LRRK2 Cause Autosomal-Dominant Parkinsonism with Pleomorphic Pathology. Neuron, 44(4), 601-607. https://doi.org/10.1016/j.neuron.2004.11.005

Post scriptum: A portion of this body of work contributed to a scientific journal article which can be found at:

Shutinoski, B., Hakimi, M., Harmsen, I. E., Lunn, M., Rocha, J., Lengacher, N., ... Schlossmacher, M. G. (in press - September 2019). Lrrk2 Modulates Host Inflammation during Infections in a Pathogen-, Organ- and Sex-Dependent Manner. Science Translational Medicine. 